Supporting Information for

\title{
Synthesis of a Tris(phosphaalkene)phosphine Ligand and Fundamental Organometallic Reactions on Its Sterically Shielded Metal Complexes
}

Preston M. Miura-Akagi, ${ }^{1}$ Mika L. Nakashige,${ }^{1}$ Caitlin K. Maile, ${ }^{1}$ Shelly M. Oshiro, ${ }^{1}$ Joshua R. Gurr, ${ }^{1}$ Wesley Y. Yoshida, ${ }^{1}$ A. Timothy Royappa, ${ }^{3}$ Colleen E. Krause, ${ }^{2}$ Arnold L. Rheingold, ${ }^{3}$ Russell P. Hughes, ${ }^{4}$ and Matthew F. Cain ${ }^{1}$

${ }^{1}$ Department of Chemistry, University of Hawai'i at Mānoa, 2545 McCarthy Mall, Honolulu, Hawaii 96822

2 Department of Chemistry, University of Hartford, 200 Bloomfield Avenue, West Hartford, Connecticut 06117

${ }^{3}$ Department of Chemistry, University of California, San Diego, 9500 Gilman Drive, La Jolla, California 92093

${ }^{4} 6128$ Burke Laboratory, Department of Chemistry, Dartmouth College, Hanover, New Hampshire 03755

Contact: mfcain@hawaii.edu

1. General Experimental Details

2. Experimental Details for the Synthesis of New Compounds (including NMR spectra and references)

3. DFT Computational Details

4. Electrochemistry Experimental Details including additional Cyclic Voltammetry Plots

5. Experimental Details for Crystal Structure Refinement and Acquisition (including references) 
General Experimental Details All manipulations were conducted under a nitrogen or argon atmosphere in a MBRAUN drybox or using Schlenk techniques unless otherwise specified. All glassware was oven-dried prior to use. Anhydrous grade acetonitrile, pentane, toluene, benzene, ether, dichloromethane, and THF were purchased from commercial suppliers (Aldrich or Acros) and pumped directly into the glove box. All solvents were stored over oven-activated $4 \AA$ molecular sieves (Aldrich). The following starting materials and reagents: $\left[\mathrm{RhCl}\left(\mathrm{C}_{2} \mathrm{H}_{4}\right)_{2}\right]_{2},\left[\mathrm{IrCl}(\mathrm{COE})_{2}\right]_{2}, \mathrm{AgOTf}, \mathrm{NaN}_{3}$ (and $\mathrm{Na}^{15} \mathrm{~N}_{3}$ ), and $\mathrm{Zn}$ dust were purchased from commercial suppliers. $\mathrm{PMe}_{3}$ was purchased from Strem and dried over molecular sieves prior to use. $\mathrm{Mes}^{*} \mathrm{PCl}_{2}$ and tris(aldehyde) B were prepared according to literature protocols. ${ }^{1}$ NMR spectra were obtained on Varian spectrometers operating at either $300 \mathrm{MHz}$ or $500 \mathrm{MHz}$; all spectra shown in the Supporting Information are on the $500 \mathrm{MHz}$ spectrometer. NMR chemical shifts are reported as ppm relative to tetramethylsilane, and are referenced to the residual proton or ${ }^{13} \mathrm{C}$ signal of the solvent $\left({ }^{1} \mathrm{H} \mathrm{CDCl}_{3}: 7.27 \mathrm{ppm} ;{ }^{1} \mathrm{H} \mathrm{C}_{6} \mathrm{D}_{6}: 7.16 \mathrm{ppm} ;{ }^{13} \mathrm{C} \mathrm{CDCl}_{3}\right.$ : $\left.77.16 \mathrm{ppm} ;{ }^{13} \mathrm{C} \mathrm{C}_{6} \mathrm{D}_{6}, 128.06 \mathrm{ppm}\right)$. Mass spectrometry was conducted at the University of California at Irvine (Dr. J. Greaves) on compound 1. Other mass spectroscopic data was obtained at the University of Hawai'i i at Mānoa on an Agilent 6545 Accurate-Mass Q-TOF LC/MS (NSF CHE-1532310). Analytical data were obtained from the CENTC Elemental Analysis Facility at the University of Rochester, funded by NSF CHE0650456. All X-ray quality crystals were analyzed at the Small Molecule X-ray Crystallography Facility located at the University of California at San Diego.

1 Cowley, A.H.; Norman, N.C.; Pakulski, M. Inorg. Synth. 1990, 27, 235-240. Whitnall, M.R.; Hii, K.K.; Pett-Thornton, M.; Kee, T.P. J. Organomet. Chem. 1997, 529, 35-50. 


\section{Experimental Details for the Synthesis of New Compounds Synthesis of tris(phosphaalkene)phosphine 1}

$\mathrm{Mes}^{*} \mathrm{PCl}_{2}$ (100 mg, $0.288 \mathrm{mmol}$ ) and $\mathrm{Zn}$ dust (94 mg, $1.44 \mathrm{mmol}, 5$ equiv) were combined in a vial and $2 \mathrm{~mL}$ of benzene was added. The heterogeneous mixture was cooled in the glove box freezer $\left(-35^{\circ} \mathrm{C}\right)$ for $15 \mathrm{~min}$. Treatment of the frozen benzene mixture with a room temperature solution of $\mathrm{PMe}_{3}(56 \mathrm{mg}, 0.736 \mathrm{mmol}, 2.6$ equiv) in $3 \mathrm{~mL}$ of benzene resulted in an olive green reaction mixture. The reaction mixture was stirred for 2-2.5 $\mathrm{h}$ at room temperature and filtered through a Celite plug. The bright yellow-orange filtrate (Mes*P=PMe ${ }_{3},{ }^{31} \mathrm{P} \mathrm{NMR}\left(\mathrm{C}_{6} \mathrm{H}_{6}\right): \delta 4.2$ and $134.3, J=580 \mathrm{~Hz}$ ) was added to solid tris(aldehyde) $\mathbf{B}(20 \mathrm{mg}, 0.058 \mathrm{mmol}, 1 / 5$ equiv) affording a homogeneous bright yellow solution. The solution was transferred to a J-Young tube, sealed, brought outside of the glove box, and heated to $60{ }^{\circ} \mathrm{C}$ for $16 \mathrm{~h}$. ${ }^{31} \mathrm{P}$ NMR spectroscopy confirmed complete conversion to the desired product. Identifiable byproducts included unreacted $\mathrm{Mes}^{*} \mathrm{P}=\mathrm{PMe}_{3}, \mathrm{PMe}_{3}(\mathrm{O})(\delta 31)$, $\mathrm{PMe}_{3}(\delta-62)$, and $\mathrm{PH}_{2} \mathrm{Mes}^{*}(\delta-130)$. The contents of the J-Young tube were then transferred to a vial (and exposed to oxygen) and concentrated under vacuum. The yellow residue was dissolved in $3 \mathrm{~mL}$ of pentane and filtered through a Celite plug. The filtrate was concentrated under vacuum and recrystallized from $5 \mathrm{~mL}$ of a 50:50 solution of THF and acetonitrile at $-20{ }^{\circ} \mathrm{C}$ to afford a yellow crystalline solid (50 mg, $0.044 \mathrm{mmol}, 76 \%$ ). Crystals suitable for X-ray diffraction were obtained by dissolving $25 \mathrm{mg}$ of the ligand in $4 \mathrm{~mL}$ of a 50:50 solution of THF and acetonitrile at $0{ }^{\circ} \mathrm{C}$. On scale-up to $1 \mathrm{~g}$ of $\mathrm{Mes}^{*} \mathrm{PCl}_{2}, 467 \mathrm{mg}$ of 1 was isolated (79\% yield); $1 / 5.5$ equiv of $\mathbf{B}$ is employed.

Elemental analysis data was consistently low (3 attempts) in carbon, for example: Anal. Calcd. for $\mathrm{C}_{75} \mathrm{H}_{102} \mathrm{P}_{4}$ : C, 79.89; $\mathrm{H}, 9.12$. Found: $\mathrm{C}, 79.33 ; \mathrm{H}, 9.30$. HRMS $\mathrm{m} / z$ calcd for $\mathrm{C}_{75} \mathrm{H}_{102} \mathrm{P}_{4} \mathrm{Na}(\mathrm{M}+\mathrm{Na})^{+}:$1149.6830. Found: 1149.6853 . ${ }^{31} \mathrm{P}\left\{{ }^{1} \mathrm{H}\right\} \mathrm{NMR}$ $\left(\mathrm{CD}_{2} \mathrm{Cl}_{2}\right): \delta 266.5$ (d, $J=30 \mathrm{~Hz}, 3 \mathrm{P}, \mathrm{P}=\mathrm{C}$ ), -32.3 (quartet, $J=30 \mathrm{~Hz}, 1 \mathrm{P}, \mathrm{PAr}_{3}$ ). ${ }^{1} \mathrm{H} \mathrm{NMR}$ $\left(\mathrm{CD}_{2} \mathrm{Cl}_{2}\right): \delta 8.57(\mathrm{dd}, J=25,5.5 \mathrm{~Hz}, 3 \mathrm{H}$, Mes*P=CHAr), $7.88(\mathrm{br} \mathrm{m}, 3 \mathrm{H}, \mathrm{Ar}), 7.30(\mathrm{t}, J=$ $7.5 \mathrm{~Hz}, 3 \mathrm{H}, \mathrm{Ar}$ ), 7.25 (6H, Mes*), 7.11 (t, $J=7.5 \mathrm{~Hz}, 3 \mathrm{H}, \mathrm{Ar}$ ), 6.55 (dd, $J=7.5,4.5 \mathrm{~Hz}$, $3 \mathrm{H}, \mathrm{Ar}$ ), 1.30 and 1.17 (overlapping singlets, 1 sharp and 1 broad, $81 \mathrm{H}, \mathrm{Mes}^{*}$ ). ${ }^{13} \mathrm{C}\left\{{ }^{1} \mathrm{H}\right\}$ NMR $\left(\mathrm{CD}_{2} \mathrm{Cl}_{2}\right): \delta 173.5(\mathrm{dd}, J=36,20.5 \mathrm{~Hz}, \mathrm{P}=\mathrm{C}), 154.2$ (Ar), 149.7 (Ar), 145.3 (dd, $J=25,14 \mathrm{~Hz}, \mathrm{Ar}), 139.8$ (d, $J=56 \mathrm{~Hz}, \mathrm{Ar}$ ), 135.1 (Ar), 134.8 (t, $J=11 \mathrm{~Hz}$, Ar), 129.3 (Ar), 128.3 (Ar), 127.1 (d, $J=27 \mathrm{~Hz}, \mathrm{Ar}), 121.9(\mathrm{Ar}), 38.2(\mathrm{CMe}), 35.2$ ( $t$ $\mathrm{Bu}), 33.7\left(\mathrm{CMe}_{3}\right), 31.5(t-\mathrm{Bu}) .{ }^{13} \mathrm{C} \mathrm{NMR}$ assignments were aided by DEPT experiments. 
${ }^{31} \mathrm{P}\left\{{ }^{1} \mathrm{H}\right\} \mathrm{NMR}\left(\mathrm{CD}_{2} \mathrm{Cl}_{2}\right)$

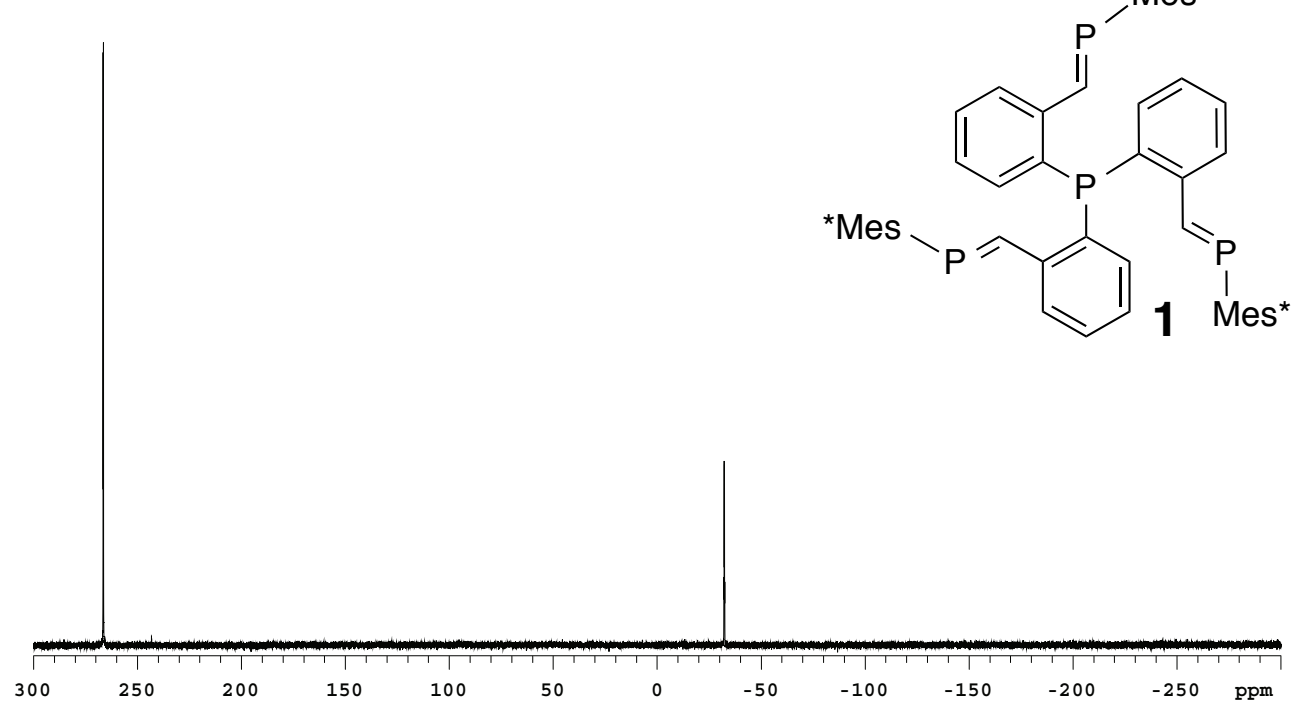

${ }^{31} \mathrm{P}\left\{{ }^{1} \mathrm{H}\right\} \mathrm{NMR}\left(\mathrm{CD}_{2} \mathrm{Cl}_{2}\right):$ Phosphaalkene signal $\left(J_{\mathrm{PP}}=30 \mathrm{~Hz}\right)$

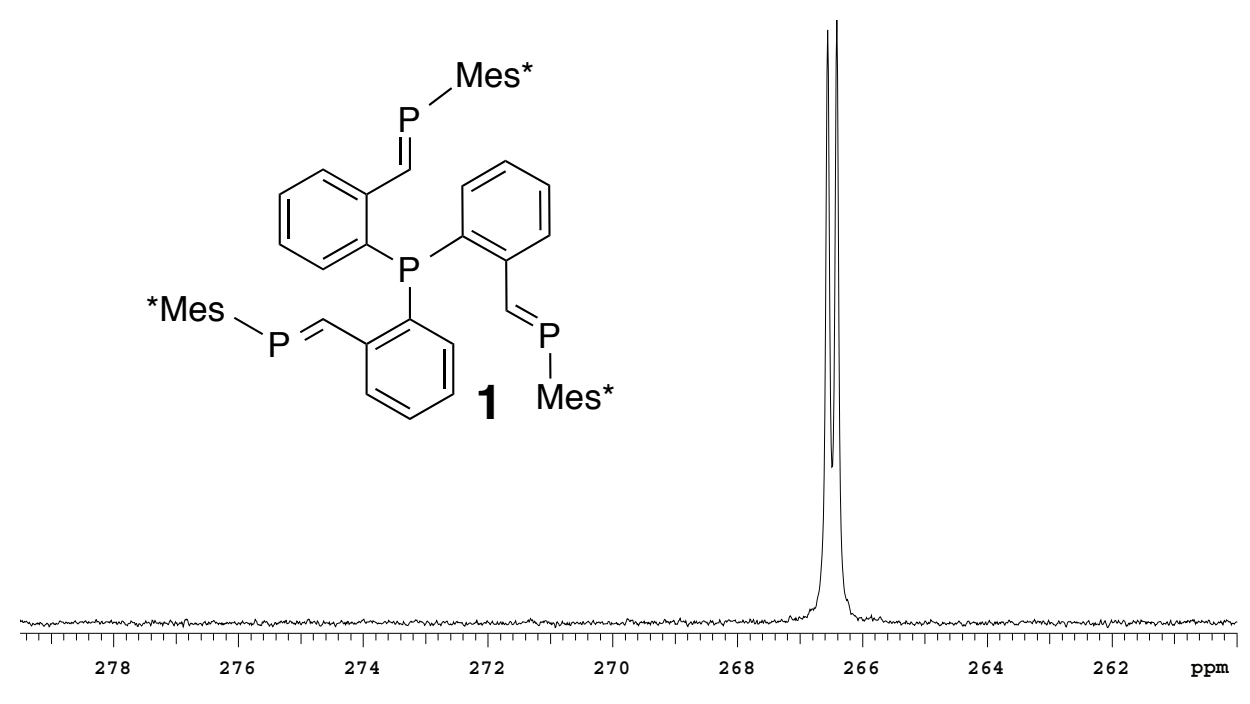


${ }^{31} \mathrm{P}\left\{{ }^{1} \mathrm{H}\right\}$ NMR $\left(\mathrm{CD}_{2} \mathrm{Cl}_{2}\right):$ Apical P $\left(\mathrm{JP}_{\mathrm{PP}}=30 \mathrm{~Hz}\right)$

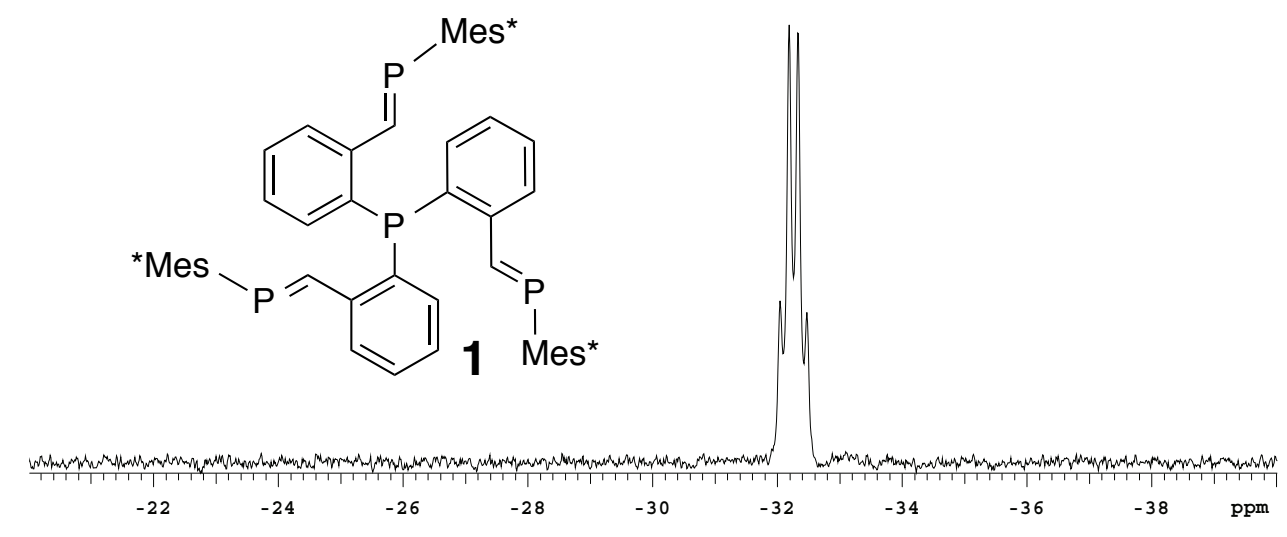

${ }^{1} \mathrm{H}$ NMR $\left(\mathrm{CD}_{2} \mathrm{Cl}_{2}\right)$ : Trace methanol and $\mathrm{H}_{2} \mathrm{O}$ present. The isolated solid is washed with methanol and placed under vacuum before being pumped into the glove box.<smiles>CP=Cc1ccccc1P(c1ccccc1C=PC)c1ccccc1CP=[P+](C)C</smiles>

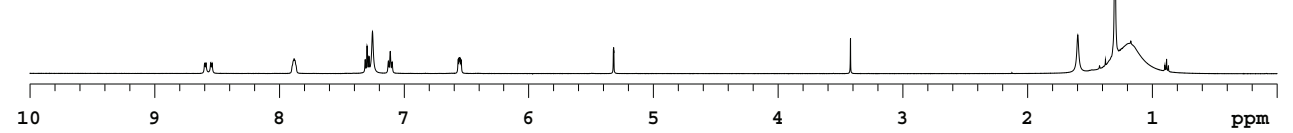


${ }^{1} \mathrm{H}$ NMR $\left(\mathrm{CD}_{2} \mathrm{Cl}_{2}\right)$ : Aryl and Phosphaalkene Region

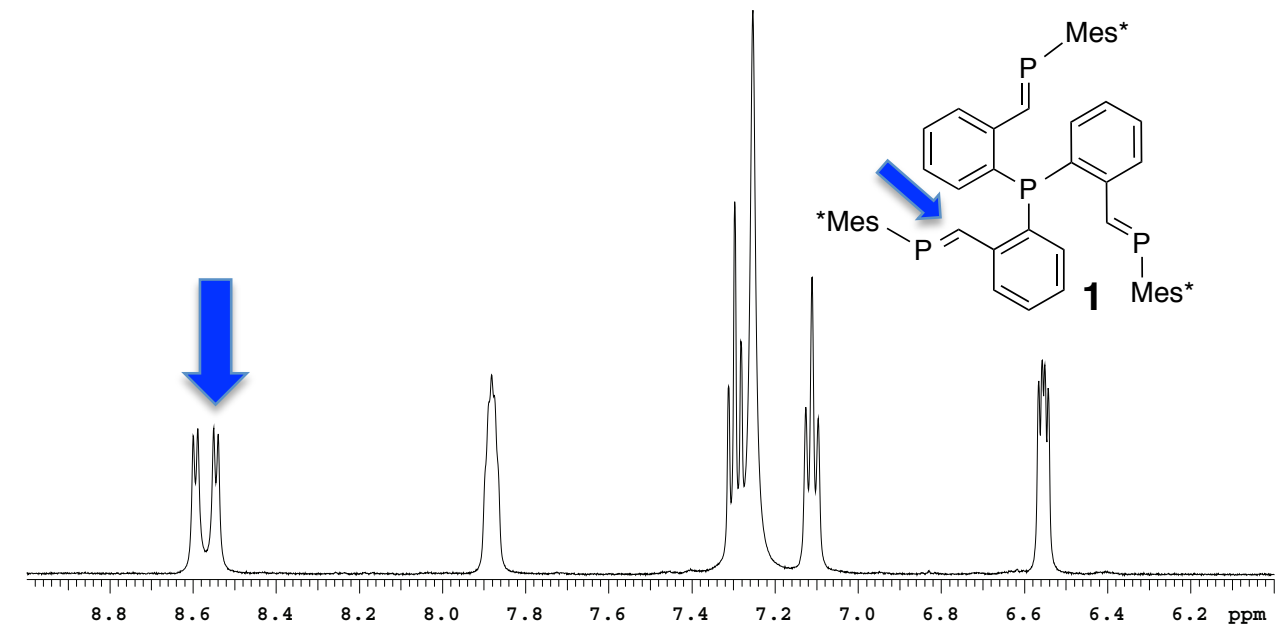

${ }^{13} \mathrm{C}\left\{{ }^{1} \mathrm{H}\right\}$ NMR $\left(\mathrm{CD}_{2} \mathrm{Cl}_{2}\right)$
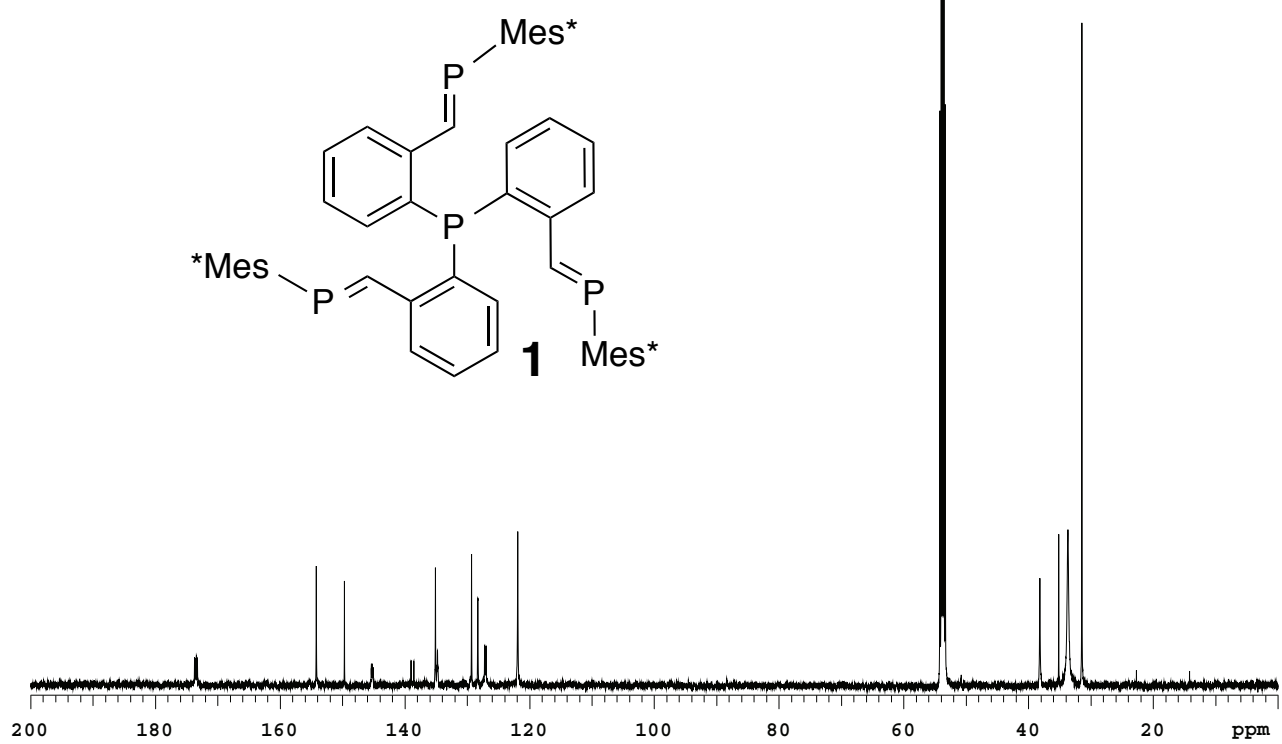
${ }^{13} \mathrm{C}\left\{{ }^{1} \mathrm{H}\right\}$ NMR $\left(\mathrm{CD}_{2} \mathrm{Cl}_{2}\right)$ : Aryl and Phosphaalkene Region

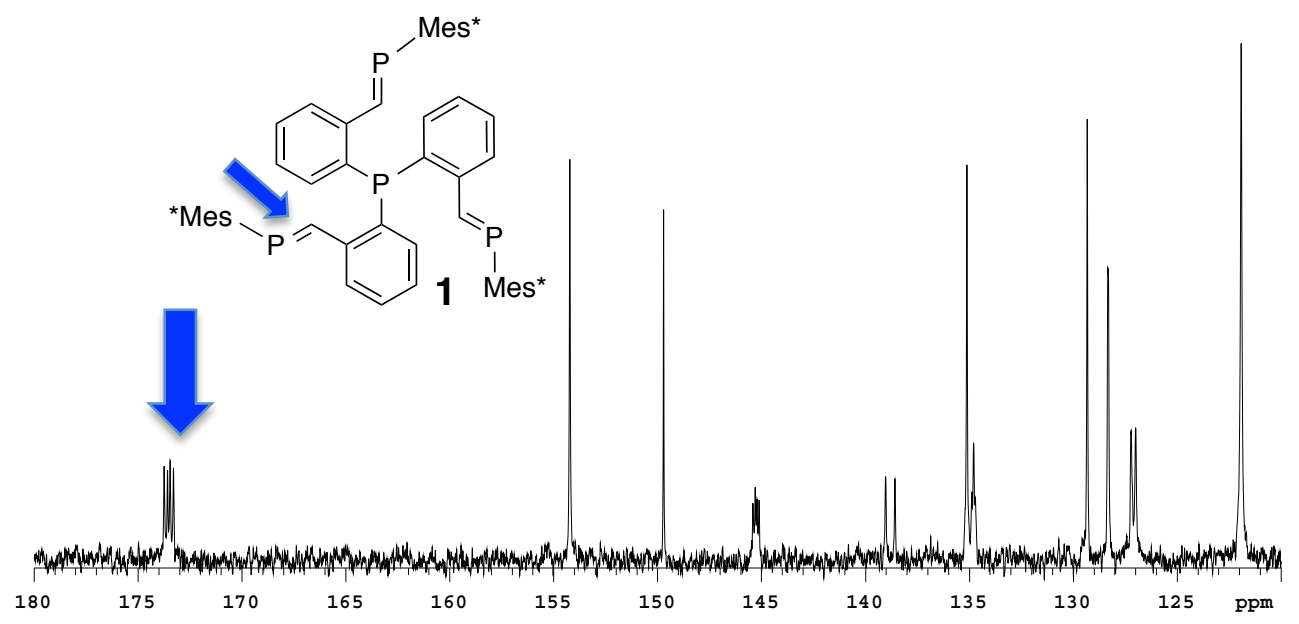




\section{Elemental Analysis}

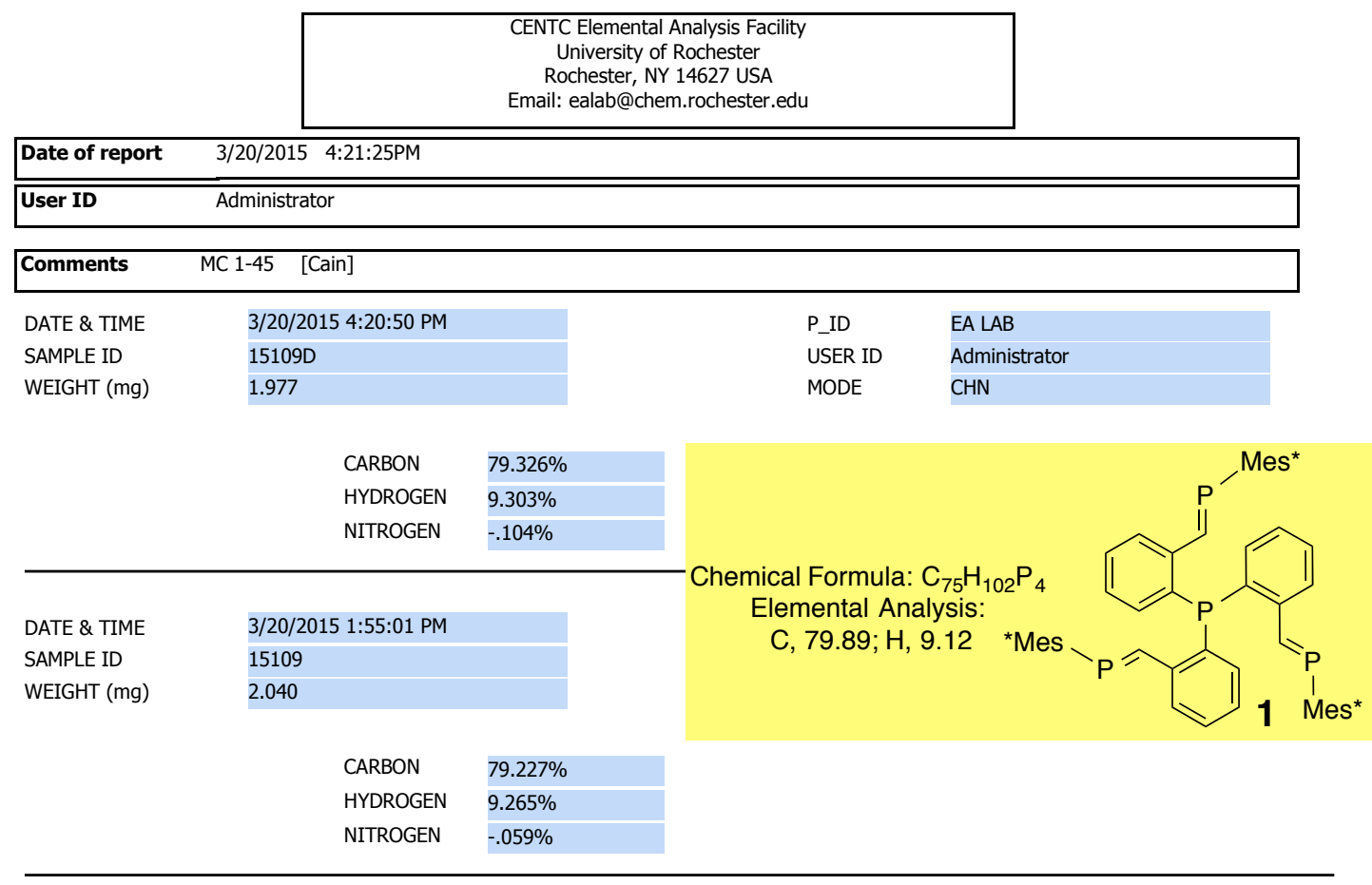

Special Handling

The sample was transferred under argon and was combusted in a tin capsule that was crimp-sealed with a die apparatus.

\section{$\underline{\text { Acknowledgment }}$}

Analytical data were obtained from the CENTC Elemental Analysis Facility at the University of Rochester, funded by NSF CHE-0650456.

Instrumentation

Microanalysis samples were weighed with a PerkinElmer Model AD-6 Autobalance and their compositions were determined with a PerkinElmer 2400 Series II Analyzer. Air-sensitive samples were handled in a VAC Atmospheres glovebox. 


\section{Metallation of 1 on $\operatorname{Rh}(I)$ and $\operatorname{Ir}(I)$ centers}
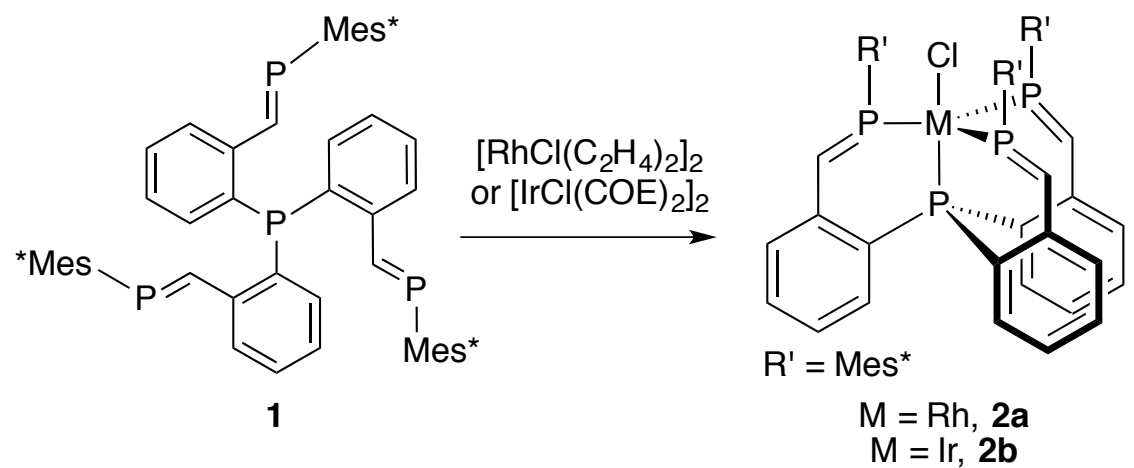

\section{Synthesis of 2a}

A solution of 1 (126 mg, $0.112 \mathrm{mmol}$ ) in $5 \mathrm{~mL}$ of benzene was added to $\left[\mathrm{RhCl}\left(\mathrm{C}_{2} \mathrm{H}_{4}\right)_{2}\right]_{2}$ (22 $\mathrm{mg}, 0.057 \mathrm{mmol}, 0.5$ equiv) producing a dark reaction mixture. The reaction mixture was stirred for $24 \mathrm{~h}$ at room temperature and then concentrated under vacuum. Subsequently, the crude product was dissolved in 3 $\mathrm{mL}$ chloroform and filtered through a Celite plug. The filtrate was layered with acetonitrile $(10 \mathrm{~mL})$ and placed in the freezer at $-35{ }^{\circ} \mathrm{C}$. After standing overnight, a dark solid precipitated (134 mg, $0.106 \mathrm{mmol}, 95 \%, 2 \mathrm{crops}$ ) from solution. X-ray quality crystals were obtained from an additional recrystallization of $10 \mathrm{mg}$ of $\mathbf{2 a}$ in $1 \mathrm{~mL}$ of chloroform layered with $3 \mathrm{~mL}$ of acetonitrile at $-35{ }^{\circ} \mathrm{C}$.

Elemental analysis data was consistently low (3 attempts) in carbon, for example: Anal. Calcd. for $\mathrm{C}_{75} \mathrm{H}_{102} \mathrm{P}_{4} \mathrm{RhCl}$ : C, 71.16; H, 8.12. Found: C, 69.87; H, 8.05. HRMS $m / z$ calcd for $\left[\mathrm{C}_{75} \mathrm{H}_{103} \mathrm{P}_{4} \mathrm{RhCl}(\mathrm{M}+\mathrm{H})^{+}\right]: 1265.5754$. Found: $1265.5740 .{ }^{31} \mathrm{P}\left\{{ }^{1} \mathrm{H}\right\} \mathrm{NMR}$ $\left(\mathrm{CDCl}_{3}\right): \delta 241.1\left(\mathrm{dd}, J_{\mathrm{Rh}-\mathrm{P}}=169, J_{\mathrm{PP}}=44 \mathrm{~Hz}, \mathrm{P}=\mathrm{C}\right), 39.2$ (overlapping doublet of quartets, $J_{\mathrm{Rh}-\mathrm{P}}=99, J_{\mathrm{PP}}=44$, apical P). ${ }^{1} \mathrm{H}$ NMR $\left(\mathrm{CDCl}_{3}\right): \delta 8.16(\mathrm{br}, 3 \mathrm{H}, \mathrm{P}=\mathrm{C}), 7.39(\mathrm{t}, J$ $=7.5 \mathrm{~Hz}, 3 \mathrm{H}, \mathrm{Ar}$ ), 7.32 (3H, Mes*), 7.27 (3H, Mes*), 7.15 (br t, $J=6 \mathrm{~Hz}, 3 \mathrm{H}, \mathrm{Ar}$ ), 7.09 $(\mathrm{t}, J=7.5 \mathrm{~Hz}, 3 \mathrm{H}, \mathrm{Ar}), 6.32(\mathrm{dd}, J=8,12 \mathrm{~Hz}, 3 \mathrm{H}, \mathrm{Ar}), 1.28(27 \mathrm{H}, t-\mathrm{Bu}), 1.25(27 \mathrm{H}, t-$ $\mathrm{Bu}), 1.08(27 \mathrm{H}, t-\mathrm{Bu}) .{ }^{13} \mathrm{C}\left\{{ }^{1} \mathrm{H}\right\} \mathrm{NMR}\left(\mathrm{CDCl}_{3}\right): \delta 155.7(\mathrm{Ar}), 151.9(\mathrm{Ar}), 150.3(\mathrm{P}=\mathrm{C})$, 149.7 (Ar), 144.4 (d, $J=12 \mathrm{~Hz}, \mathrm{Ar}$ ), 133.5 (Ar), 130.7 (br, likely two overlapping Ar signals), 129.0 (Ar), 126.4 (Ar), 122.7 (Ar), 122.3 (Ar), 115.7 (br, Ar), $39.9\left(\mathrm{CMe}_{3}\right.$ ), $38.8\left(\mathrm{CMe}_{3}\right), 34.8\left(\mathrm{CMe}_{3}\right), 34.5(t-\mathrm{Bu}), 33.3(t-\mathrm{Bu}), 31.2(t-\mathrm{Bu}) .{ }^{13} \mathrm{C}$ NMR assignments were aided by DEPT experiments. 
${ }^{31} \mathrm{P}\left\{{ }^{1} \mathrm{H}\right\} \mathrm{NMR}\left(\mathrm{CDCl}_{3}\right)$

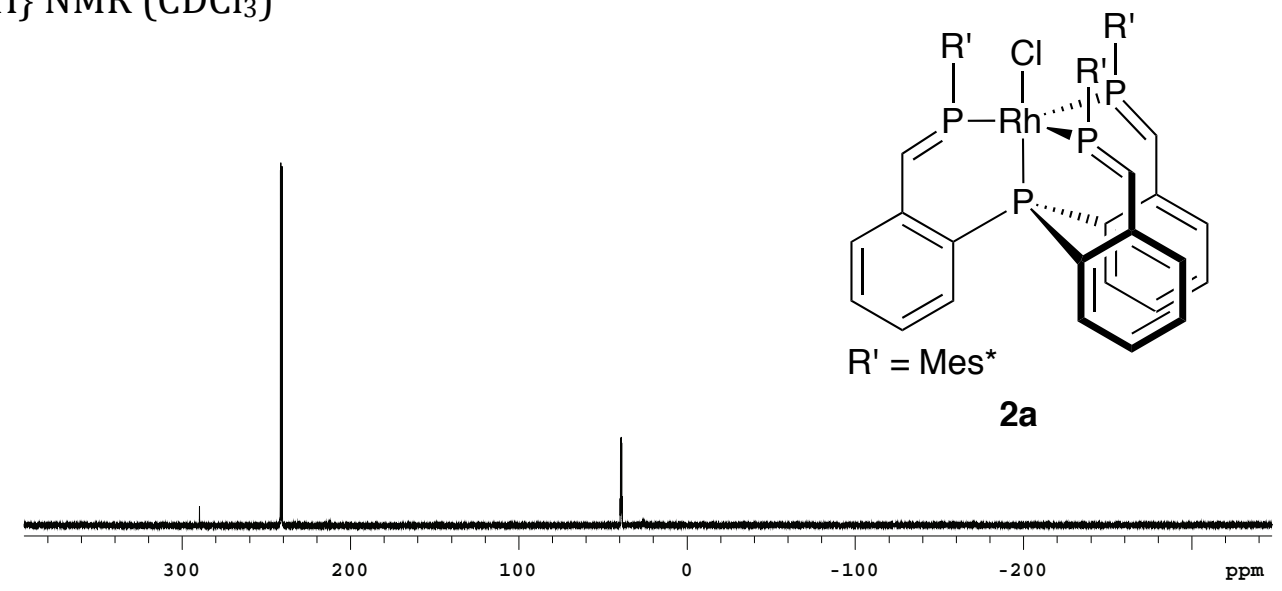

${ }^{31} \mathrm{P}\left\{{ }^{1} \mathrm{H}\right\}$ NMR $\left(\mathrm{CDCl}_{3}\right)$ : Phosphaalkene region

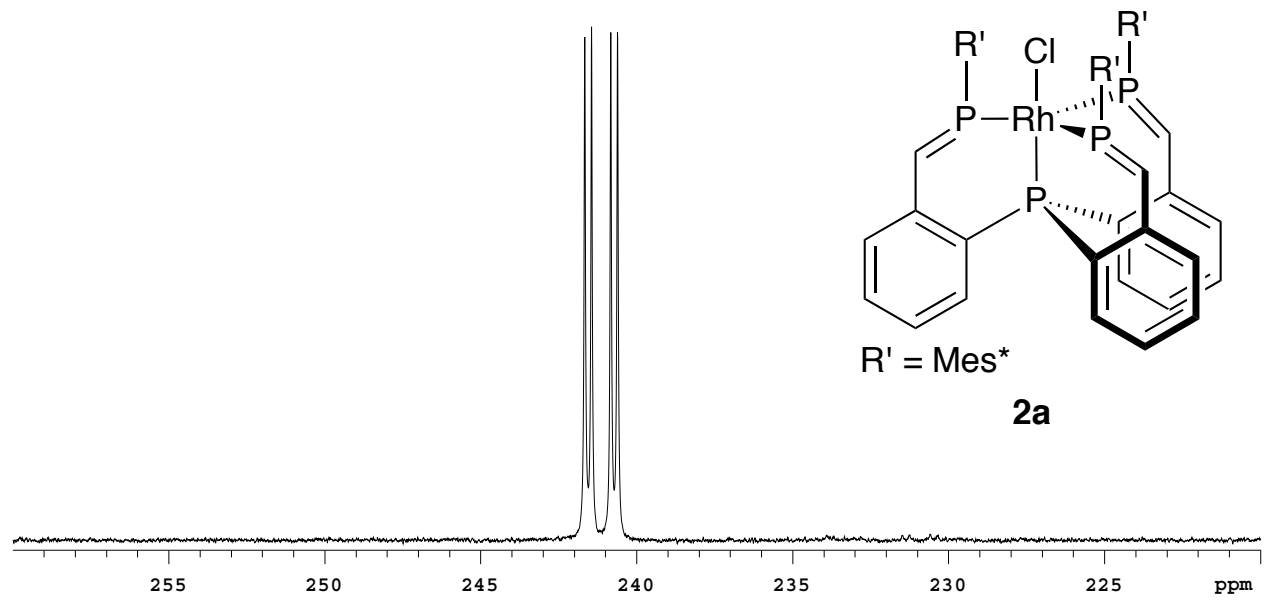


${ }^{31} \mathrm{P}\left\{{ }^{1} \mathrm{H}\right\} \mathrm{NMR}\left(\mathrm{CDCl}_{3}\right):$ Apical P region

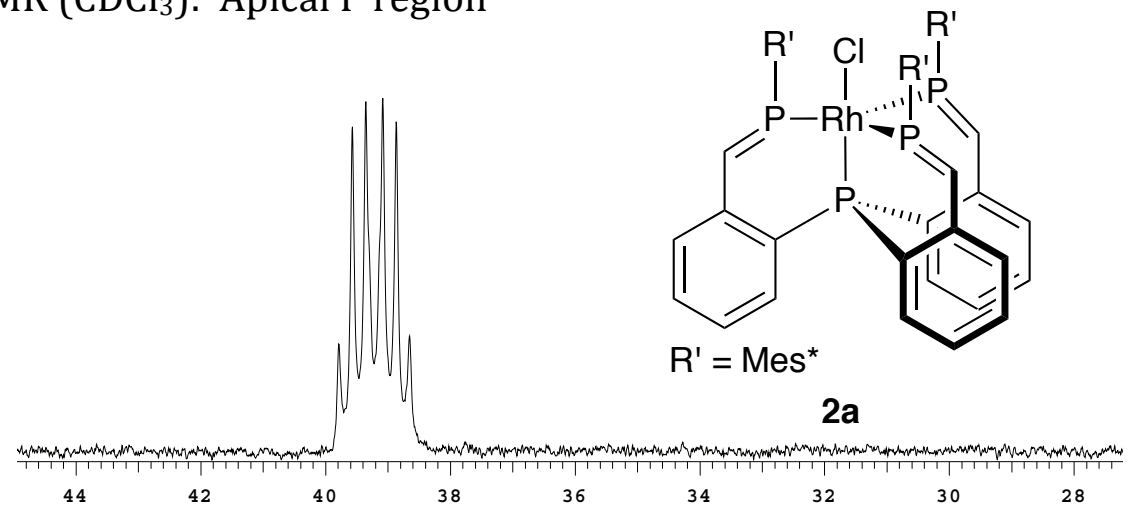

${ }^{1} \mathrm{H}$ NMR $\left(\mathrm{CDCl}_{3}\right)$ : Note three separate $t$-Bu signals suggesting the presence of restricted rotation about P-Mes* group
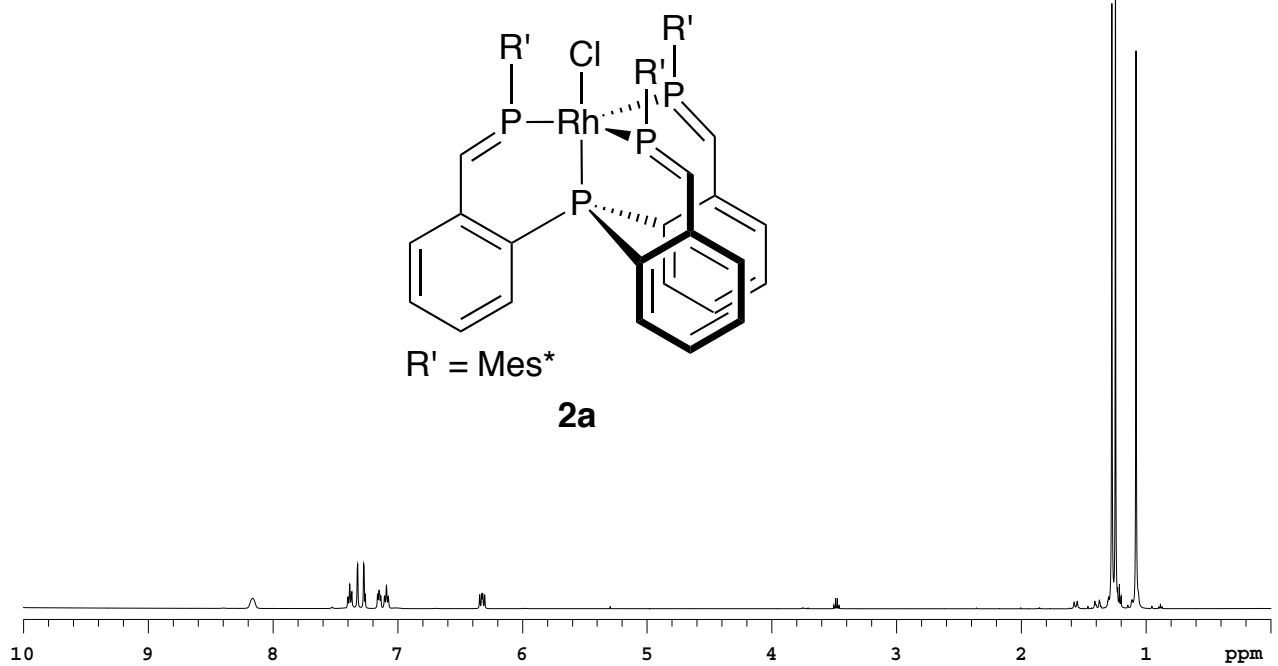
${ }^{1} \mathrm{H}$ NMR $\left(\mathrm{CDCl}_{3}\right)$ : Note two separate Mes* resonances (singlets) suggesting the presence of restricted rotation about P-Mes* group. Phosphaalkene is indicated with blue arrow.

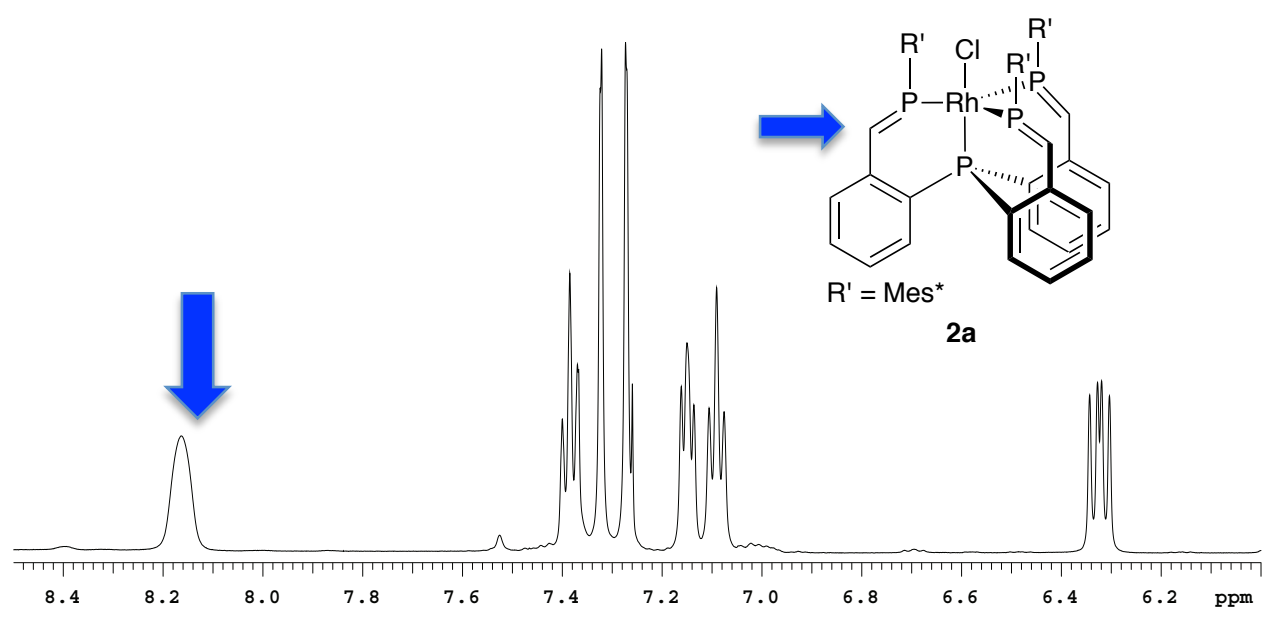

${ }^{13} \mathrm{C}\left\{{ }^{1} \mathrm{H}\right\} \mathrm{NMR}\left(\mathrm{CDCl}_{3}\right):$ Trace $\mathrm{Et}_{2} \mathrm{O}$ present

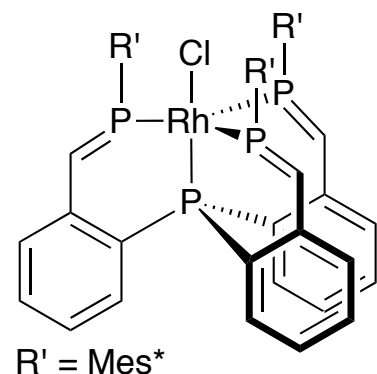

2a

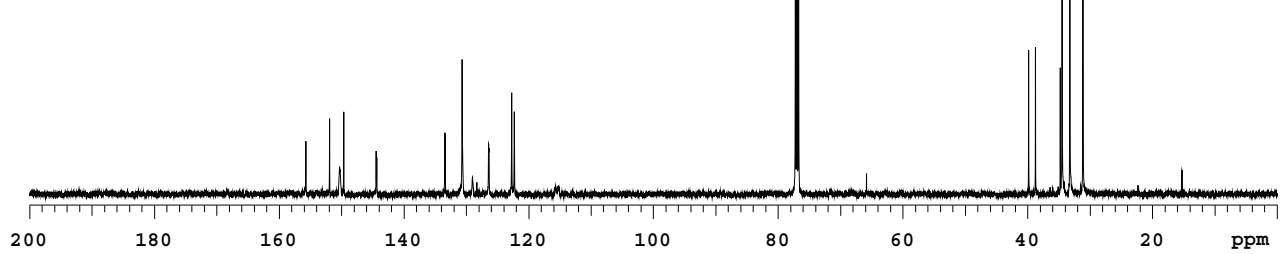


${ }^{13} \mathrm{C}\left\{{ }^{1} \mathrm{H}\right\}$ NMR $\left(\mathrm{CDCl}_{3}\right)$ : Aryl and Phosphaalkene Region

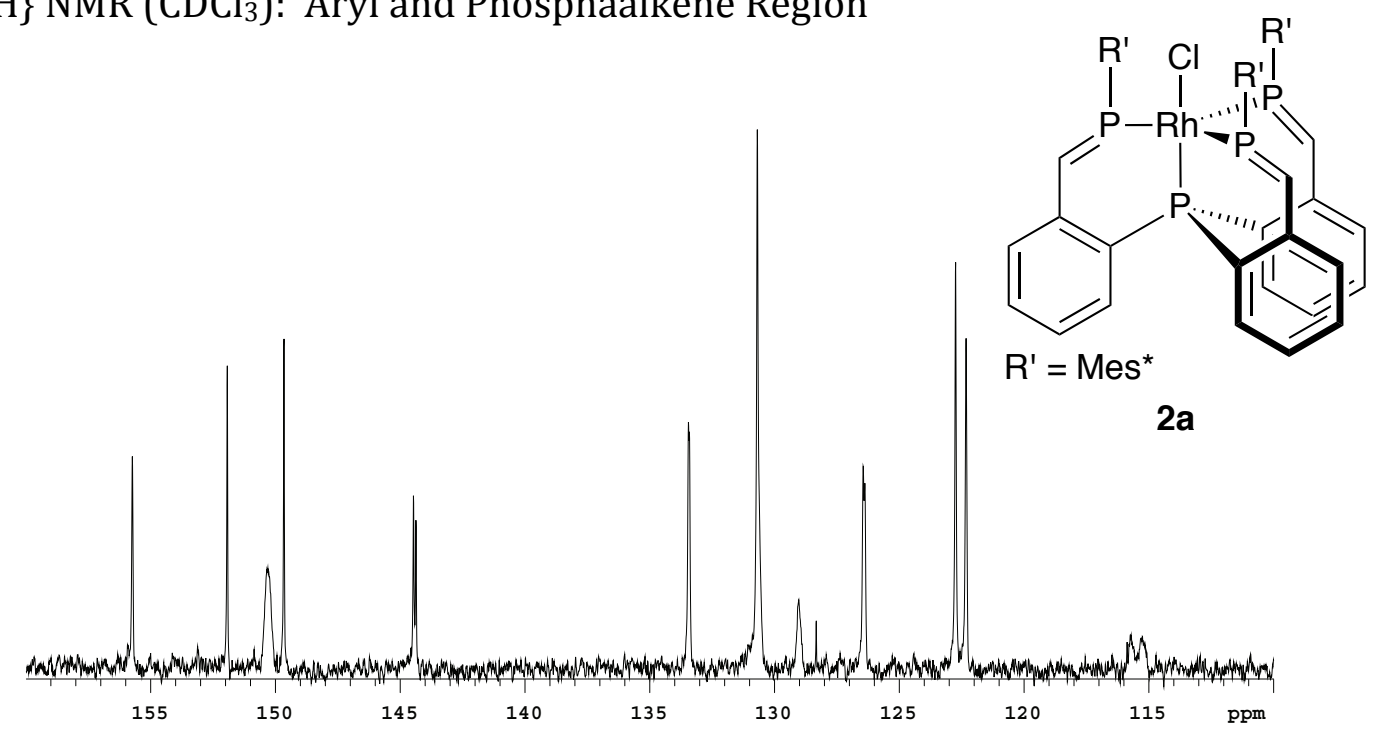

${ }^{13} \mathrm{C}\left\{{ }^{1} \mathrm{H}\right\}$ NMR $\left(\mathrm{CDCl}_{3}\right)$ : Three $t$-Bu $\left(\mathrm{CMe}_{3}\right)$ signals and three quaternary $\left(\mathrm{CMe}_{3}\right)$ signals suggesting the presence of restricted rotation about the $\mathrm{P}-\mathrm{Mes}^{*}$ bond

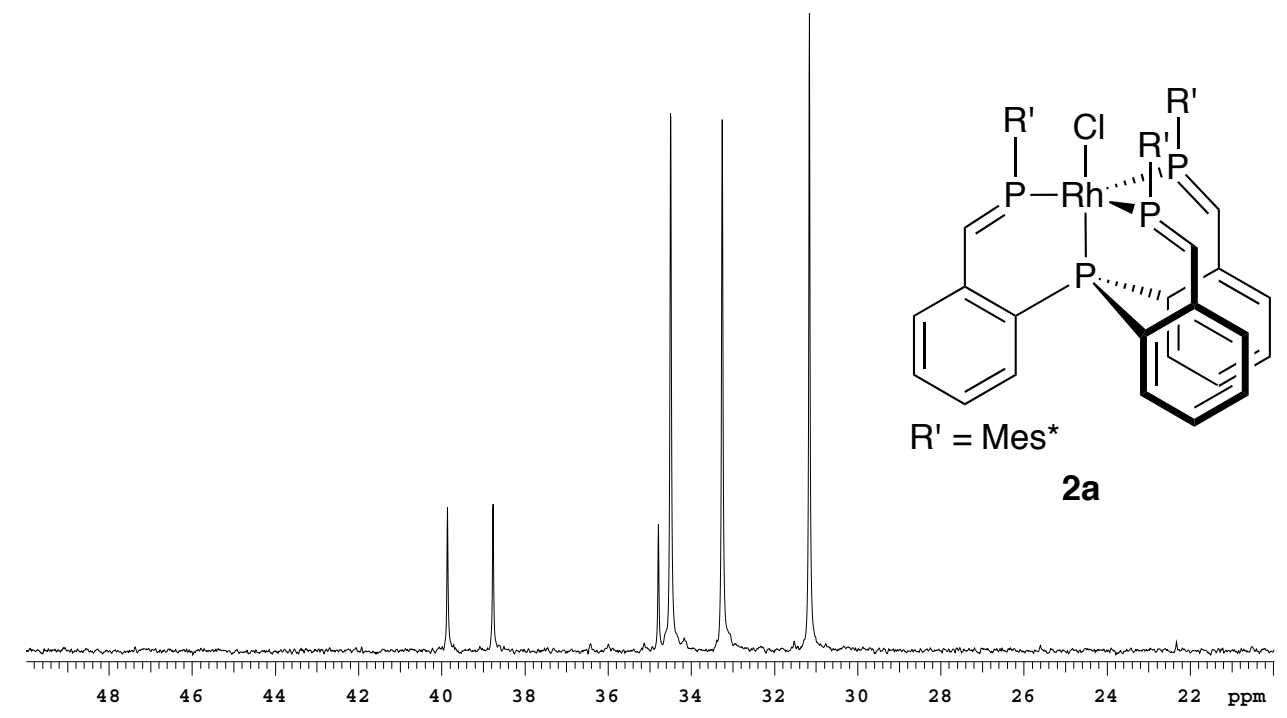




\section{Elemental analysis}

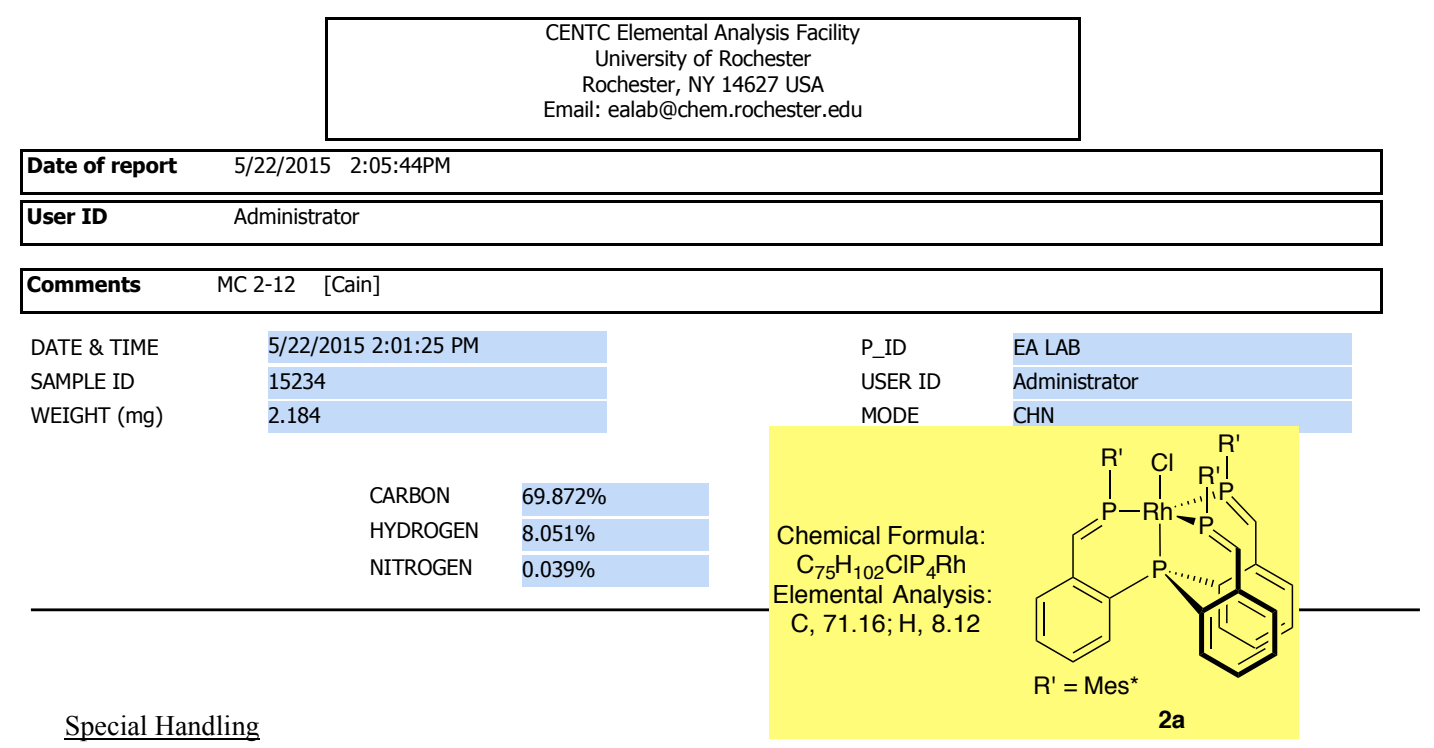

The sample was transferred under argon and was combusted in a tin capsule that was crimp-sealed with a die apparatus.

$\underline{\text { Acknowledgment }}$

Analytical data were obtained from the CENTC Elemental Analysis Facility at the University of Rochester, funded by NSF CHE-0650456.

Instrumentation

Microanalysis samples were weighed with a PerkinElmer Model AD-6 Autobalance and their compositions were determined with a PerkinElmer 2400 Series II Analyzer. Air-sensitive samples were handled in a VAC Atmospheres glovebox. 


\section{Synthesis of $2 b$}

A solution of 1 (100 mg, $0.087 \mathrm{mmol}$ ) in $4 \mathrm{~mL}$ of benzene was added to [ $\left.\mathrm{IrCl}(\mathrm{COE})_{2}\right]_{2}$ (48 $\mathrm{mg}, 0.052 \mathrm{mmol}, 0.6$ equiv) producing a dark reaction mixture. The reaction mixture was transferred to a screw-top Teflon Schlenk bomb, heated at $40{ }^{\circ} \mathrm{C}$ for 24 $\mathrm{h}$ with stirring, and then concentrated under vacuum. The crude product was dissolved in pentane and filtered through a Celite plug. The filtrate was concentrated under vacuum and the residue was washed with cold acetonitrile (-35 ${ }^{\circ} \mathrm{C}$ ) to wash away any remaining COE. The crude solid was dissolved in $1 \mathrm{~mL}$ of chloroform and layered with $1 \mathrm{~mL}$ of acetonitrile resulting in the precipitation of a dark crystalline solid at room temperature $(70 \mathrm{mg}, 0.050 \mathrm{mmol}, 58 \%, 2 \mathrm{crops})$. Dissolving $10 \mathrm{mg}$ of $\mathbf{2 b}$ in a $3 \mathrm{~mL}$ of a 1:2 solution of chloroform and acetonitrile at $35^{\circ} \mathrm{C}$ produced X-ray quality crystals.

Elemental analysis data was consistently off ( 3 attempts), for example: Anal. Calcd. for $\left[\mathrm{C}_{75} \mathrm{H}_{102} \mathrm{P} 4 \mathrm{IrCl}\right]$ : C, 66.47; H, 7.59. Found: $\mathrm{C}, 70.90 ; \mathrm{H}, 8.26$. HRMS $\mathrm{m} / \mathrm{z}$ calcd for $\left[\mathrm{C}_{75} \mathrm{H}_{103} \mathrm{P} \mathrm{P}_{4} \mathrm{IrCl}(\mathrm{M}+\mathrm{H})^{+}\right]:$1355.6328. Found: $1355.6351 .{ }^{31} \mathrm{P}\left\{{ }^{1} \mathrm{H}\right\} \mathrm{NMR}\left(\mathrm{CDCl}_{3}\right): \delta$ $211.0(\mathrm{~d}, J=34 \mathrm{~Hz}, \mathrm{P}=\mathrm{C}),-8.4$ (quartet, $J=34 \mathrm{~Hz}$, apical P). ${ }^{1} \mathrm{H}$ NMR $\left(\mathrm{CDCl}_{3}\right): \delta 8.22$ (br, 3H, P=C), $7.58(6 \mathrm{H}$, overlapping Mes*), $6.96(\mathrm{t}, J=7.5 \mathrm{~Hz}, 3 \mathrm{H}, \mathrm{Ar}), 6.85(\mathrm{t}, J=6$ $\mathrm{Hz}, 3 \mathrm{H}, \mathrm{Ar}), 6.72(\mathrm{t}, J=7.5 \mathrm{~Hz}, 3 \mathrm{H}, \mathrm{Ar}), 6.58(\mathrm{dd}, J=8,12.5 \mathrm{~Hz}, 3 \mathrm{H}, \mathrm{Ar}), 1.57(27 \mathrm{H}, t-$ $\mathrm{Bu}), 1.39(27 \mathrm{H}, t-\mathrm{Bu}), 1.31(27 \mathrm{H}, t-\mathrm{Bu}) .{ }^{13} \mathrm{C}\left\{{ }^{1} \mathrm{H}\right\} \mathrm{NMR}\left(\mathrm{CDCl}_{3}\right): \delta 155.7(\mathrm{Ar}), 151.9$ (Ar), 149.9 (Ar), 144.2 (d, J = $11 \mathrm{~Hz}, \mathrm{Ar}$ ), 140.0 (br, P=C), 133.4 (Ar), 130.5 (Ar), 130.0 (br, Ar), 127.5 (Ar), 125.4 (d, J = $10 \mathrm{~Hz}, \mathrm{Ar}$ ), 122.8 (Ar), 122.0 (Ar), 114.0 (br, Ar), $39.9\left(\mathrm{CMe}_{3}\right), 39.1\left(\mathrm{CMe}_{3}\right), 34.8\left(\mathrm{CMe}_{3}\right), 34.3(t-\mathrm{Bu}), 33.0(t-\mathrm{Bu}), 31.2(t-\mathrm{Bu}) .{ }^{13} \mathrm{C}$ NMR assignments were aided by DEPT experiments. 
${ }^{31} \mathrm{P}\left\{{ }^{1} \mathrm{H}\right\} \operatorname{NMR}\left(\mathrm{C}_{6} \mathrm{D}_{6}\right)$

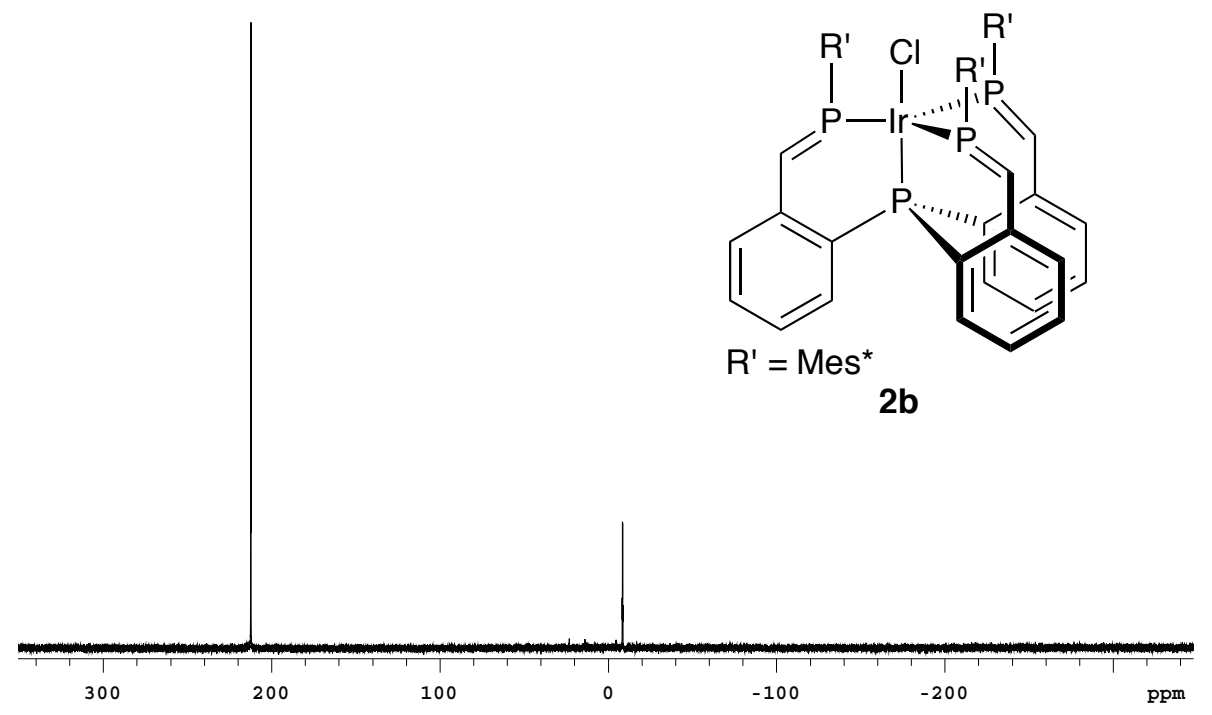

${ }^{31} \mathrm{P}\left\{{ }^{1} \mathrm{H}\right\}$ NMR $\left(\mathrm{C}_{6} \mathrm{D}_{6}\right)$ : Phosphaalkene region
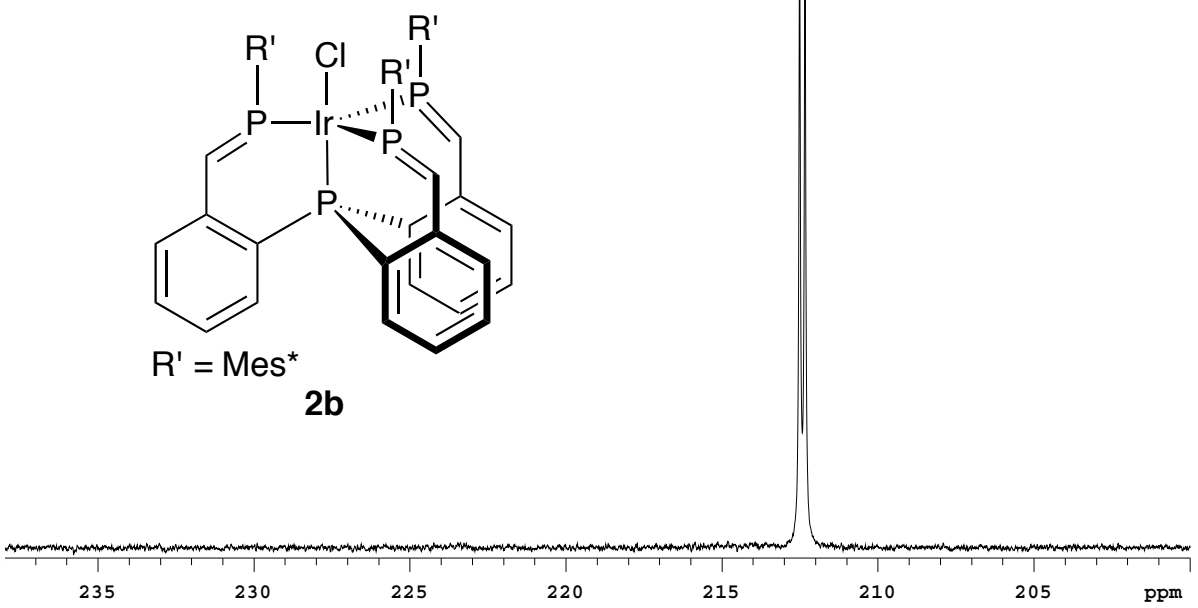
${ }^{31} \mathrm{P}\left\{{ }^{1} \mathrm{H}\right\} \operatorname{NMR}\left(\mathrm{C}_{6} \mathrm{D}_{6}\right):$ Apical P region

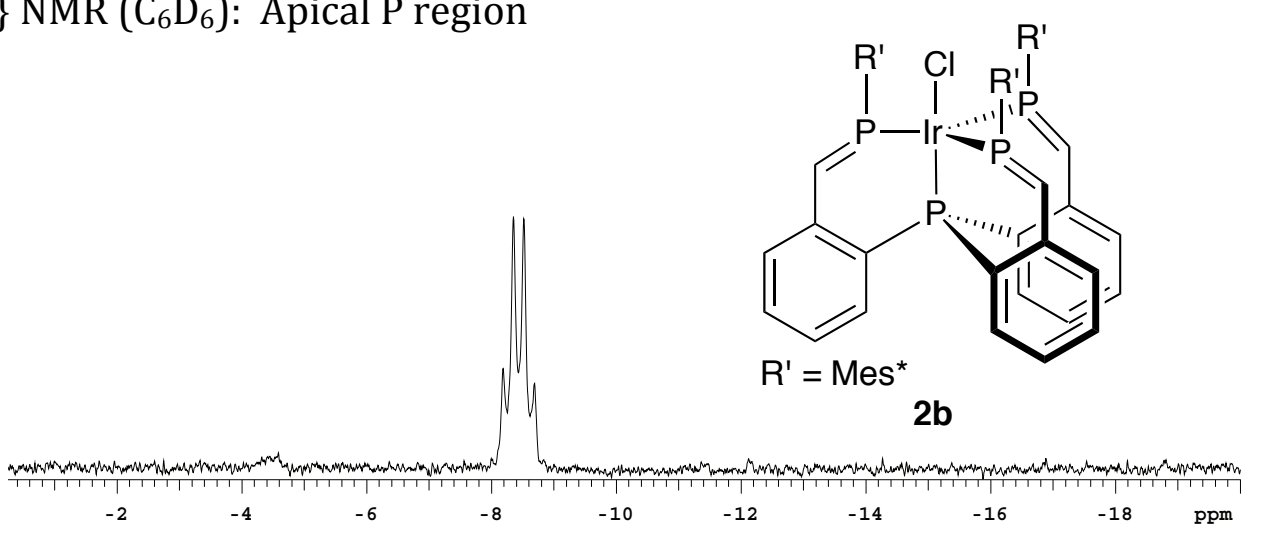

${ }^{1} \mathrm{H} \mathrm{NMR}\left(\mathrm{C}_{6} \mathrm{D}_{6}\right)$ : Note three separate $t$ - $\mathrm{Bu}$ signals suggesting the presence of restricted rotation about $\mathrm{P}-\mathrm{Mes}^{*}$ group

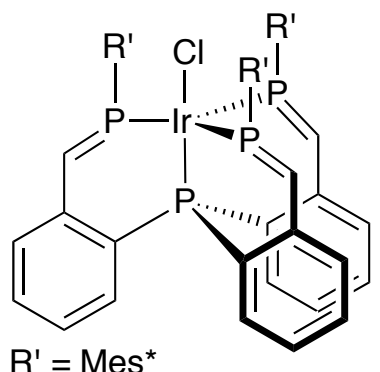

$2 b$

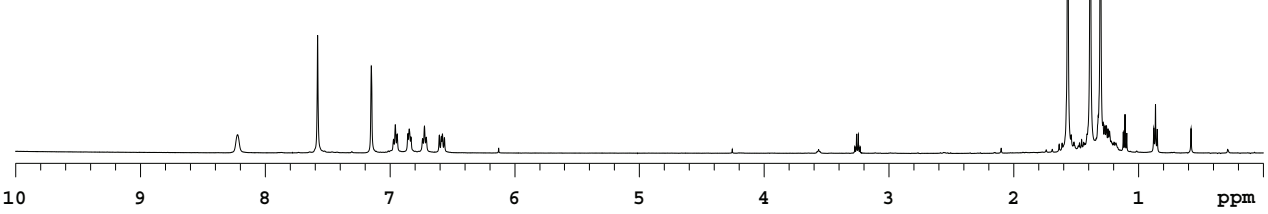


${ }^{1} \mathrm{H}$ NMR $\left(\mathrm{C}_{6} \mathrm{D}_{6}\right)$ : Aryl and phosphaalkene region

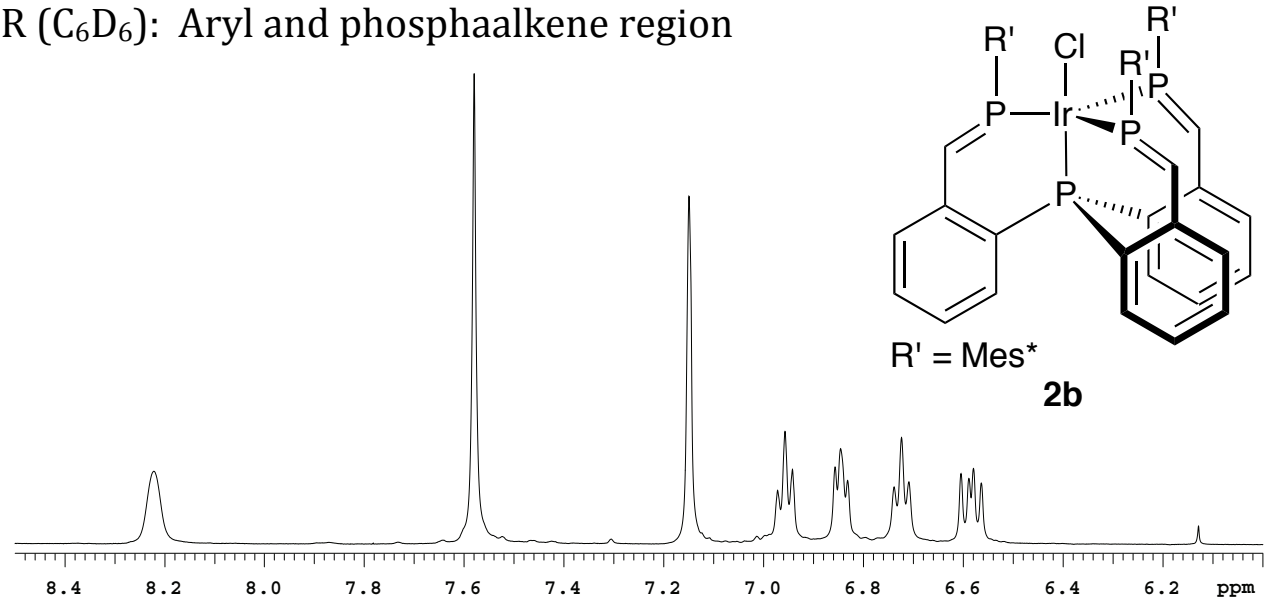

${ }^{13} \mathrm{C}\left\{{ }^{1} \mathrm{H}\right\}$ NMR $\left(\mathrm{CDCl}_{3}\right): \mathrm{Et}_{2} \mathrm{O}$ present

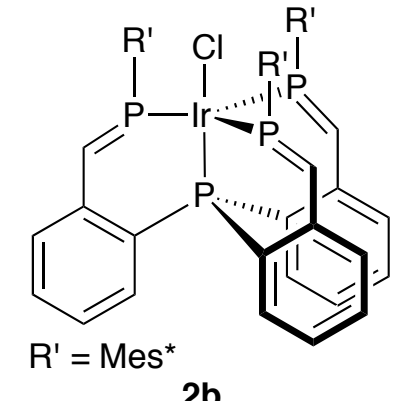

$2 b$

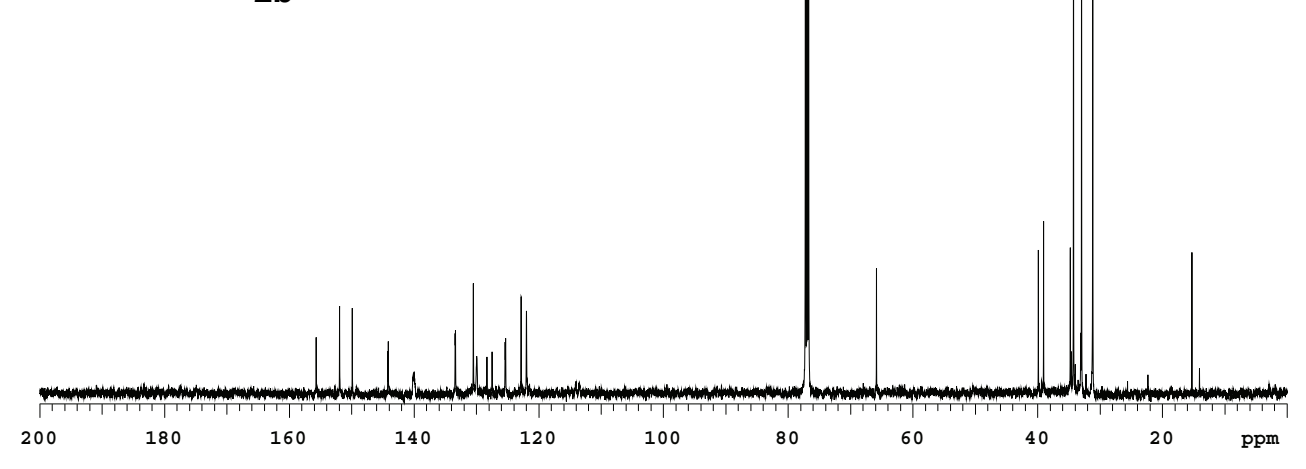


${ }^{13} \mathrm{C}\left\{{ }^{1} \mathrm{H}\right\}$ NMR $\left(\mathrm{CDCl}_{3}\right)$ : Aryl and Phosphaalkene region

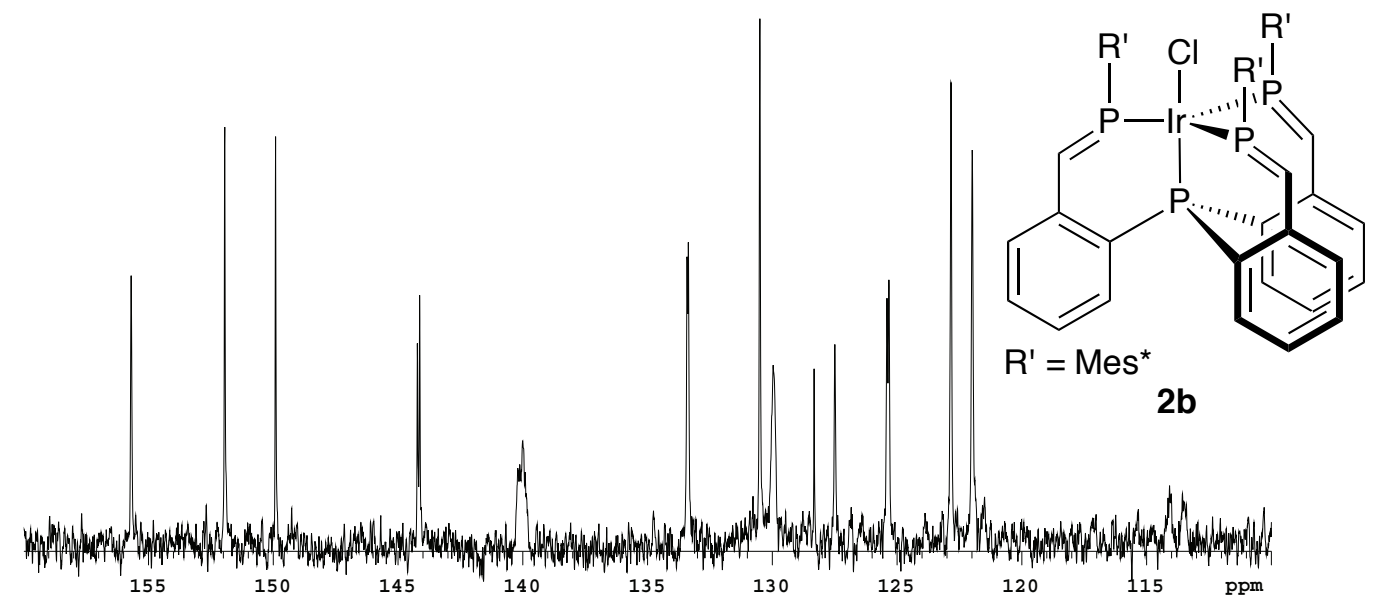

${ }^{13} \mathrm{C}\left\{{ }^{1} \mathrm{H}\right\} \mathrm{NMR}\left(\mathrm{CDCl}_{3}\right)$ : Three $t$-Bu $\left(\mathrm{CMe}_{3}\right)$ signals and three quaternary $\left(\mathrm{CMe}_{3}\right)$ signals suggesting the presence of restricted rotation about the P-Mes* bond

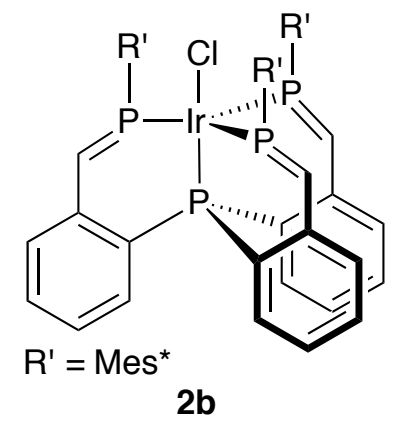

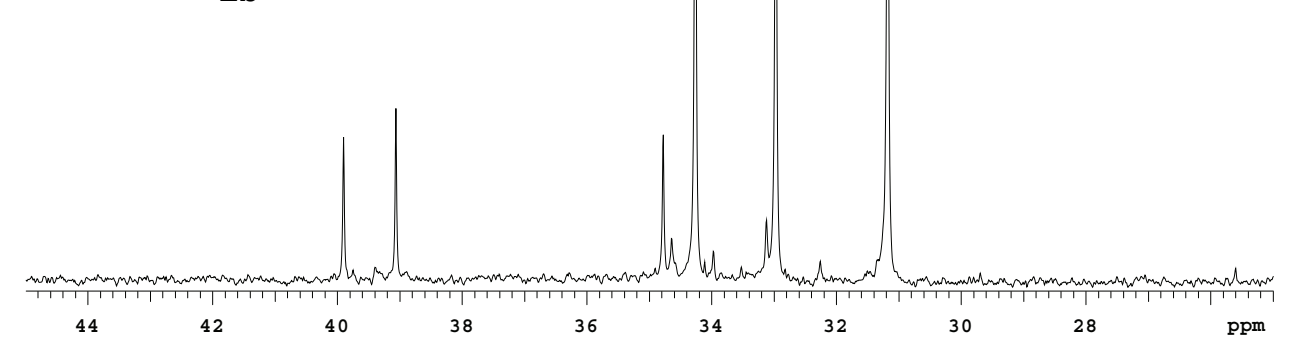




\section{Elemental Analysis}

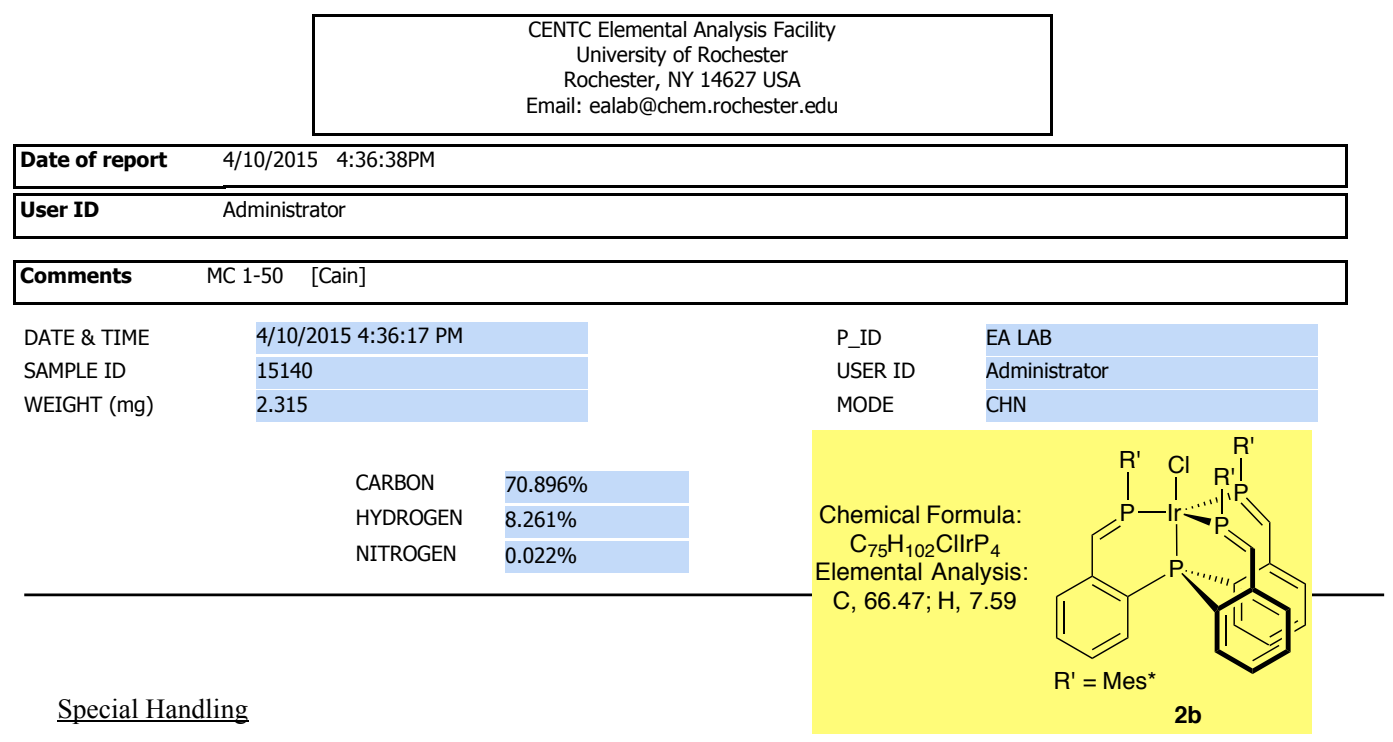

The sample was transferred under argon and was combusted in a tin capsule that was crimp-sealed with a die apparatus.

\section{Acknowledgment}

Analytical data were obtained from the CENTC Elemental Analysis Facility at the University of Rochester, funded by NSF CHE-0650456.

$\underline{\text { Instrumentation }}$

Microanalysis samples were weighed with a PerkinElmer Model AD-6 Autobalance and their compositions were determined with a PerkinElmer 2400 Series II Analyzer. Air-sensitive samples were handled in a VAC

Atmospheres glovebox. 


\section{Synthesis of 3}

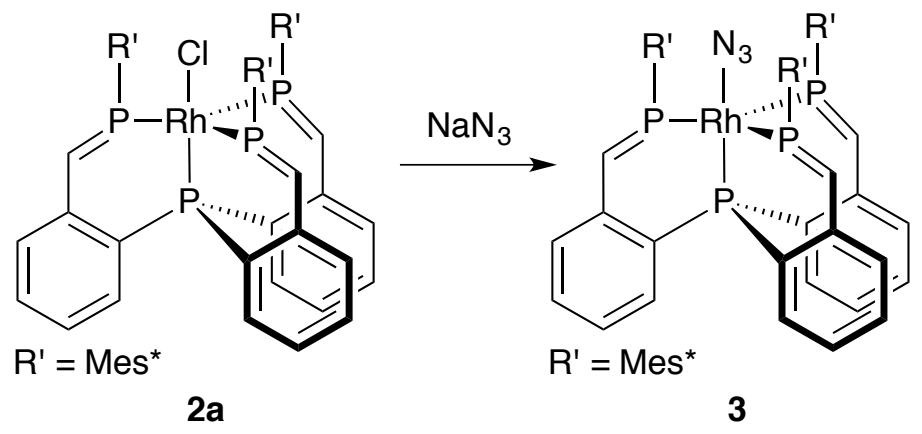

A solution of $2 \mathbf{a}\left(70 \mathrm{mg}, 0.055 \mathrm{mmol}\right.$ ) in $3 \mathrm{~mL}$ of chloroform was added to $\mathrm{NaN}_{3}$ (54 $\mathrm{mg}, 0.83 \mathrm{mmol}, 15$ equiv) and stirred for $72 \mathrm{~h}$ at room temperature. The progress of the reaction can be tracked by ${ }^{1} \mathrm{H}$ NMR spectroscopy $\left(\mathrm{CDCl}_{3},[\mathrm{Rh}]-\mathrm{Cl}\right.$ phosphaalkene signal: $\delta 8.19$ to $[\mathrm{Rh}]-\mathrm{N}_{3}$ phosphaalkene signal: $\left.\delta 7.89\right)$. After full conversion to the azide complex, the dark reaction mixture was filtered through a Celite plug, and the filtrate was concentrated under vacuum affording the product as a dark brown solid (69 mg, 0.54 mmol, 98\%). Anal. Calcd. for $\mathrm{C}_{75} \mathrm{H}_{102} \mathrm{P}_{4} \mathrm{RhN}_{3}$ : C, 70.79; $\mathrm{H}, 8.08 ; \mathrm{N}, 3.30$. Found: C, 70.52; H, 8.37; N, 3.15. HRMS $m / z$ calcd for $\left[\mathrm{C}_{75} \mathrm{H}_{102} \mathrm{P}_{4} \mathrm{RhN}_{3}\right]$ : 1271.6079. Found: 1271.6071. ${ }^{31} \mathrm{P}\left\{{ }^{1} \mathrm{H}\right\} \mathrm{NMR}\left(\mathrm{CDCl}_{3}\right): \delta 230.3\left(\mathrm{dd}, J_{\mathrm{Rh}-\mathrm{P}}=168, J_{\mathrm{PP}}=48 \mathrm{~Hz}, \mathrm{P}=\mathrm{C}\right)$, 37.0 (overlapping doublet of quartets, $J_{\mathrm{Rh}-\mathrm{P}}=97, J_{\mathrm{PP}}=48 \mathrm{~Hz}$, apical P). ${ }^{1} \mathrm{H}$ NMR $\left(\mathrm{CDCl}_{3}\right): \delta 7.86(\mathrm{br}, 3 \mathrm{H}, \mathrm{P}=\mathrm{C}), 7.39-7.34$ (overlapping, 9H, 2 Mes* and Ar), $7.09(\mathrm{t}, J=$ $7.5 \mathrm{~Hz}, 3 \mathrm{H}, \mathrm{Ar}$ ), 7.06 (t, J=7.5 Hz, 3H, Ar), 6.25 (dd, J = 7.5, $12 \mathrm{~Hz}, 3 \mathrm{H}, \mathrm{Ar}$ ), 1.45 (27H, $t$-Bu), $1.29(27 \mathrm{H}, t-\mathrm{Bu}), 1.06(27 \mathrm{H}, t-\mathrm{Bu}) .{ }^{13} \mathrm{C}\left\{{ }^{1} \mathrm{H}\right\} \mathrm{NMR}\left(\mathrm{CDCl}_{3}\right): \delta 155.4(\mathrm{Ar}), 153.3$ (Ar), 152.9 (br, P=C), 150.1 (Ar), 144.3 (d, J = 12 Hz, Ar), 133.1 (Ar), 132.6 (br, Ar), 130.9 (two overlapping Ar signals), 126.8 (d, $J=8 \mathrm{~Hz}, \mathrm{Ar}$ ), 123.2 (Ar), 122.9 (Ar), 116.1 (br), $39.5\left(\mathrm{CMe}_{3}\right), 38.6\left(\mathrm{CMe}_{3}\right), 34.8\left(C \mathrm{Me}_{3}\right), 34.0(t-\mathrm{Bu}), 33.9(t-\mathrm{Bu}), 31.2(t-$ $\mathrm{Bu}$ ). ${ }^{13} \mathrm{C}$ NMR assignments were aided by DEPT experiments. The ${ }^{15} \mathrm{~N}$-labeled isotopologue ${ }^{15} \mathrm{~N}-3$ was synthesized in an analogous fashion. ${ }^{15} \mathrm{~N}\left\{{ }^{1} \mathrm{H}\right\} \mathrm{NMR}\left(\mathrm{CDCl}_{3}\right.$, $\mathrm{RT}): \delta-242.2 .{ }^{15} \mathrm{~N}\left\{{ }^{1} \mathrm{H}\right\} \mathrm{NMR}\left(\mathrm{CDCl}_{3},-60{ }^{\circ} \mathrm{C}\right): \delta-246.5$ (singlet, $\left.\mathrm{N}_{x}\right),-346.2\left(\mathrm{dd}, J_{\mathrm{Rh}-\mathrm{N}}=\right.$ $32, J_{\mathrm{P}-\mathrm{N}}=15 \mathrm{~Hz}, \mathrm{~N}_{a}$ ). 
${ }^{31} \mathrm{P}\left\{{ }^{1} \mathrm{H}\right\} \operatorname{NMR}\left(\mathrm{CDCl}_{3}\right)$

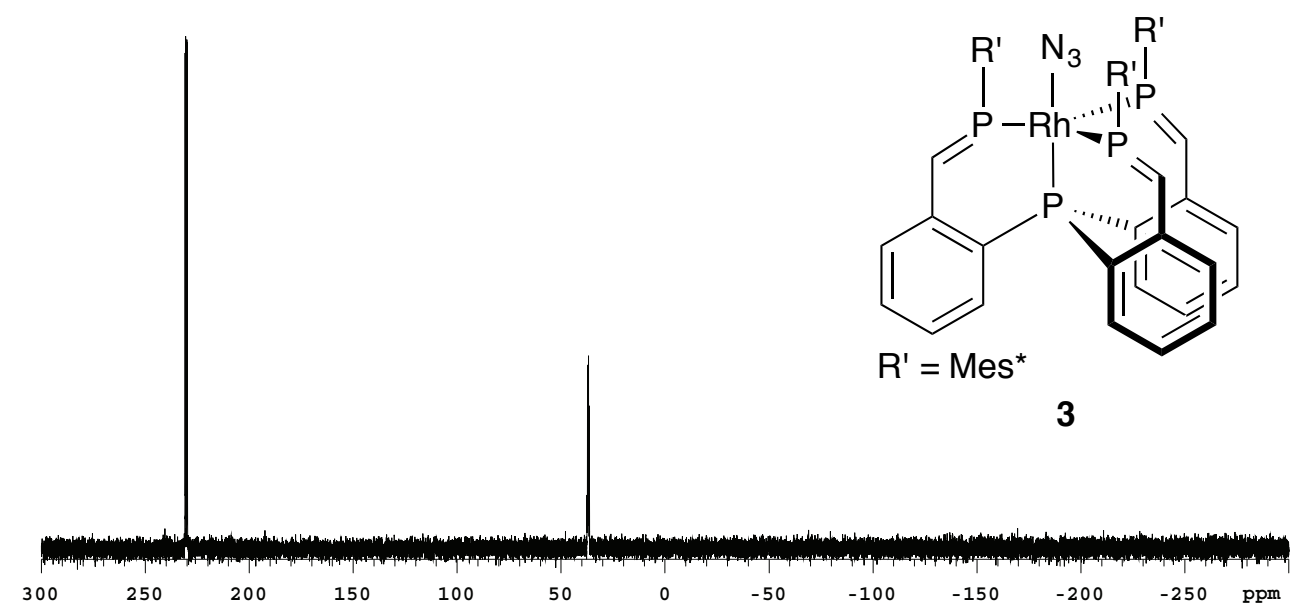

${ }^{31} \mathrm{P}\left\{{ }^{1} \mathrm{H}\right\} \mathrm{NMR}\left(\mathrm{CDCl}_{3}\right)$ : Phosphaalkene region

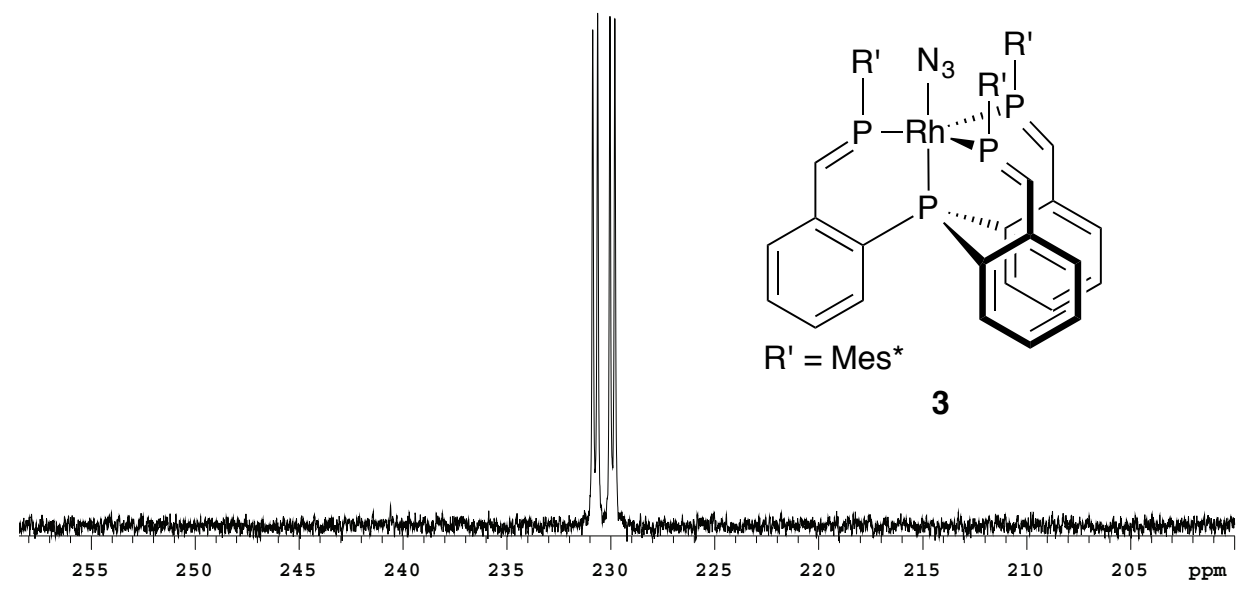

${ }^{31} \mathrm{P}\left\{{ }^{1} \mathrm{H}\right\} \mathrm{NMR}\left(\mathrm{CDCl}_{3}\right)$ : Apical P region

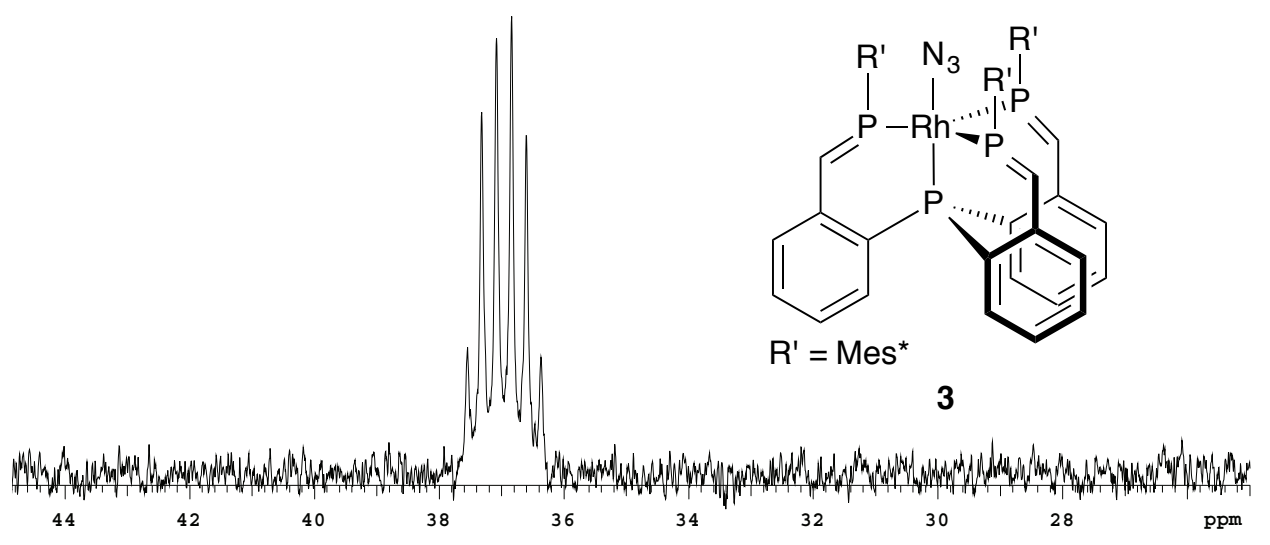


${ }^{1} \mathrm{H} \mathrm{NMR}\left(\mathrm{CDCl}_{3}\right)$ : Note three separate $t$-Bu signals suggesting the presence of restricted rotation about $\mathrm{P}-\mathrm{Mes}^{*}$ group

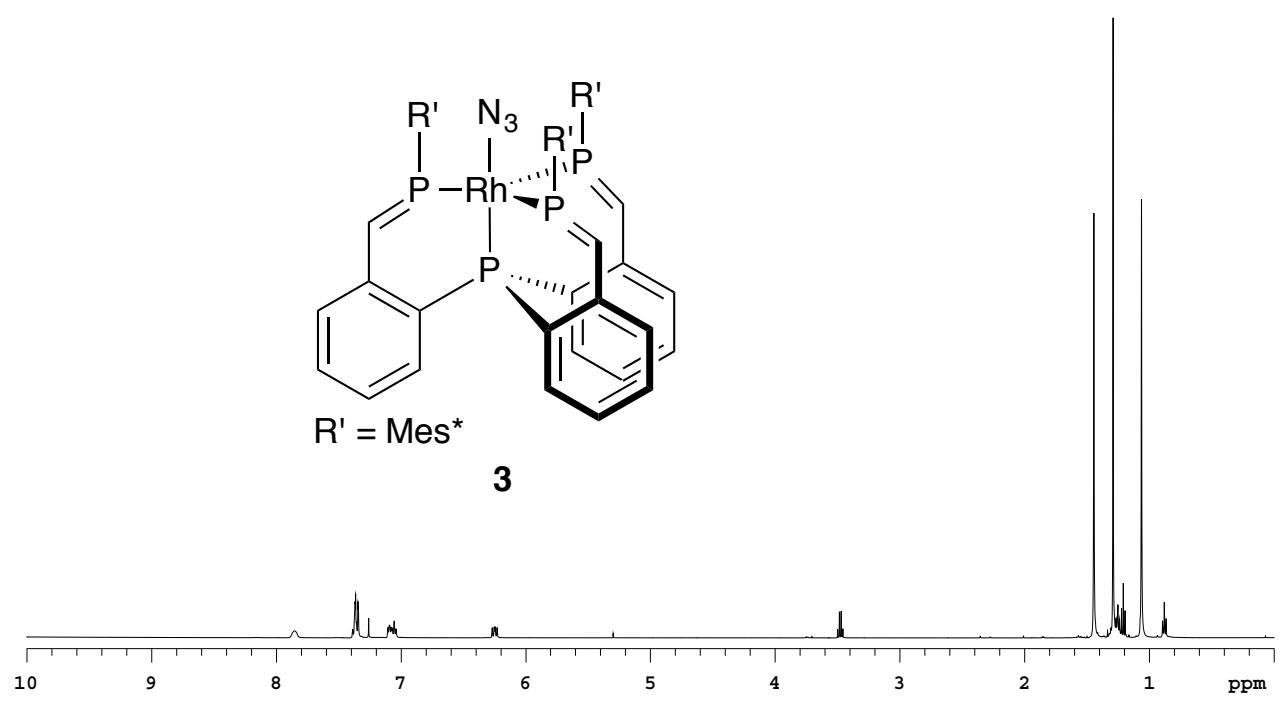

${ }^{1} \mathrm{H}$ NMR $\left(\mathrm{CDCl}_{3}\right)$ : Aryl and Phosphaalkene region

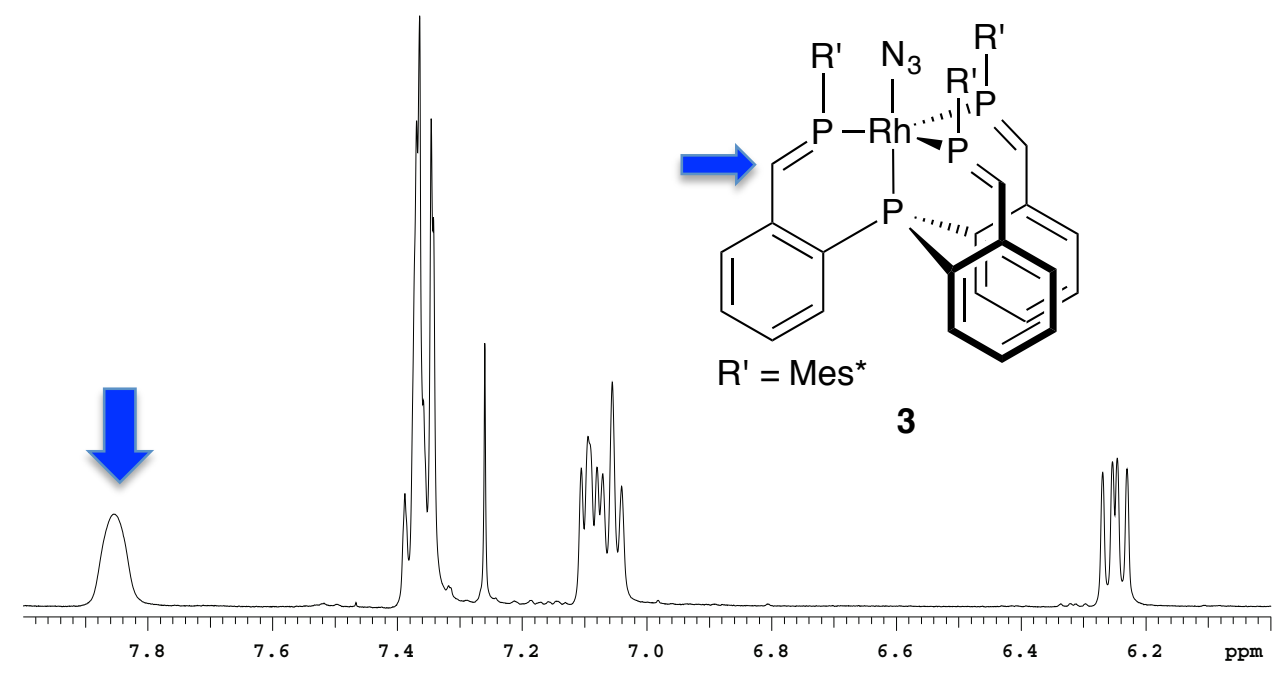


${ }^{13} \mathrm{C}\left\{{ }^{1} \mathrm{H}\right\}$ NMR $\left(\mathrm{CDCl}_{3}\right)$ : Note three separate $t$-Bu signals suggesting the presence of restricted rotation about P-Mes* group

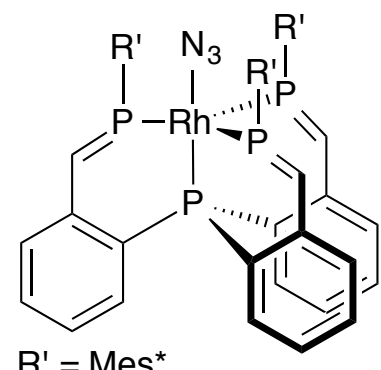

3

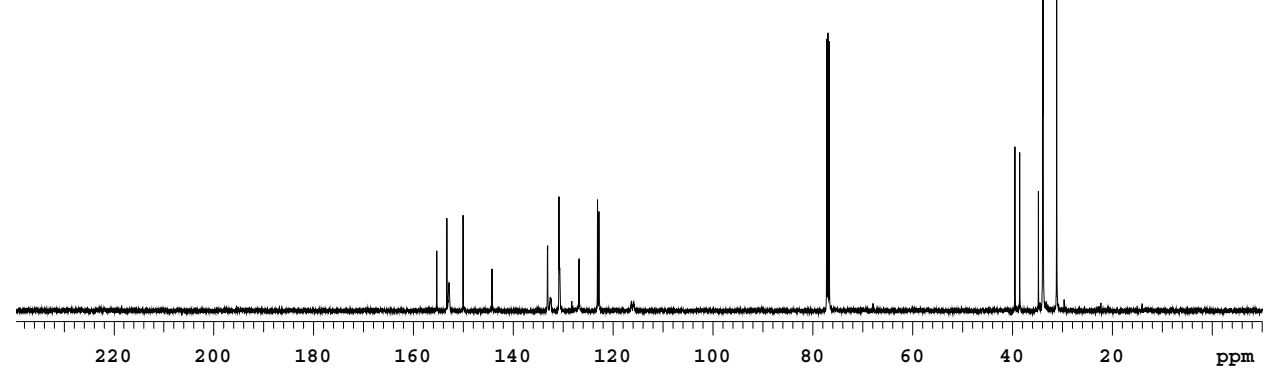




\section{IR spectra of 3 and ${ }^{15} \mathrm{~N}-3$}

(Note: Our IR spectrometer does not know what day/time it is, see upper left of the spectrum)

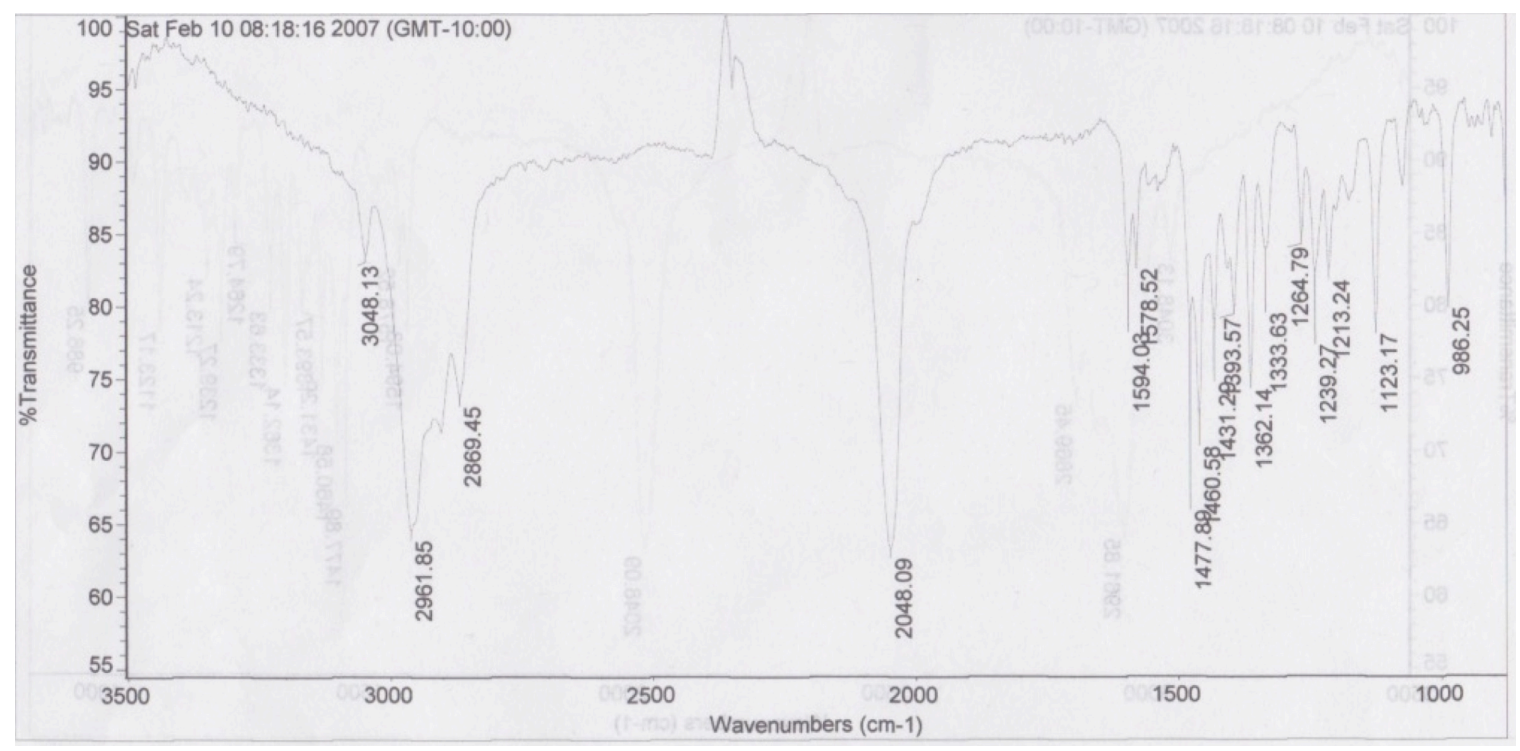

$\mathrm{Rh}-\mathrm{N}_{3}=2048 \mathrm{~cm}^{-1}$

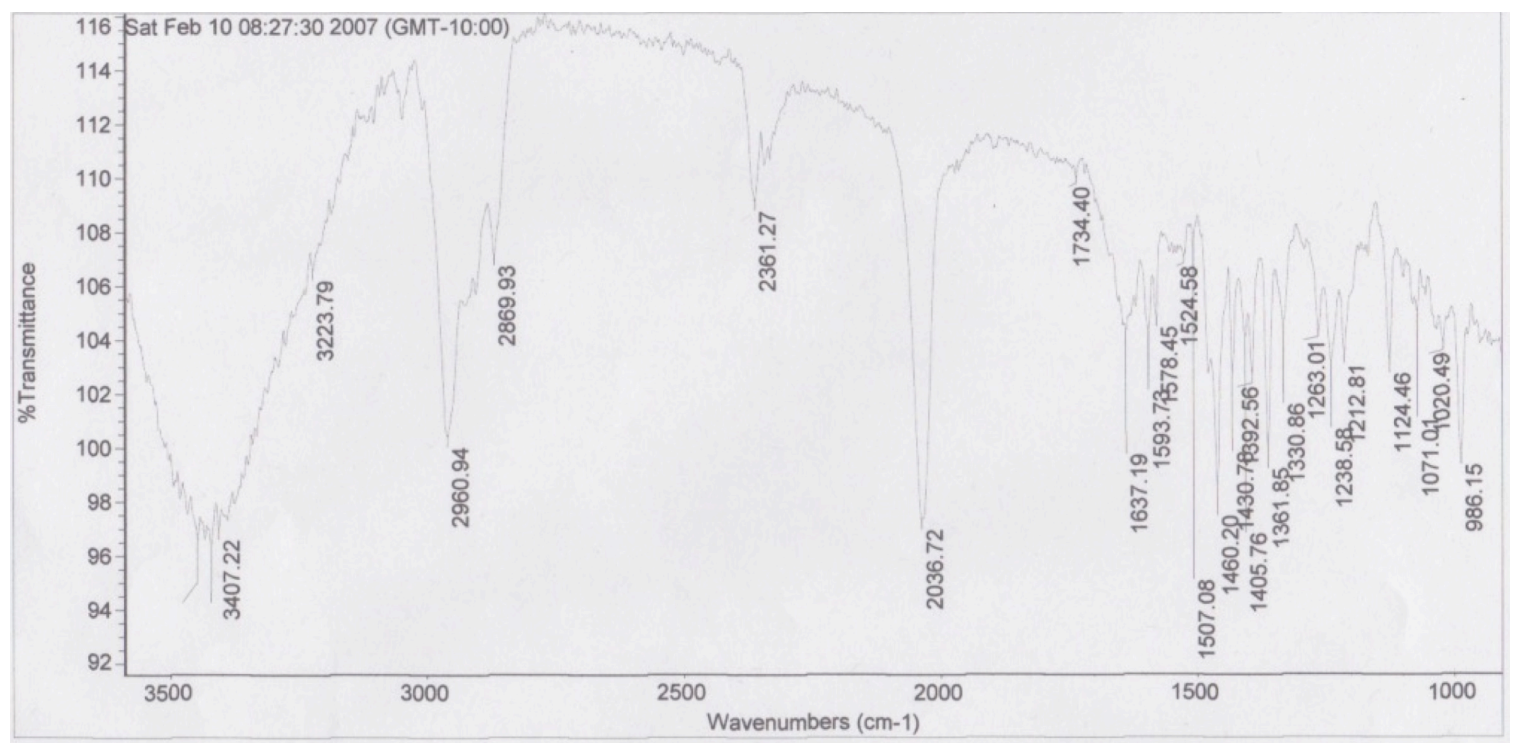

$R \mathbf{R}-{ }^{15} \mathrm{~N}_{3}=2037 \mathrm{~cm}^{-1}$ 
${ }^{15} \mathrm{~N}\left\{{ }^{1} \mathrm{H}\right\} \mathrm{NMR}\left(\mathrm{CDCl}_{3}, \mathrm{RT}\right)$

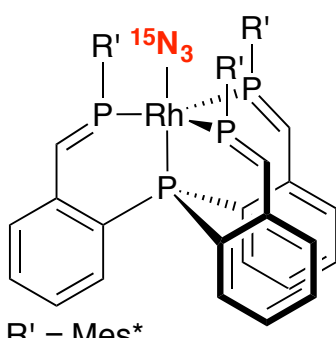

${ }^{15} \mathrm{~N}-3$
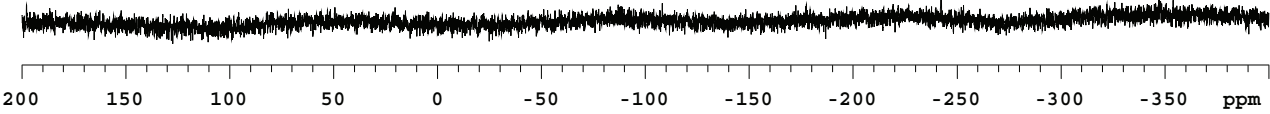

${ }^{15} \mathrm{~N}\left\{{ }^{1} \mathrm{H}\right\} \mathrm{NMR}\left(\mathrm{CDCl}_{3}, \mathrm{RT}\right):$ Zoomed in

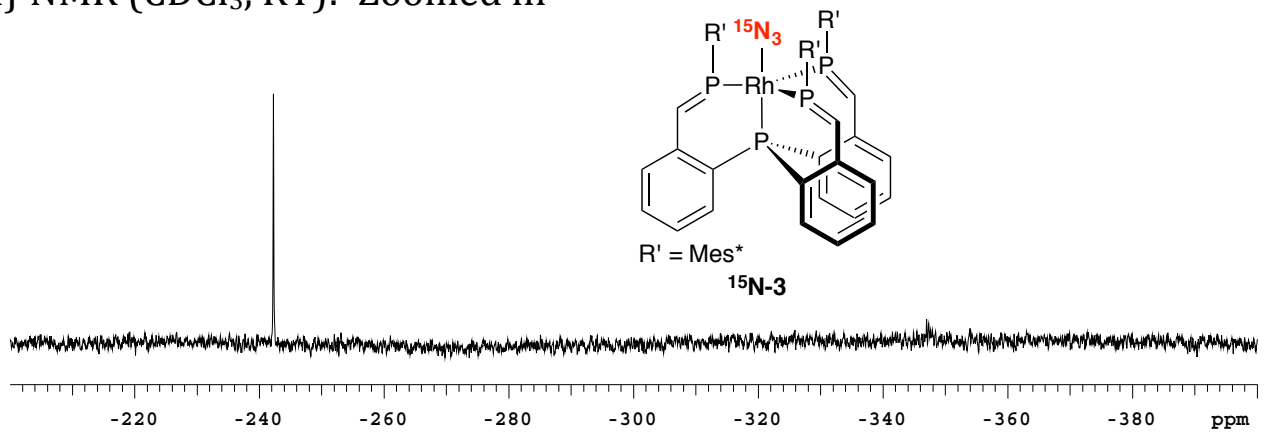

${ }^{15} \mathrm{~N}\left\{{ }^{1} \mathrm{H}\right\} \mathrm{NMR}\left(\mathrm{CDCl}_{3},-60^{\circ} \mathrm{C}\right)$

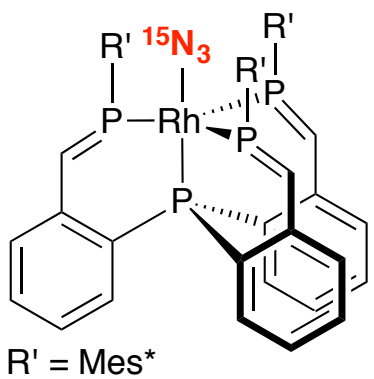

$15 \mathrm{~N}-3$

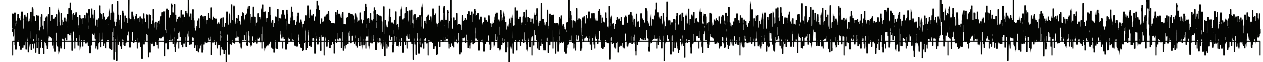


${ }^{15} \mathrm{~N}\left\{{ }^{1} \mathrm{H}\right\}$ NMR $\left(\mathrm{CDCl}_{3},-60^{\circ} \mathrm{C}\right):$ Zoomed in

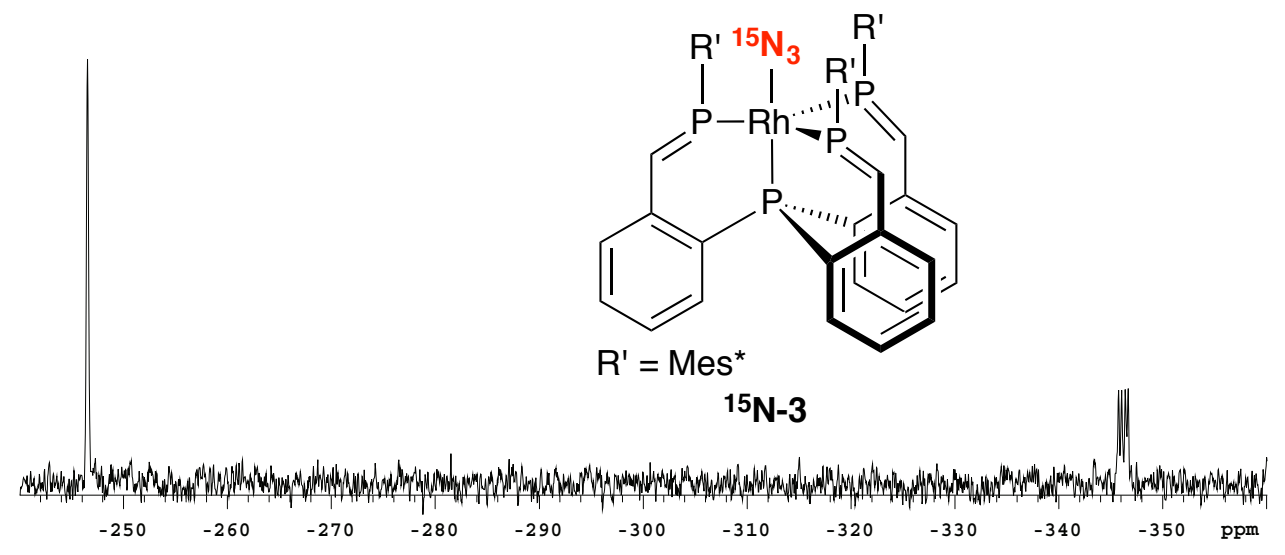

${ }^{31} \mathrm{P}\left\{{ }^{1} \mathrm{H}\right\} \mathrm{NMR}\left(\mathrm{CDCl}_{3}, \mathrm{RT}\right):$ Phosphaalkene $\mathrm{P}$ with ${ }^{15} \mathrm{~N}$ labeled azide

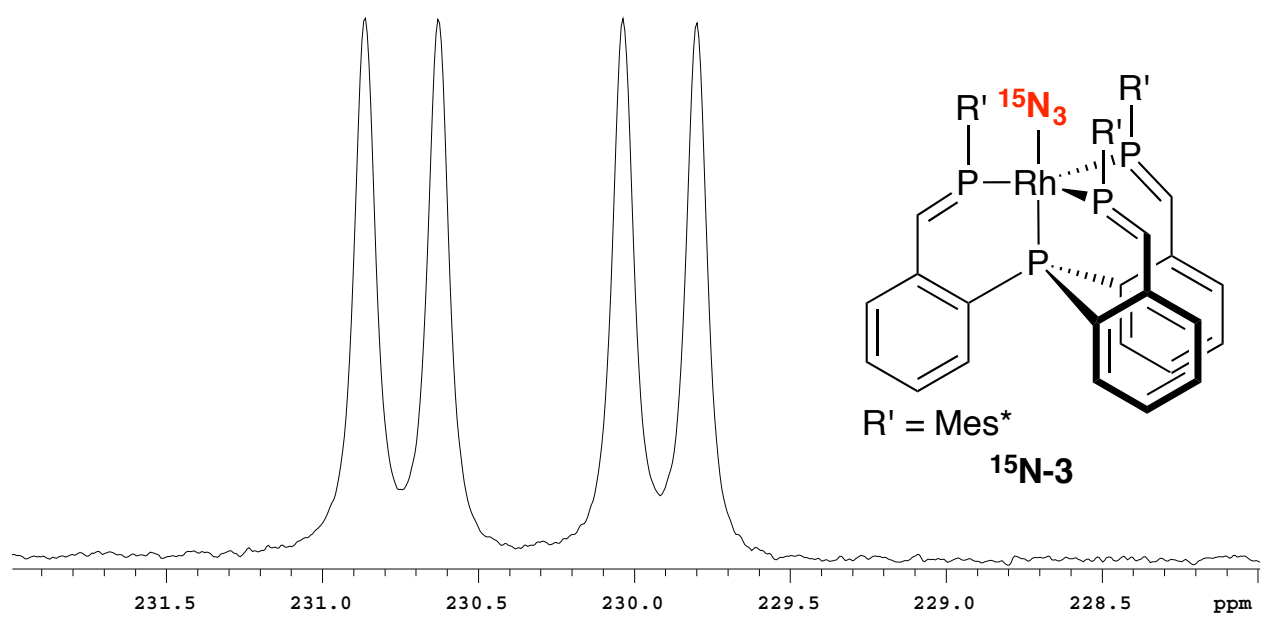


${ }^{31} \mathrm{P}\left\{{ }^{1} \mathrm{H}\right\}$ NMR $\left(\mathrm{CDCl}_{3}, \mathrm{RT}\right):$ Apical $\mathrm{P}$ with ${ }^{15} \mathrm{~N}$ labeled azide

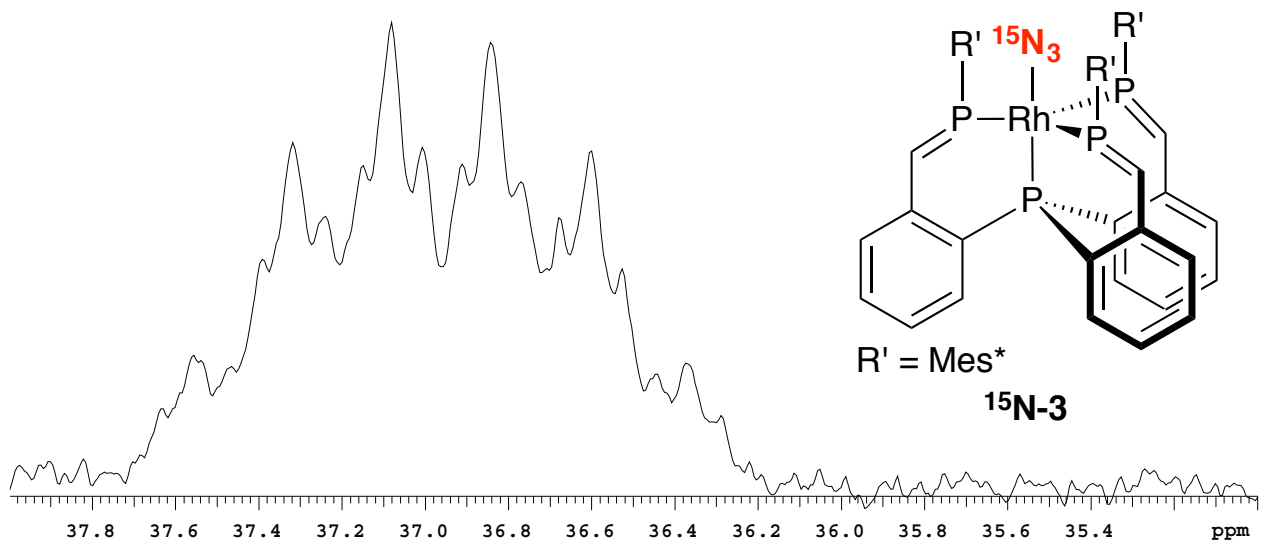




\section{Elemental Analysis}

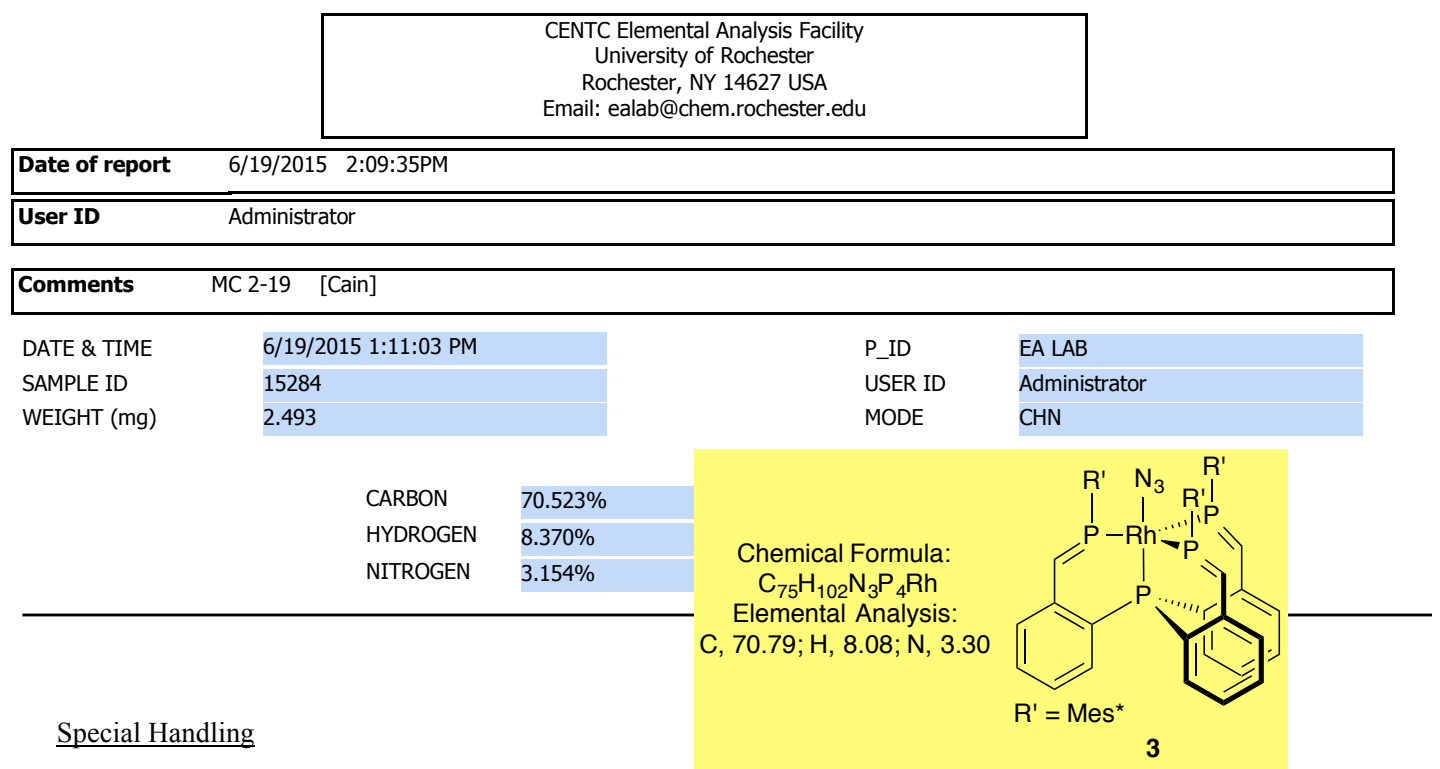

The sample was transferred under argon and was combusted in a tin capsule that was crimp-sealed with a die apparatus.

\section{$\underline{\text { Acknowledgment }}$}

Analytical data were obtained from the CENTC Elemental Analysis Facility at the University of Rochester, funded by NSF CHE-0650456.

Instrumentation

Microanalysis samples were weighed with a PerkinElmer Model AD-6 Autobalance and their compositions were determined with a PerkinElmer 2400 Series II Analyzer. Air-sensitive samples were handled in a VAC Atmospheres glovebox. 


\section{Synthesis of 4}

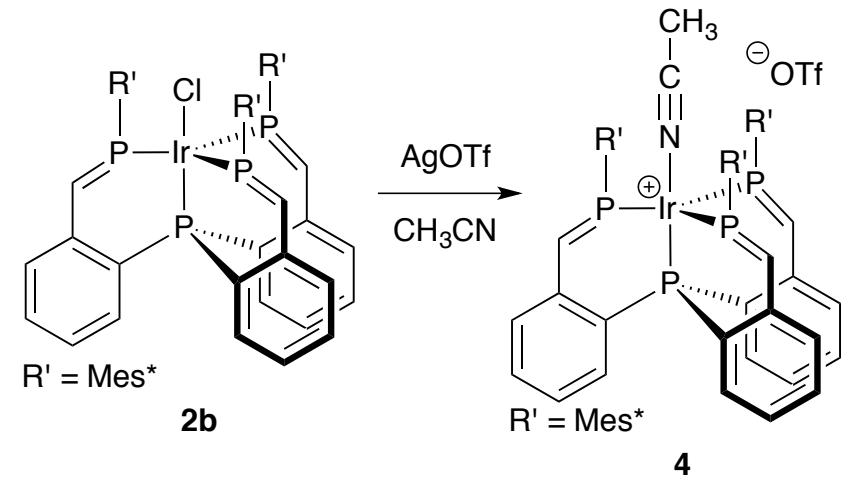

A solution of $\mathbf{2 b}(36 \mathrm{mg}, 0.027 \mathrm{mmol})$ in $1 \mathrm{~mL}$ of benzene with three drops of acetonitrile was added to solid AgOTf ( $8 \mathrm{mg}, 0.032 \mathrm{mmol}, 1.2$ equiv) and stirred for $1 \mathrm{~h}$ at room temperature. The resulting dark red solution was filtered through a Celite plug. The filtrate was layered with pentane and placed in the freezer at $-35{ }^{\circ} \mathrm{C}$ resulting in the precipitation of a dark red solid $(22 \mathrm{mg}, 0.015 \mathrm{mmol}, 55 \%)$. HRMS $m / z$ calcd for $\left[\mathrm{C}_{77} \mathrm{H}_{105} \mathrm{IrNP}_{4}(\mathrm{M}-\mathrm{NCMe})^{+}\right]: 1360.6827$. Found: 1360.6849 . ${ }^{31} \mathrm{P}\left\{{ }^{1} \mathrm{H}\right\}$ NMR ( $\left.\mathrm{C}_{6} \mathrm{D}_{6}\right): \delta 198.2(\mathrm{~d}, J=37 \mathrm{~Hz}, \mathrm{P}=\mathrm{C}$ ), -15.0 (quartet, $J=37 \mathrm{~Hz}$, apical P). An unidentified impurity is present at $24.9 \mathrm{ppm}$ (singlet). ${ }^{1} \mathrm{H}$ NMR $\left(\mathrm{C}_{6} \mathrm{D}_{6}\right): \delta 8.23(\mathrm{br}$, $3 \mathrm{H}, \mathrm{P}=\mathrm{C}), 7.75\left(3 \mathrm{H}, \mathrm{Mes}^{*}\right), 7.57\left(3 \mathrm{H}, \mathrm{Mes}^{*}\right), 6.95(\mathrm{t}, J=7.5 \mathrm{~Hz}, 3 \mathrm{H}, \mathrm{Ar}), 6.69$ (overlapping, 6H, $2 \mathrm{Ar}$ ), 6.32 (dd, $J=7.5,13 \mathrm{~Hz}, 3 \mathrm{H}, \mathrm{Ar}$ ), 2.39 (3H, NCMe), $1.47(27 \mathrm{H}$, $t$-Bu), $1.37(27 \mathrm{H}, t-\mathrm{Bu}), 1.20(27 \mathrm{H}, t-\mathrm{Bu}) .{ }^{13} \mathrm{C}\left\{{ }^{1} \mathrm{H}\right\} \mathrm{NMR}\left(\mathrm{C}_{6} \mathrm{D}_{6}\right): \delta 154.7(\mathrm{Ar}), 153.5$ (Ar), 153.0 (Ar), 144.6 (br, P=C), 143.6 (d, J = $11 \mathrm{~Hz}, \mathrm{Ar}$ ), 133.5 (d, J = $7 \mathrm{~Hz}, \mathrm{Ar}$ ), 131.8 (Ar), 130.9 (br, Ar), 124.5 ( $\mathrm{Ar}), 124.0(\mathrm{Ar}), 39.9\left(\mathrm{CMe}_{3}\right), 39.3\left(\mathrm{CMe}_{3}\right), 35.5\left(\mathrm{CMe}_{3}\right)$, $34.1(t-\mathrm{Bu}), 32.9(t-\mathrm{Bu}), 31.5(t-\mathrm{Bu}), 4.4(\mathrm{NCMe}) .{ }^{19} \mathrm{~F}\left\{{ }^{1} \mathrm{H}\right\}$ NMR $\left(\mathrm{C}_{6} \mathrm{D}_{6}\right):-77.8$ (OTf). Note: Some of the Ar peaks are obscured by the strong $\mathrm{C}_{6} \mathrm{D}_{6} / \mathrm{C}_{6} \mathrm{H}_{6}$ signal. The ${ }^{13} \mathrm{C}\left\{{ }^{1} \mathrm{H}\right\}$ and ${ }^{1} \mathrm{H}$ NMR are complicated by the fact that complex 4 is somewhat unstable; prolonged exposure to vacuum, the presence of $\mathrm{CHCl}_{3}$, and even standing in solution resulted in unidentified decomposition pathways. 
${ }^{31} \mathrm{P}\left\{{ }^{1} \mathrm{H}\right\}$ NMR $\left(\mathrm{C}_{6} \mathrm{D}_{6}\right)$ : The unidentified impurity is labeled with a red asterisk $\left({ }^{*}\right)$

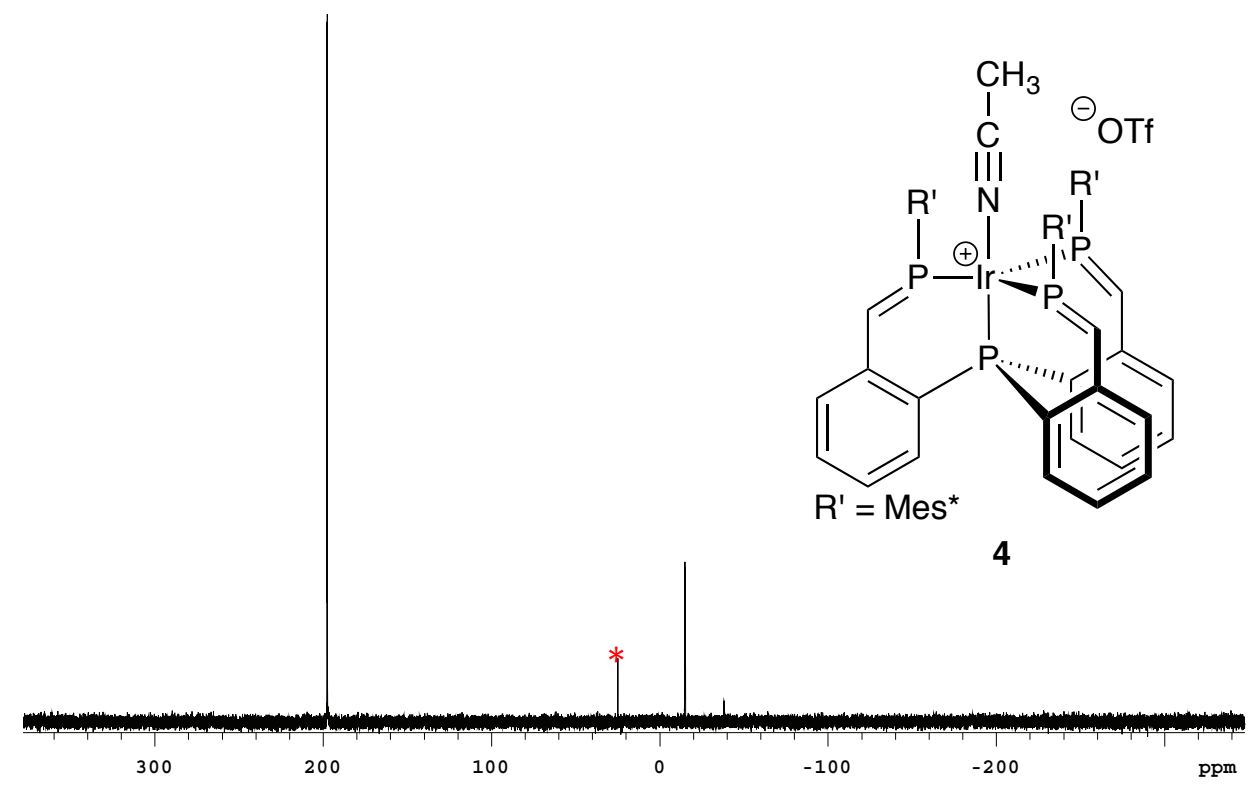

${ }^{31} \mathrm{P}\left\{{ }^{1} \mathrm{H}\right\}$ NMR $\left(\mathrm{C}_{6} \mathrm{D}_{6}\right)$ : Phosphaalkene region

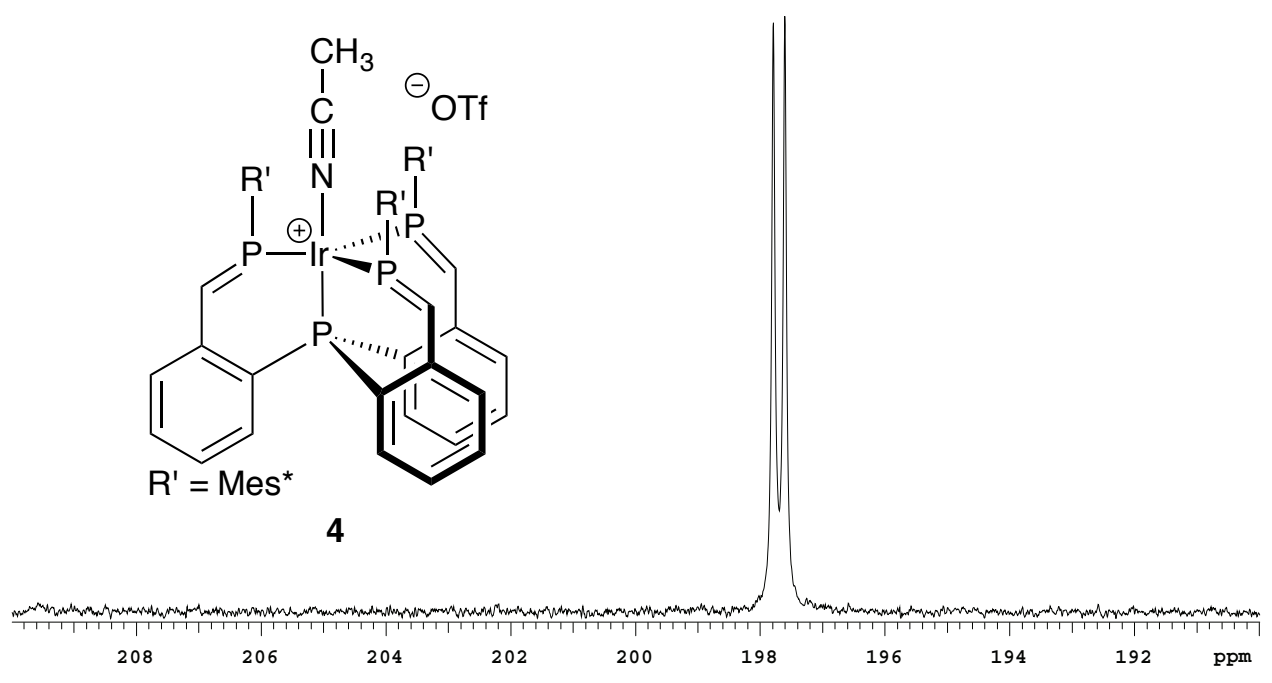


${ }^{31} \mathrm{P}\left\{{ }^{1} \mathrm{H}\right\}$ NMR $\left(\mathrm{C}_{6} \mathrm{D}_{6}\right):$ Apical P

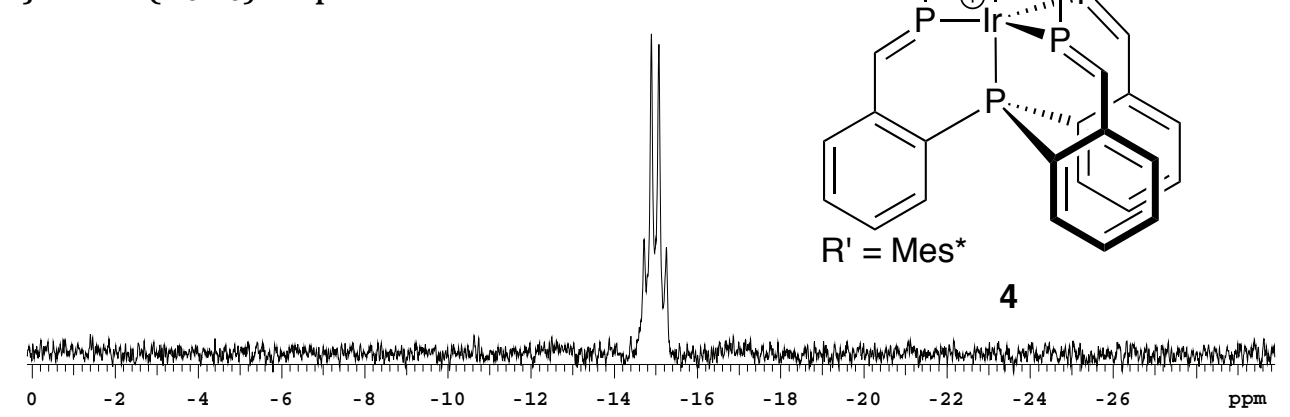

${ }^{1} \mathrm{H}$ NMR $\left(\mathrm{C}_{6} \mathrm{D}_{6}\right)$
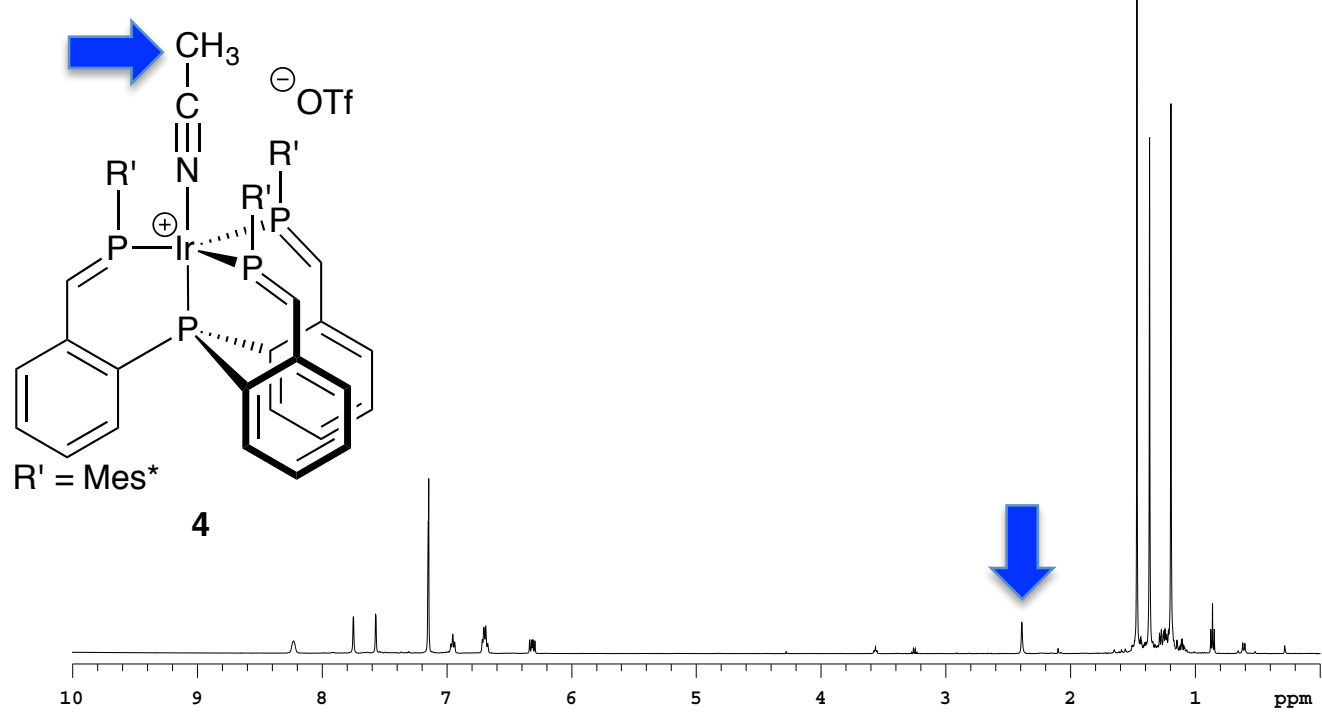
${ }^{1} \mathrm{H}$ NMR $\left(\mathrm{C}_{6} \mathrm{D}_{6}\right)$ : Aryl region. Note two separate Mes* resonances (singlets) suggesting the presence of restricted rotation about P-Mes* group. The large singlet is benzene.

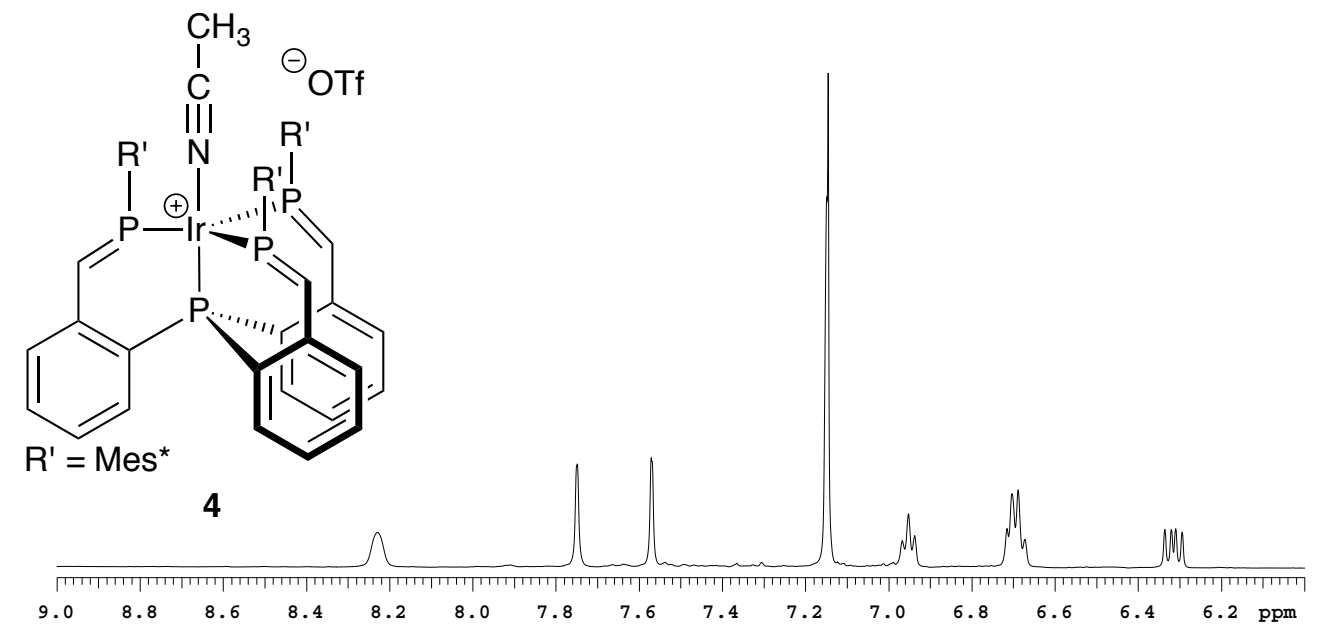

${ }^{13} \mathrm{C}\left\{{ }^{1} \mathrm{H}\right\}$ NMR $\left(\mathrm{C}_{6} \mathrm{D}_{6}\right)$ : Note three separate $t$-Bu signals suggesting the presence of restricted rotation about $\mathrm{P}-\mathrm{Mes}$ * group.

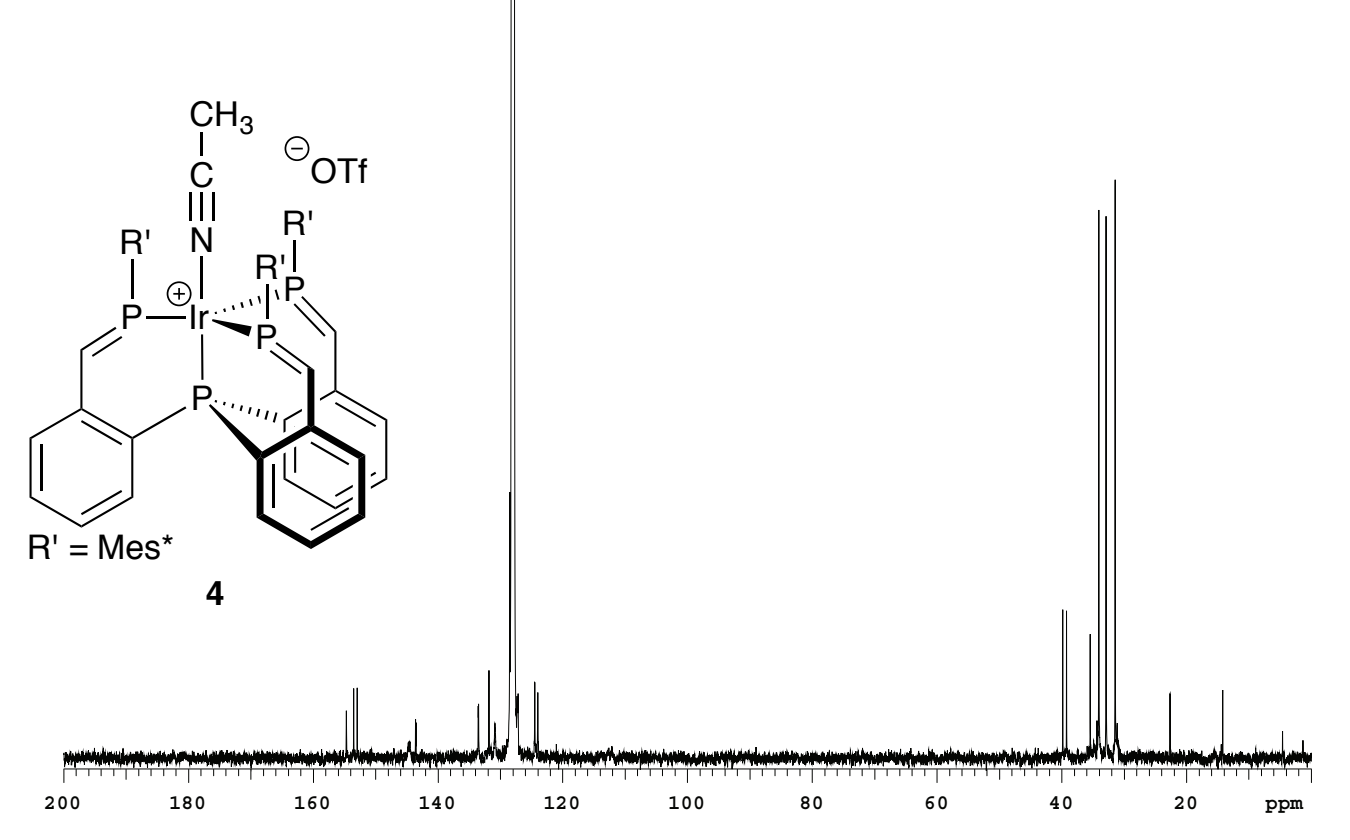


${ }^{13} \mathrm{C}\left\{{ }^{1} \mathrm{H}\right\}$ NMR $\left(\mathrm{C}_{6} \mathrm{D}_{6}\right)$ : Alkyl region. Note three separate $t$-Bu signals suggesting the presence of restricted rotation about P-Mes* group. Pentane is labeled with red asterisks $(*)$.

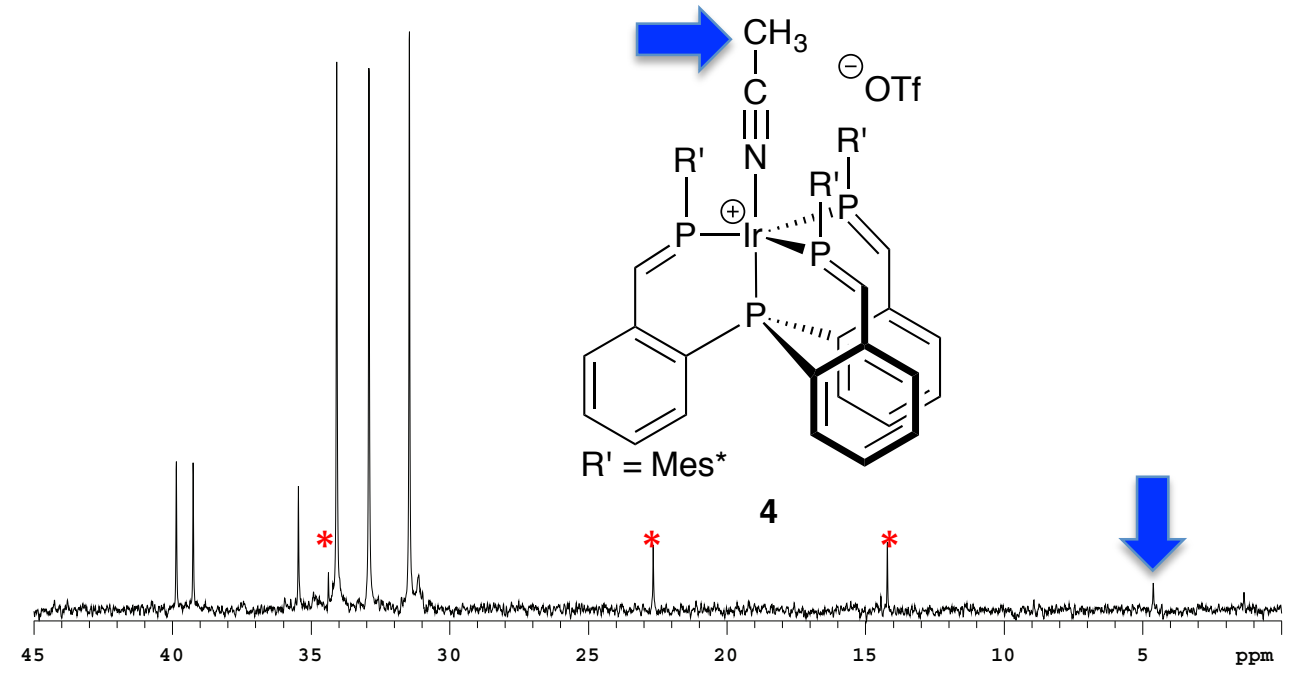

${ }^{13} \mathrm{C}-{ }^{1} \mathrm{H}$ HSQC $\left(\mathrm{C}_{6} \mathrm{D}_{6}\right)$ : The cross peak of interest is highlighted in the blue box.

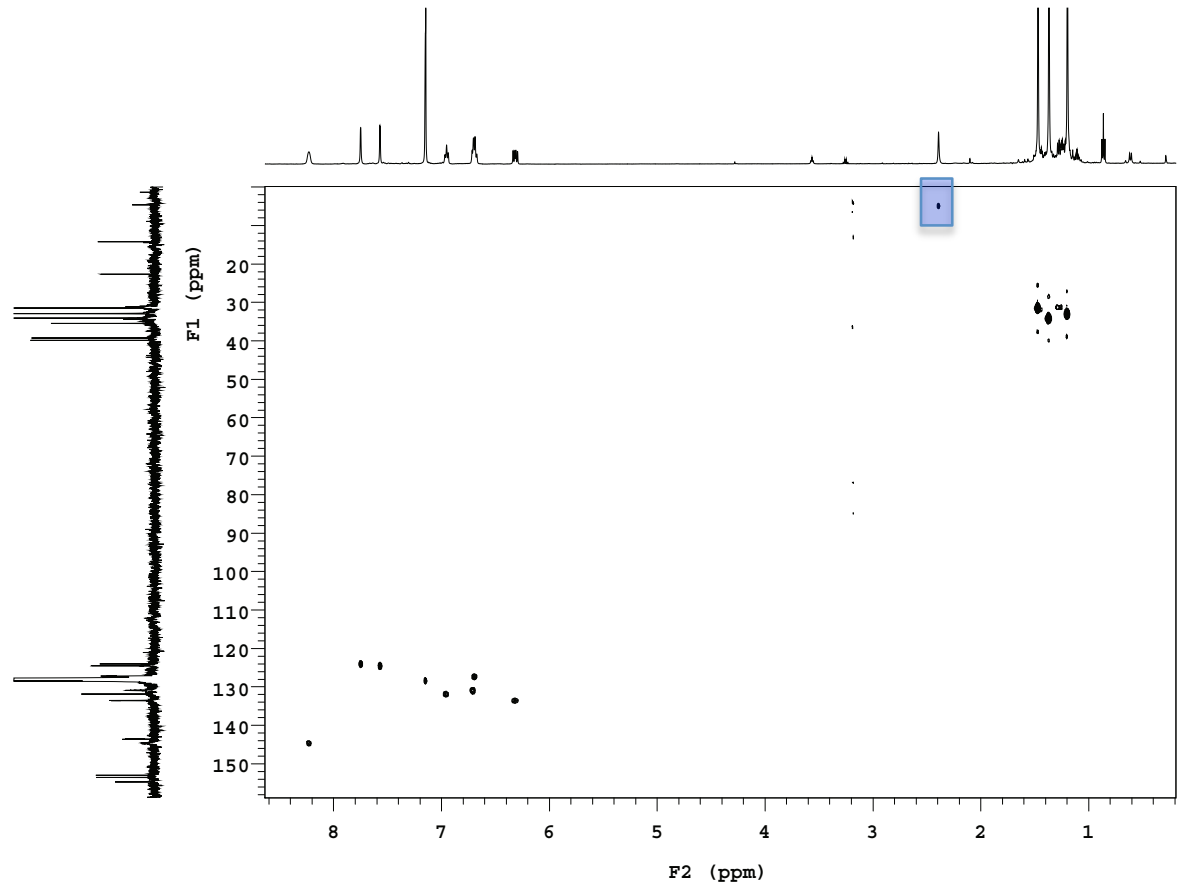


${ }^{13} \mathrm{C}-{ }^{1} \mathrm{H}$ HSQC $\left(\mathrm{C}_{6} \mathrm{D}_{6}\right)$ : Alkyl region. The cross peak of interest is highlighted in the blue box.

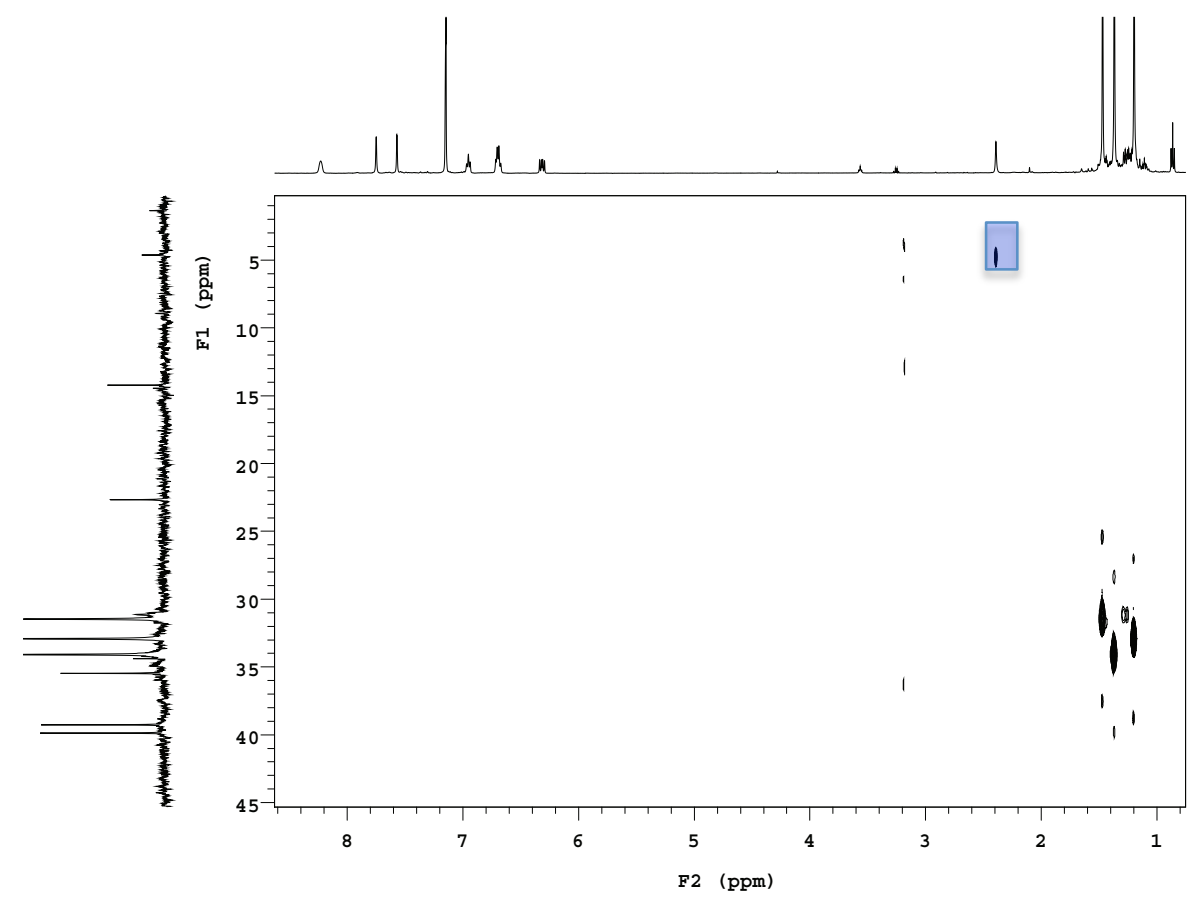

${ }^{13} \mathrm{C}-{ }^{1} \mathrm{H}$ HSQC $\left(\mathrm{C}_{6} \mathrm{D}_{6}\right)$ : Extreme zoom in of acetonitrile cross peak.
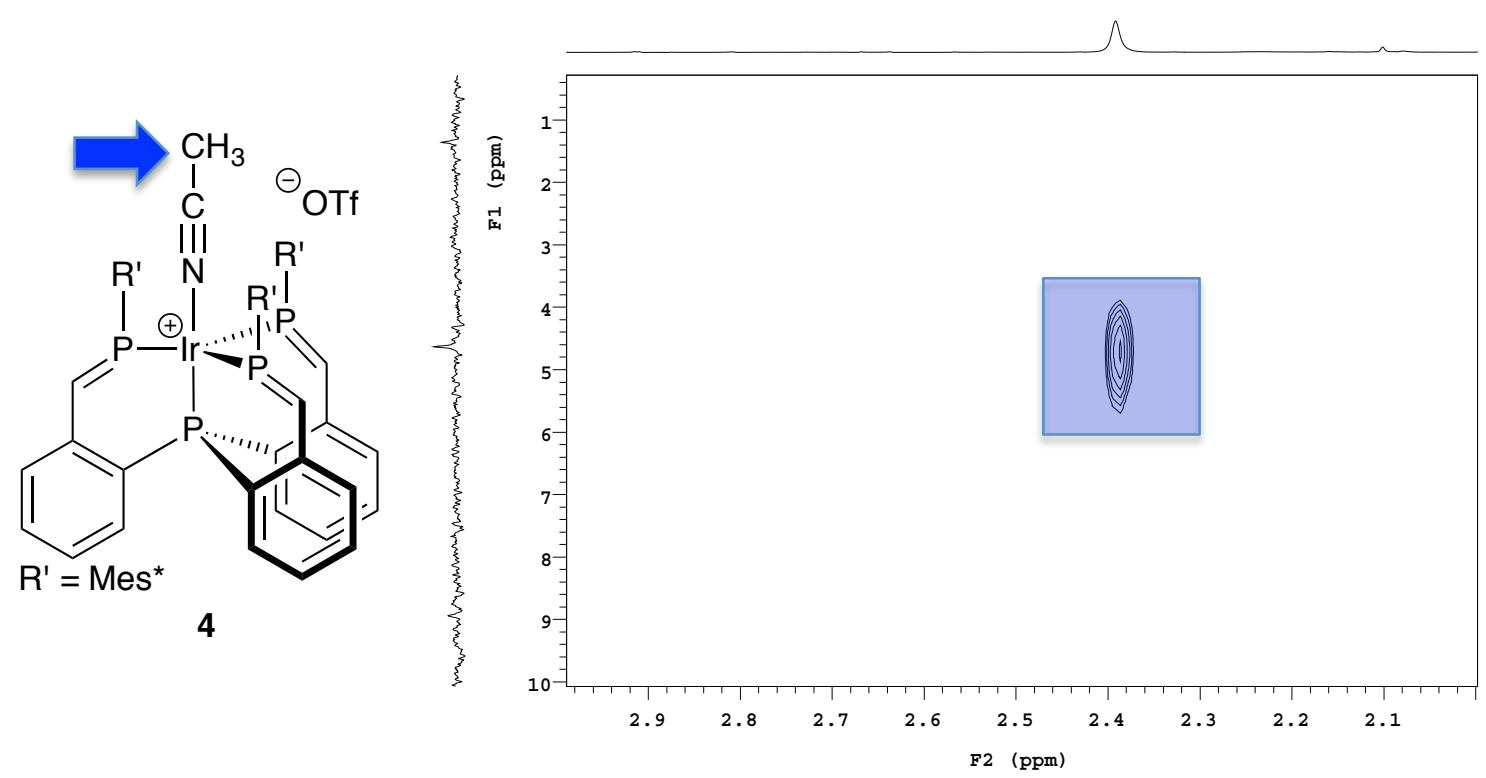
${ }^{1} \mathrm{H}$ NMR $\left(\mathrm{C}_{6} \mathrm{D}_{6}\right) . \mathrm{CD}_{3} \mathrm{CN}$ analogue. No peak at $2.39 \mathrm{ppm}$.

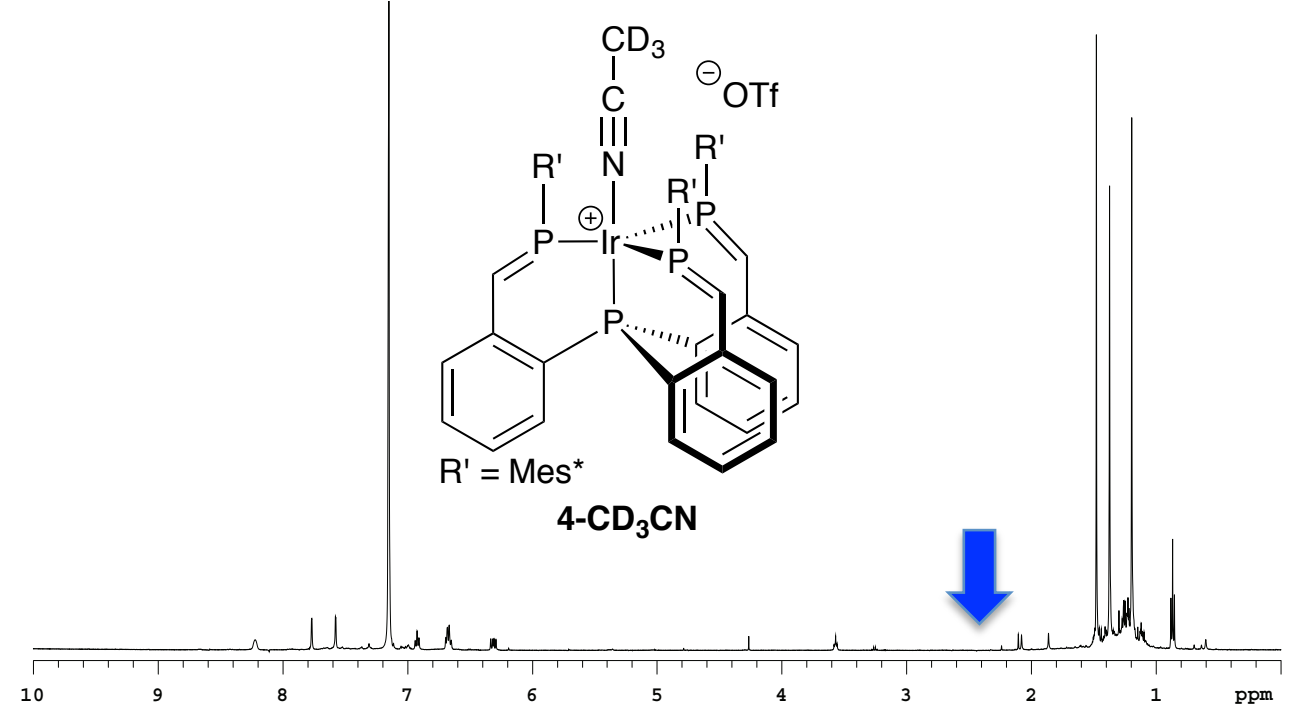

${ }^{19} \mathrm{~F}\left\{{ }^{1} \mathrm{H}\right\} \operatorname{NMR}\left(\mathrm{C}_{6} \mathrm{D}_{6}\right)$

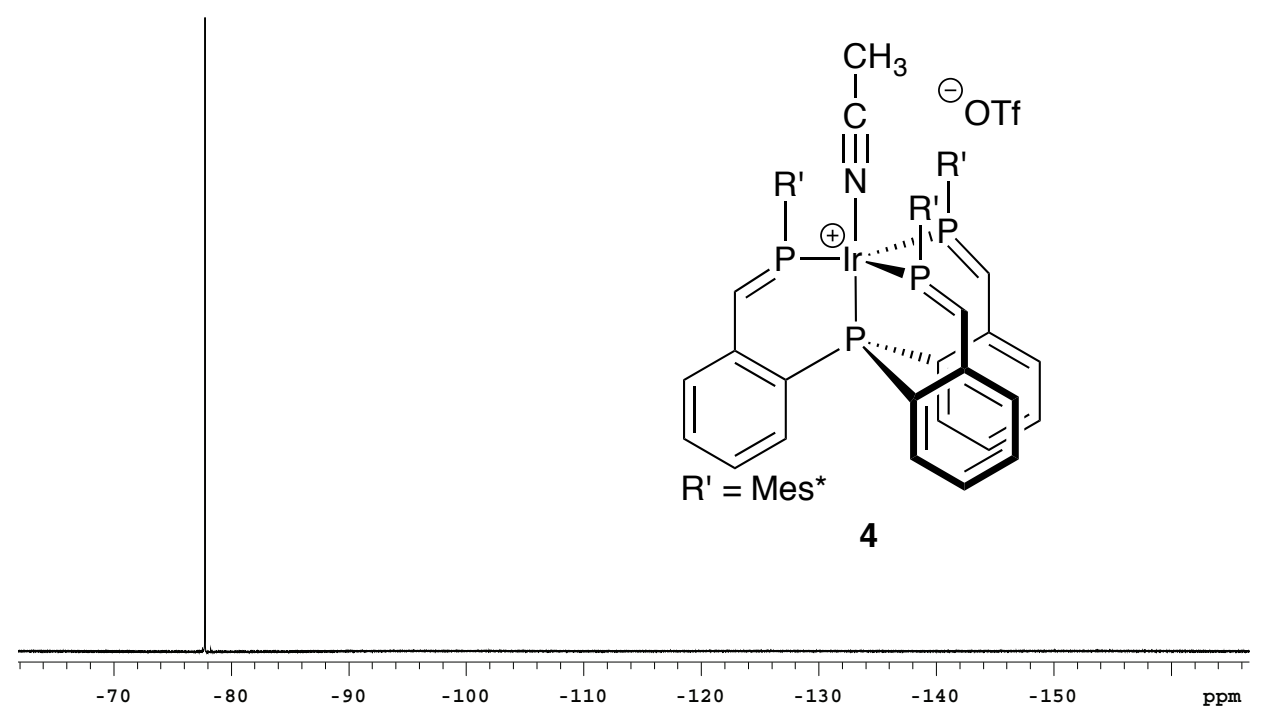




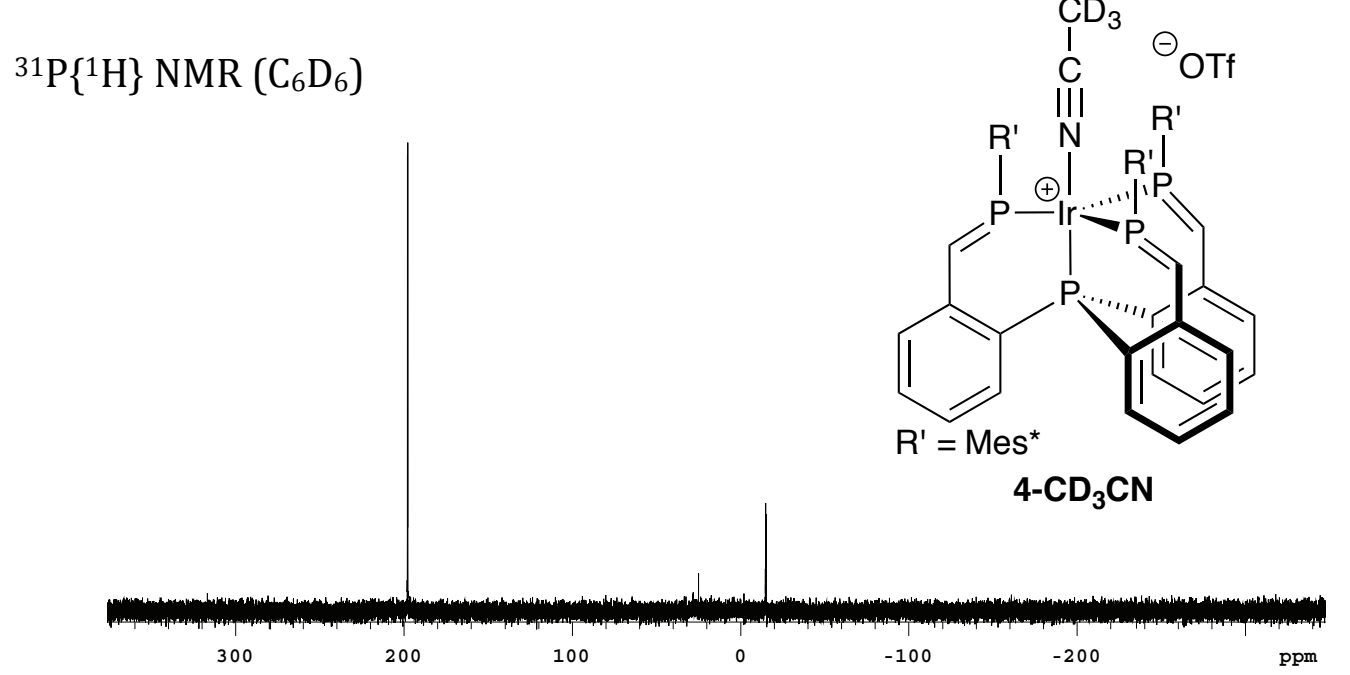

${ }^{31} \mathrm{P}\left\{{ }^{1} \mathrm{H}\right\} \mathrm{NMR}\left(\mathrm{C}_{6} \mathrm{D}_{6}\right)$ : Phosphaalkene region

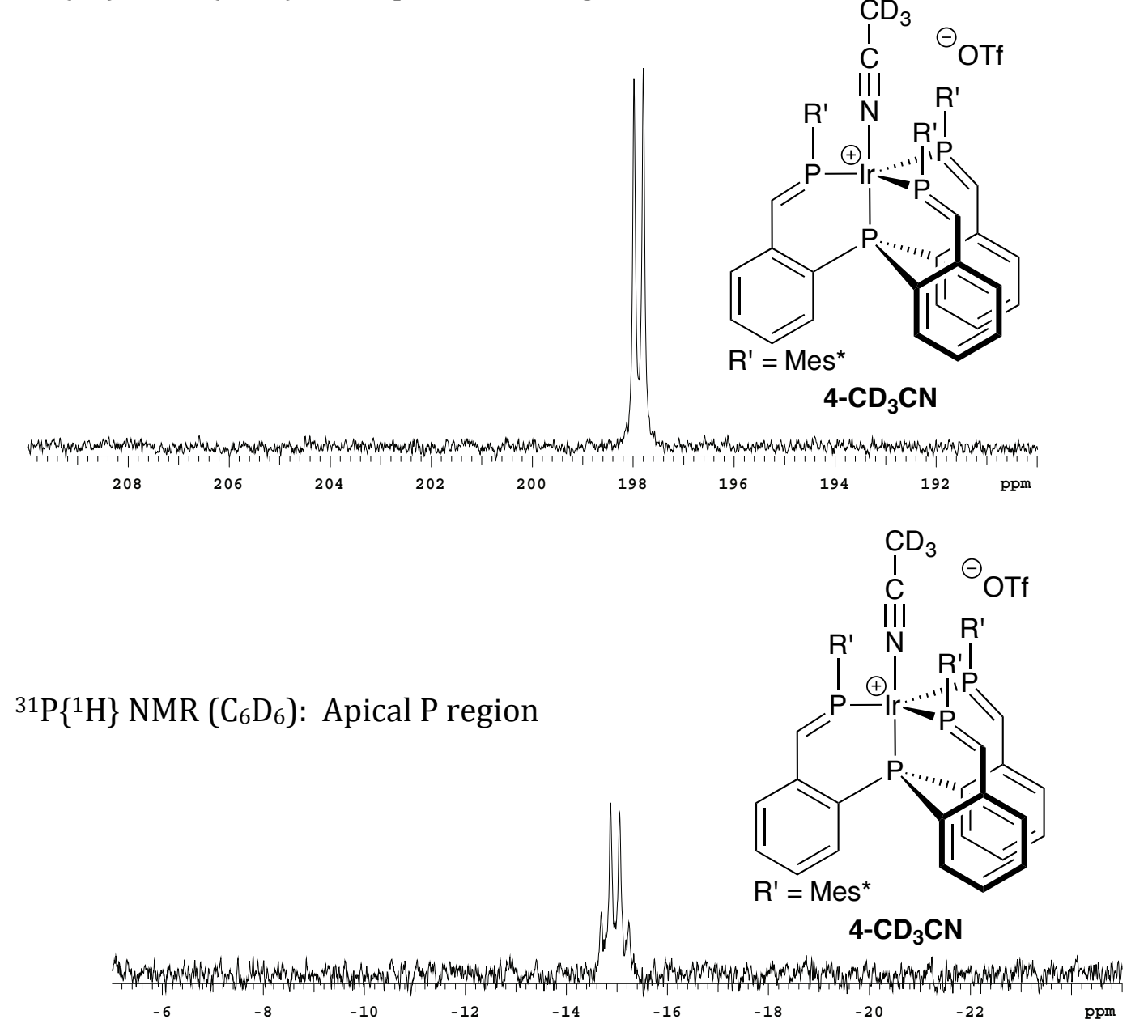


${ }^{1} \mathrm{H}$ NMR $\left(\mathrm{C}_{6} \mathrm{D}_{6}\right)$ : Same NMR is shown above right after $2 \mathrm{D}$ data

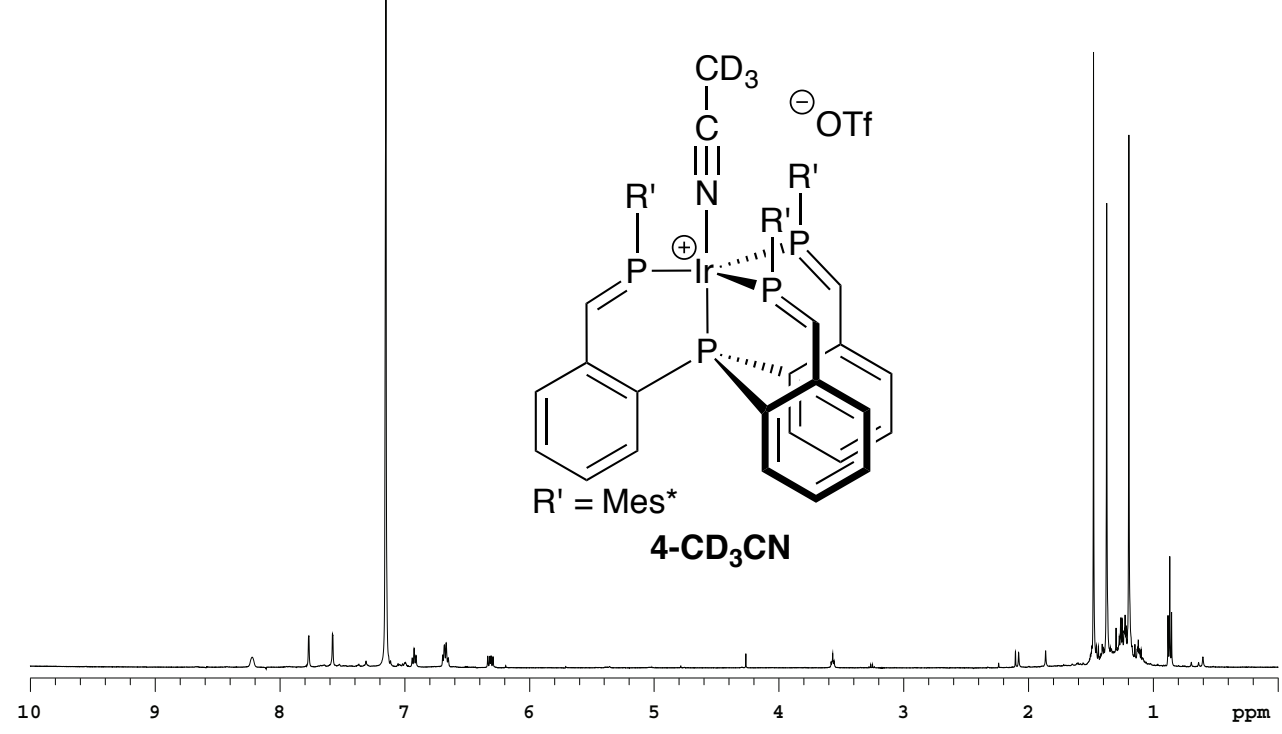




\section{DFT Computational Details}

Full-molecule calculations were carried out using the hybrid M06 functional ${ }^{1}$ and the LACV3P** basis, $^{2}$ as implemented in the Jaguar $^{3}$ suite of programs. Natural Bond Orbital calculations ${ }^{4}$ were performed on the optimized structure using NBO $6.0,{ }^{5}$ as implemented in Jaguar. Computed structures were confirmed as minima by calculating the vibrational frequencies using second derivative analytic methods, and confirming the absence of imaginary frequencies for minima. Thermodynamic quantities were calculated assuming an ideal gas, and are zero point energy corrected. Graphical representations of structures were made using the CYLView program. ${ }^{6}$

\section{Material Relevant to all DFT output}

Jaguar version 8.6, release 13, Schrodinger, LLC, New York, NY, 2014

basis set: lacv3p**

net molecular charge: 0

multiplicity: 1

Non-default options chosen:

SCF calculation type: DFT (m06)

DFT $=$ M06

Vibrational frequencies and related properties computed from analytic second

derivatives

Molecular symmetry not used

Energy convergence criterion: $1.00 \mathrm{E}-05$ hartrees

RMS density matrix convergence criterion: $1.00 \mathrm{E}-06$

Highest accuracy cutoffs used in SCF

\section{References.}

(1) (a) Zhao, Y.; Truhlar, D. G. Theor. Chem. Acc. 2008, 120, 215-241; (b) Zhao, Y.; Truhlar, D. G. Acc. Chem. Res. 2008, 41, 157-167.

(2) (a) Dunning, T. H.; Hay, P. J., In Modern Theoretical Chemistry, Vol. 4: Applications of Electronic Structure Theory. Schaefer, H. F., III (Editor); Plenum, NY: 1977 ; (b) Wadt, W. R.; Hay, P. J. J. Chem. Phys. 1985, 82, 284-298; (c) Hay, P. J.; Wadt, W. R. J. Chem. Phys. 1985, 82, 299-310; (d) Hay, P. J.; Wadt, W. R. J. Chem. Phys. 1985, 82, 270-283.

(3) (a) Bochevarov, A. D.; Harder, E.; Hughes, T. F.; Greenwood, J. R.; Braden, D. A.; Philipp, D. M.; Rinaldo, D.; Halls, M. D.; Zhang, J.; Friesner, R. A. Int. J. Quantum Chem. 2013, 113, 2110-2142; (b) Jaguar, versions 7.0-8.9, Schrödinger, LLC, New York, NY: 2007-2015.

(4) (a) Weinhold, F.; Landis, C. R., Discovering Chemistry with Natural Bond Orbitals, John Wiley \& Sons: NJ, 2012; (b) Glendening, E. D.; Landis, C. R.; Weinhold, F. Wiley Interdisciplinary Reviews: Computational Molecular Science 2012, 2, 1-42; (c) Weinhold, F.; Landis, C. R., Valency and Bonding: A Natural Bond Orbital Donor-Acceptor Perspective, Cambridge University Press: Cambridge, 2005; (d) Reed, A. E.; Curtiss, L. A.; Weinhold, F. Chem. Rev. 1988, 88, 899-926.

(5) (a) Glendening, E. D.; Landis, C. R.; Weinhold, F. J. Comp. Chem. 2013, 34, 1429-1437; (b) Glendening, E. D.; Badenhoop, J. K.; Reed, A. K.; Carpenter, J. E.; Bohmann, J. A.; Morales, C. M.; Landis, C. R.; Weinhold, F. NBO 6.0, Theoretical Chemistry Institute, University of Wisconsin; Madison: 2013.

(6) Legault, C. Y. CYLview, 1.0b, Université de Sherbrooke: Université de Sherbrooke, 2009.

Note: XYZ files are listed as separate attachments on the manuscript. 


\section{Electrochemical Experimental Details including addition Cyclic Voltammetry Plots}

\section{General Experimental Details:}

All electrochemical measurements were conducted on a CHI 1040 Electrochemical Analyzer with a three-electrode system. A glassy carbon electrode, a platinum wire, and a $\mathrm{Ag} / \mathrm{Ag}^{+}$electrode were employed as the working, auxiliary, and reference electrodes, respectively. The $\mathrm{Fc} / \mathrm{Fc}^{+}$couple was employed as the internal standard. Cyclic voltammetry $(\mathrm{CV})$ was performed in a $0.1 \mathrm{M}$ solution of $\left[\mathrm{Bu}_{4} \mathrm{~N}\right]\left[\mathrm{PF}_{6}\right]$ in anhydrous dichloromethane or tetrahydrofuran at room temperature under a nitrogen atmosphere.

CV plots at varying scan rates are shown below. An overlay plot is also included.

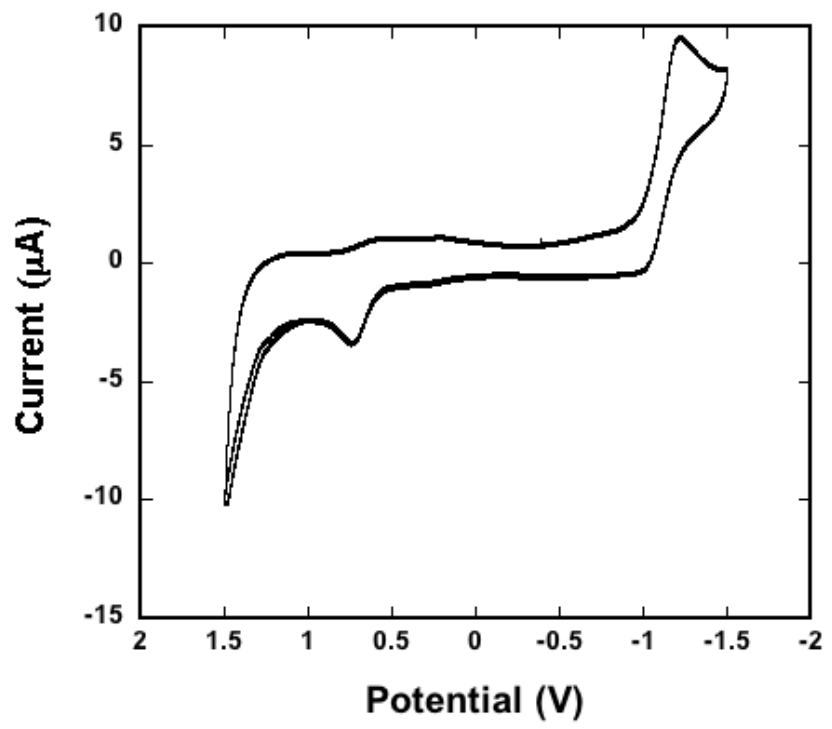

Figure S1. DCM, $50 \mathrm{mV}$ scan rate

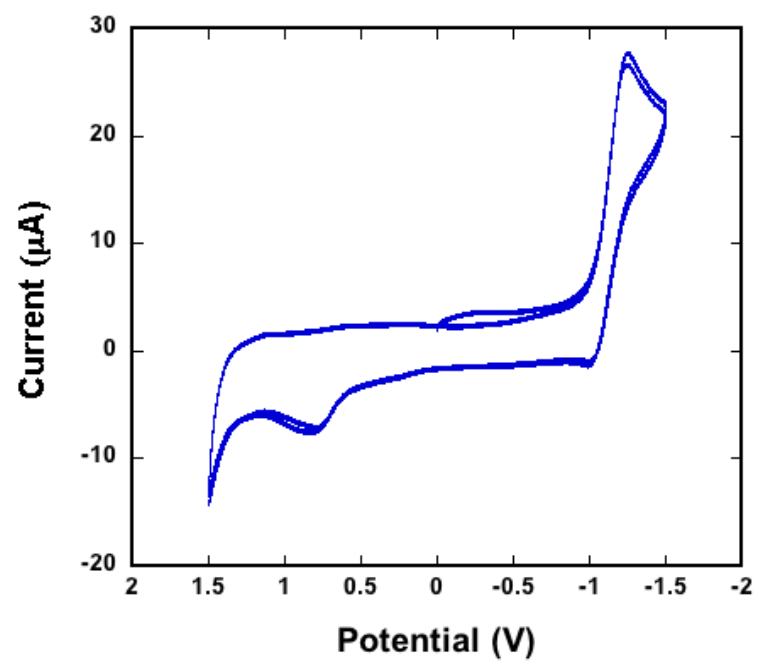

Figure S2. DCM, $100 \mathrm{mV}$ scan Rate 


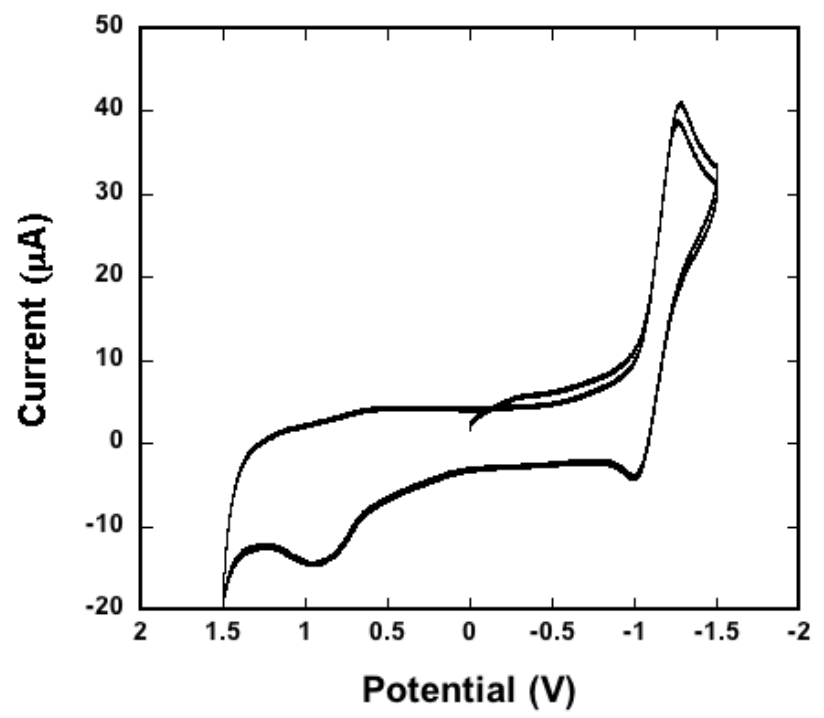

Figure S3. DCM, $200 \mathrm{mV}$ scan rate

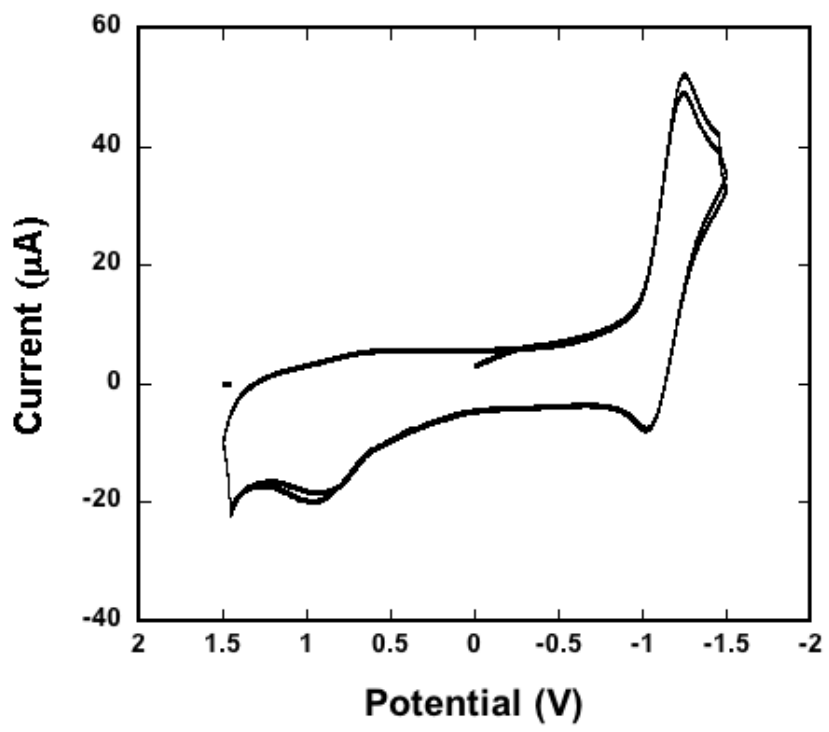

Figure S4. DCM, $300 \mathrm{mV}$ scan rate 


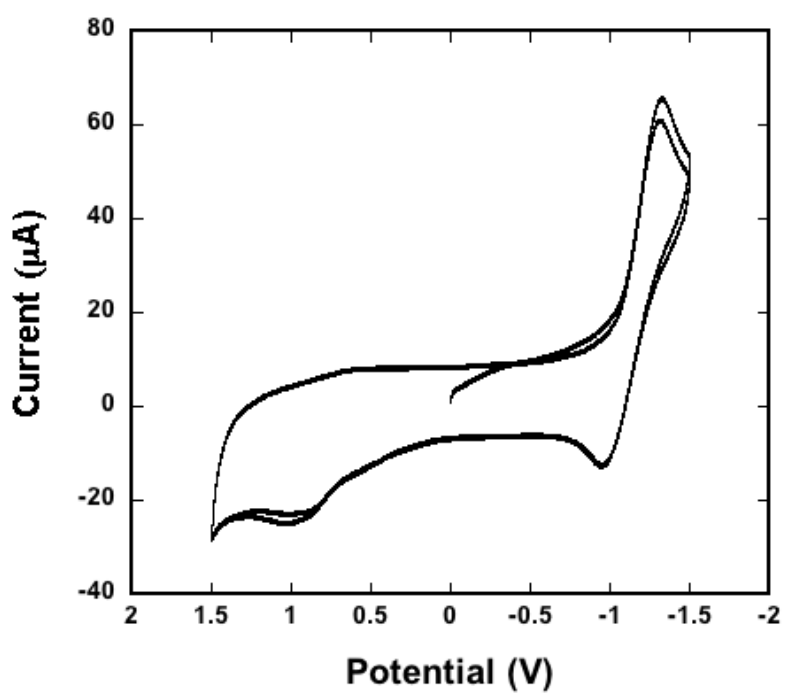

Figure S5. DCM, $500 \mathrm{mV}$ scan rate

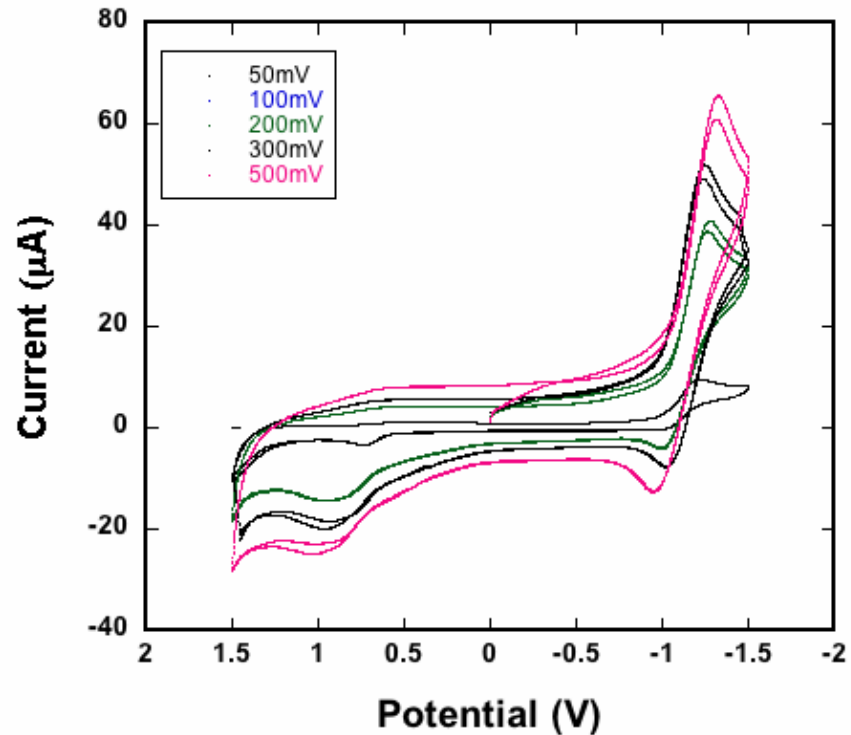

Figure S6. DCM, overlay plot

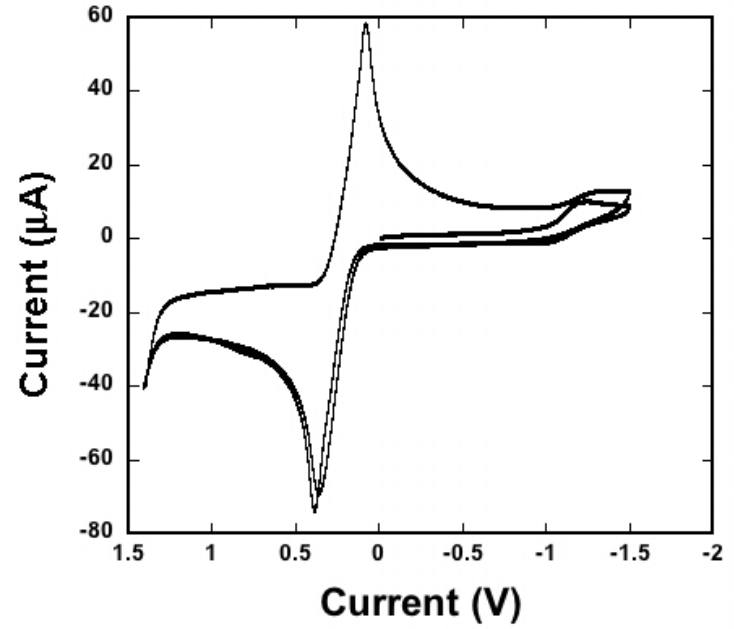

Figure S7. $\mathrm{CV}$ of $1+$ Ferrocene in $\mathrm{CH}_{2} \mathrm{Cl}_{2}\left(0.1 \mathrm{M}\left[\mathrm{Bu}_{4} \mathrm{~N}\right]\left[\mathrm{PF}_{6}\right]\right)$. Scan rate $100 \mathrm{mV} / \mathrm{s}$. Ref: $\mathrm{Ag} / \mathrm{Ag}^{+}$ 


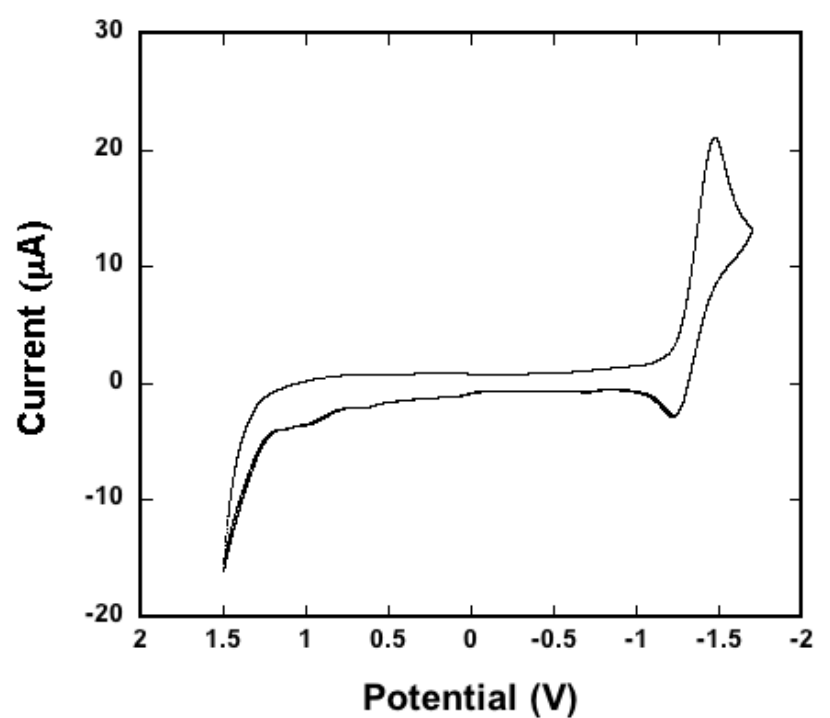

Figure S8. THF, $50 \mathrm{mV}$ scan rate

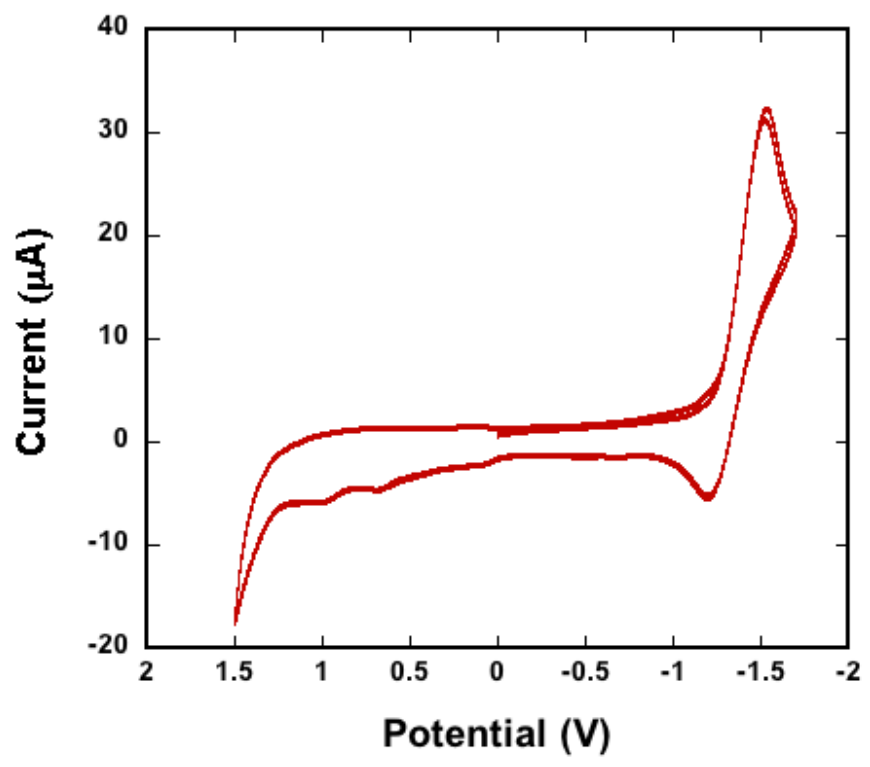

Figure S9. THF, $100 \mathrm{mV}$ scan rate 


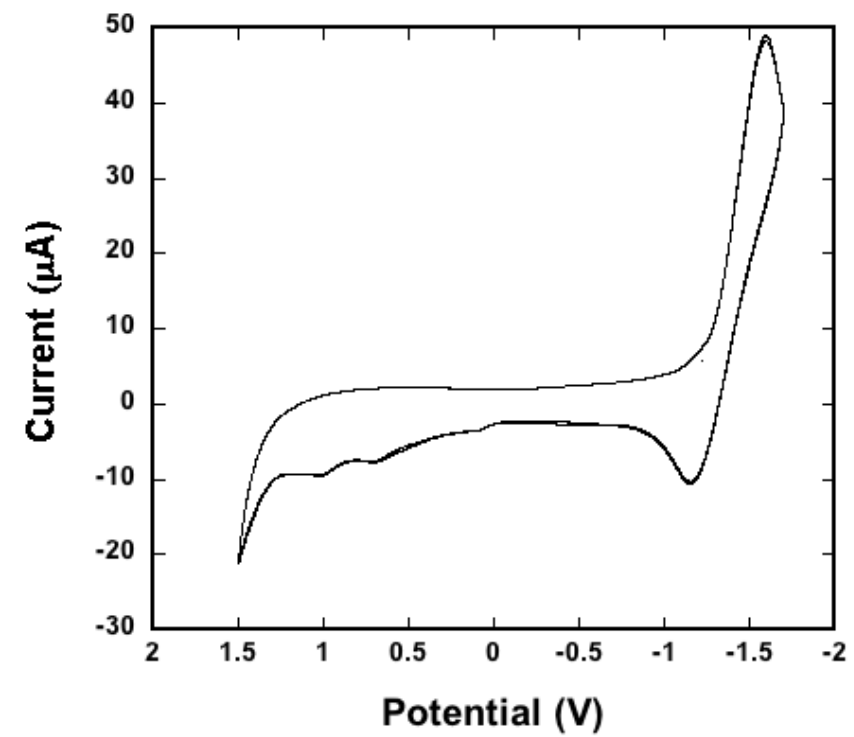

Figure S10. THF, $200 \mathrm{mV}$ scan rate

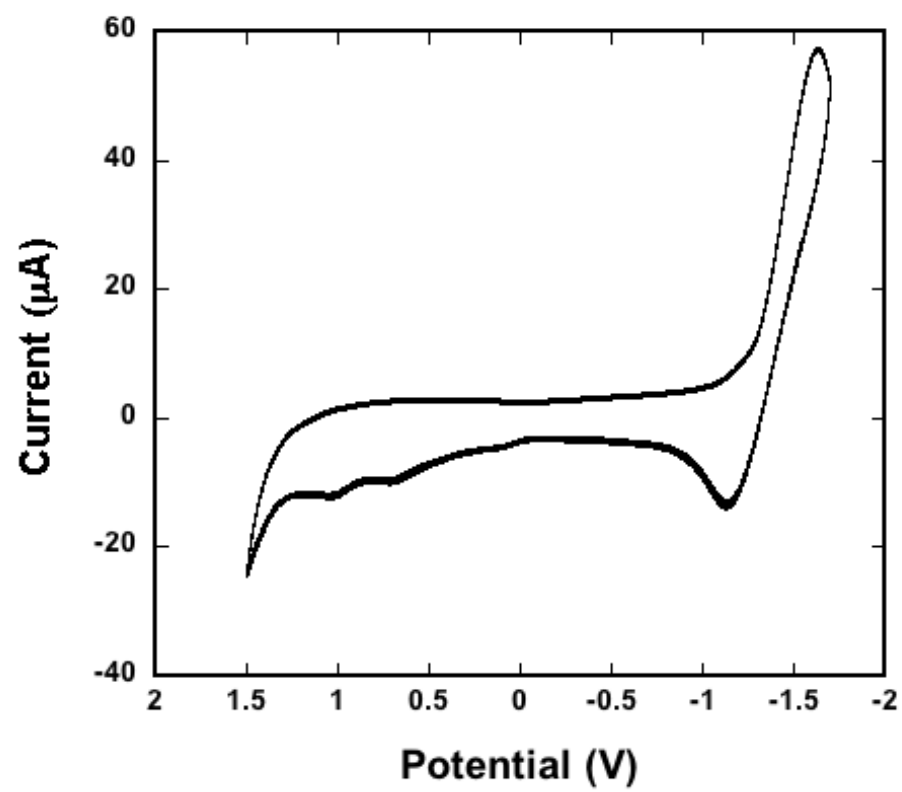

Figure S11. THF, $300 \mathrm{mV}$ scan rate 


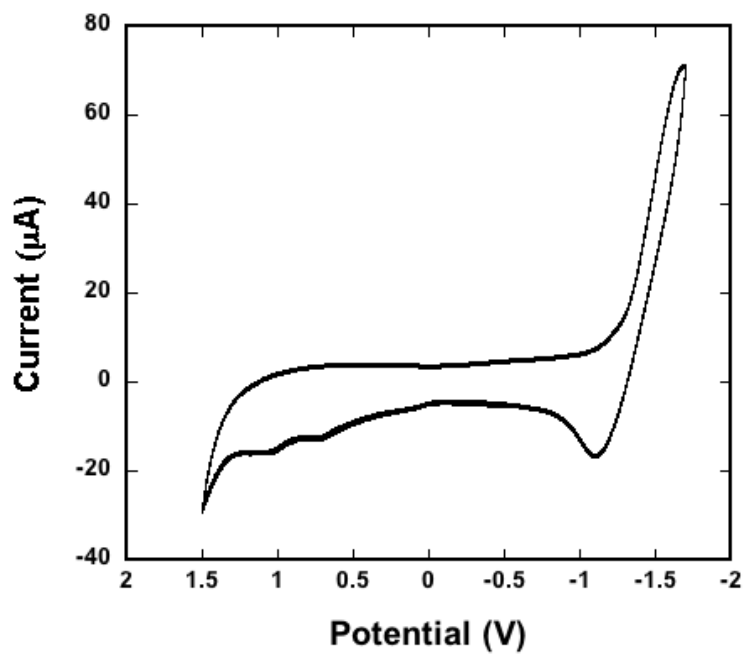

Figure S12. THF, $500 \mathrm{mV}$ scan rate

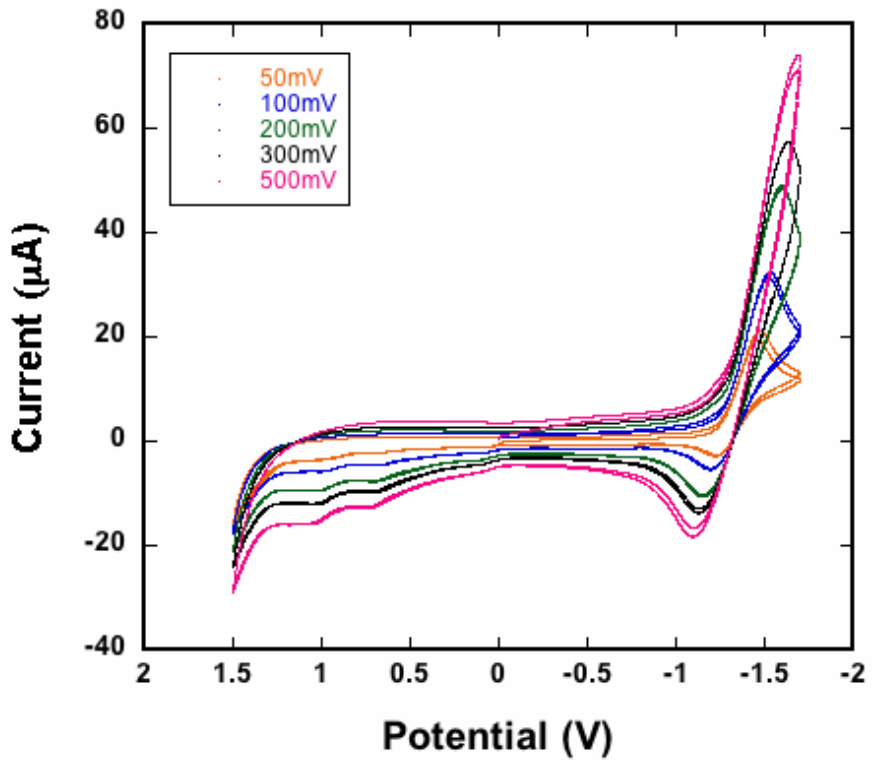

Figure S13. THF, overlay plot 


\section{Experimental Details for Crystal Structure Refinement and Acquisition (including references)}

All samples were coated with Paratone-N oil and mounted on Cryo-Loops. Data were collected at $100 \mathrm{~K}$. For $\mathbf{1}$, data were collected on a Bruker MicroStar using a $\mathrm{Cu}$ rotatinganode source and micro-focus optics. For $\mathbf{2} \mathbf{a}$ and $\mathbf{2 b}$ data were collected on a Bruker Ultra using a Mo rotating anode source and micro-focus optics. Data were processed and corrections made using the standard methods contained in the Bruker ApexII software. All subsequent refinement and documentation used the OLEX2 platform of programs. In all three cases, considerable solvent disorder required the implementation of the Platon subroutine, SQUEEZE, which renders void-space occupancy as a diffuse contribution. Based on the found electron count, the approximate empirical formula for 1 is $\mathbf{1} \cdot 1.5 \mathrm{THF}$, for $\mathbf{2 a}$ is $\mathbf{2} \mathbf{a} \cdot 5 \mathrm{CHCl}_{3}$, and for $\mathbf{2 b}$ is $\mathbf{2} \mathbf{b} \cdot 2.5 \mathrm{CHCl}_{3}$.

\section{Data for 1}

Table S1. Crystal data and structure refinement for cain01_sq (1).

Identification code

Empirical formula

Formula weight

Temperature

Wavelength

Crystal system

Space group

Unit cell dimensions

Volume

$\mathrm{Z}$

Density (calculated)

Absorption coefficient

$\mathrm{F}(000)$

Crystal size

Theta range for data collection

Index ranges

Reflections collected

Independent reflections

Completeness to theta $=66.000^{\circ}$ cain01_sq

C75 H102 P4

1127.44

$100.0 \mathrm{~K}$

$1.54178 \AA$

Monoclinic

P 1 21/n 1

$\mathrm{a}=13.5848(5) \AA \quad \alpha=90^{\circ}$.

$\mathrm{b}=24.3520(8) \AA \quad \beta=93.536(2)^{\circ}$.

$\mathrm{c}=23.4755(8) \AA \quad \gamma=90^{\circ}$.

$7751.3(5) \AA^{3}$

4

$0.966 \mathrm{Mg} / \mathrm{m}^{3}$

$1.152 \mathrm{~mm}^{-1}$

2448

$0.29 \times 0.26 \times 0.2 \mathrm{~mm}^{3}$

2.617 to $68.727^{\circ}$.

$-16<=\mathrm{h}<=16,-28<=\mathrm{k}<=29,-28<=\mathrm{l}<=26$

58604

$14080[\mathrm{R}($ int $)=0.0342]$

$99.2 \%$ 

Absorption correction
Max. and min. transmission
Refinement method
Data / restraints / parameters
Goodness-of-fit on $\mathrm{F}^{2}$
Final $\mathrm{R}$ indices [I $>2 \operatorname{sigma}(\mathrm{I})]$
$\mathrm{R}$ indices (all data)
Extinction coefficient
Largest diff. peak and hole
SQUEEZE

Semi-empirical from equivalents

0.1665 and 0.0691

Full-matrix least-squares on $\mathrm{F}^{2}$

14080 / 0 / 764

1.029

$\mathrm{R} 1=0.0550, \mathrm{wR} 2=0.1480$

$\mathrm{R} 1=0.0609, \mathrm{wR} 2=0.1526$

$\mathrm{n} / \mathrm{a}$

0.525 and -0.693 e. $\AA^{-3}$

186e/uc; calc'ed for $6 \mathrm{THF}, 240 \mathrm{e} / \mathrm{uc}$ 
Table S2. Atomic coordinates ( x 10 $0^{4}$ ) and equivalent isotropic displacement parameters $\left(\AA^{2} \times 10^{3}\right)$ for cain01_sq. U(eq) is defined as one third of the trace of the orthogonalized $\mathrm{U}^{\mathrm{ij}}$ tensor.

\begin{tabular}{|c|c|c|c|c|}
\hline & $\mathrm{x}$ & $\mathrm{y}$ & $\mathrm{z}$ & $\mathrm{U}(\mathrm{eq})$ \\
\hline $\mathrm{P}(1)$ & $6561(1)$ & 1990(1) & $4056(1)$ & $35(1)$ \\
\hline $\mathrm{P}(2)$ & $10027(1)$ & 2094(1) & $4107(1)$ & $46(1)$ \\
\hline $\mathrm{P}(3)$ & $5073(1)$ & $1750(1)$ & $2240(1)$ & $44(1)$ \\
\hline $\mathrm{P}(4)$ & $5368(1)$ & $3371(1)$ & $5160(1)$ & $55(1)$ \\
\hline $\mathrm{C}(1)$ & $8821(2)$ & 2164(1) & $4208(1)$ & $40(1)$ \\
\hline$C(2)$ & $8356(2)$ & 1971(1) & $4719(1)$ & $38(1)$ \\
\hline$C(3)$ & $7332(2)$ & $1870(1)$ & $4715(1)$ & $38(1)$ \\
\hline $\mathrm{C}(4)$ & $6927(2)$ & $1696(1)$ & $5220(1)$ & $45(1)$ \\
\hline$C(5)$ & $7510(2)$ & 1621(1) & $5714(1)$ & $51(1)$ \\
\hline$C(6)$ & $8512(2)$ & 1721(1) & 5723(1) & $50(1)$ \\
\hline $\mathrm{C}(7)$ & $8926(2)$ & 1891(1) & $5231(1)$ & $45(1)$ \\
\hline $\mathrm{C}(8)$ & $10119(2)$ & 2472(1) & $3428(1)$ & $42(1)$ \\
\hline $\mathrm{C}(9)$ & $9976(2)$ & 2196(1) & $2896(1)$ & $43(1)$ \\
\hline $\mathrm{C}(10)$ & $9856(2)$ & $2515(1)$ & 2404(1) & $44(1)$ \\
\hline$C(11)$ & $9879(2)$ & $3083(1)$ & $2407(1)$ & $43(1)$ \\
\hline $\mathrm{C}(12)$ & $10106(2)$ & $3339(1)$ & $2928(1)$ & $44(1)$ \\
\hline$C(13)$ & $10266(2)$ & $3051(1)$ & $3437(1)$ & $42(1)$ \\
\hline $\mathrm{C}(14)$ & $9967(2)$ & 1561(1) & $2827(1)$ & $50(1)$ \\
\hline$C(15)$ & 10851(2) & 1299(1) & $3161(1)$ & $64(1)$ \\
\hline$C(16)$ & $8992(2)$ & 1311(1) & $3008(1)$ & $64(1)$ \\
\hline$C(17)$ & $10051(2)$ & 1389(1) & 2197(1) & $60(1)$ \\
\hline$C(18)$ & $9669(2)$ & $3430(1)$ & $1866(1)$ & $50(1)$ \\
\hline$C(19)$ & $8641(2)$ & $3686(1)$ & $1900(1)$ & $59(1)$ \\
\hline $\mathrm{C}(20)$ & $10423(2)$ & $3897(1)$ & $1830(1)$ & $69(1)$ \\
\hline $\mathrm{C}(21)$ & $9689(2)$ & $3085(1)$ & $1320(1)$ & $61(1)$ \\
\hline $\mathrm{C}(22)$ & $10657(2)$ & $3384(1)$ & $3974(1)$ & $45(1)$ \\
\hline$C(23)$ & $11566(2)$ & $3096(1)$ & $4266(1)$ & $54(1)$ \\
\hline $\mathrm{C}(24)$ & $11010(2)$ & $3957(1)$ & $3811(1)$ & $53(1)$ \\
\hline$C(25)$ & $9865(2)$ & $3466(1)$ & $4407(1)$ & $51(1)$ \\
\hline$C(26)$ & $5904(2)$ & $1777(1)$ & 2798(1) & $39(1)$ \\
\hline$C(27)$ & $6296(2)$ & $1295(1)$ & $3106(1)$ & $40(1)$ \\
\hline
\end{tabular}




\begin{tabular}{|c|c|c|c|c|}
\hline $\mathrm{C}(28)$ & $6652(2)$ & $1328(1)$ & $3684(1)$ & $39(1)$ \\
\hline$C(29)$ & $7035(2)$ & $861(1)$ & $3958(1)$ & $49(1)$ \\
\hline $\mathrm{C}(30)$ & $7048(2)$ & $360(1)$ & $3679(1)$ & $60(1)$ \\
\hline $\mathrm{C}(31)$ & $6691(2)$ & $324(1)$ & $3116(1)$ & $59(1)$ \\
\hline$C(32)$ & $6332(2)$ & 783(1) & 2832(1) & $49(1)$ \\
\hline$C(33)$ & $5014(2)$ & 2484(1) & 2041(1) & $39(1)$ \\
\hline$C(34)$ & $4304(2)$ & 2833(1) & $2278(1)$ & $40(1)$ \\
\hline$C(35)$ & $4430(2)$ & $3397(1)$ & 2232(1) & $44(1)$ \\
\hline$C(36)$ & $5187(2)$ & $3636(1)$ & 1954(1) & $45(1)$ \\
\hline $\mathrm{C}(37)$ & $5803(2)$ & $3288(1)$ & $1673(1)$ & $45(1)$ \\
\hline $\mathrm{C}(38)$ & $5729(2)$ & 2715(1) & $1695(1)$ & $40(1)$ \\
\hline C(39) & $3365(2)$ & 2621(1) & $2553(1)$ & $45(1)$ \\
\hline$C(40)$ & $2829(2)$ & 2203(1) & 2152(1) & $58(1)$ \\
\hline$C(41)$ & $3596(2)$ & $2366(1)$ & $3145(1)$ & $52(1)$ \\
\hline$C(42)$ & $2624(2)$ & 3081(1) & 2632(1) & $55(1)$ \\
\hline$C(43)$ & $5299(2)$ & $4262(1)$ & 1927(1) & $58(1)$ \\
\hline $\mathrm{C}(44)$ & $4430(6)$ & 4492(2) & $1502(3)$ & $74(2)$ \\
\hline$C\left(44^{\prime}\right)$ & $4395(5)$ & $4546(2)$ & 2061(4) & $96(3)$ \\
\hline $\mathrm{C}(45)$ & $5124(5)$ & $4544(2)$ & $2505(2)$ & $61(2)$ \\
\hline$C\left(45^{\prime}\right)$ & $6165(4)$ & $4388(2)$ & 2385(3) & $72(2)$ \\
\hline $\mathrm{C}(46)$ & $6241(6)$ & $4464(3)$ & $1705(4)$ & $85(3)$ \\
\hline$C\left(46^{\prime}\right)$ & $5639(8)$ & $4424(3)$ & 1343(3) & $107(3)$ \\
\hline $\mathrm{C}(47)$ & $6400(2)$ & $2370(1)$ & $1318(1)$ & $45(1)$ \\
\hline $\mathrm{C}(48)$ & $6839(2)$ & $2726(1)$ & $856(1)$ & $51(1)$ \\
\hline $\mathrm{C}(49)$ & $7279(2)$ & 2111(1) & $1666(1)$ & $48(1)$ \\
\hline $\mathrm{C}(50)$ & $5797(2)$ & 1922(1) & 991(1) & $53(1)$ \\
\hline $\mathrm{C}(51)$ & $5466(2)$ & 2919(1) & $4627(1)$ & $40(1)$ \\
\hline $\mathrm{C}(52)$ & 4912(2) & 2403(1) & $4574(1)$ & $39(1)$ \\
\hline$C(53)$ & $5312(2)$ & $1936(1)$ & 4311(1) & $38(1)$ \\
\hline$C(54)$ & $4742(2)$ & 1461(1) & $4249(1)$ & $44(1)$ \\
\hline $\mathrm{C}(55)$ & $3800(2)$ & $1438(1)$ & $4439(1)$ & $50(1)$ \\
\hline$C(56)$ & $3409(2)$ & 1892(1) & 4701(1) & $50(1)$ \\
\hline$C(57)$ & $3961(2)$ & $2364(1)$ & $4766(1)$ & $45(1)$ \\
\hline $\mathrm{C}(58)$ & $6072(2)$ & $3957(1)$ & $4879(1)$ & $43(1)$ \\
\hline $\mathrm{C}(59)$ & $7062(2)$ & $4054(1)$ & $5083(1)$ & $40(1)$ \\
\hline $\mathrm{C}(60)$ & $7627(2)$ & $4415(1)$ & 4784(1) & $41(1)$ \\
\hline
\end{tabular}




\begin{tabular}{lrrrr}
$\mathrm{C}(61)$ & $7269(2)$ & $4689(1)$ & $4301(1)$ & $50(1)$ \\
$\mathrm{C}(62)$ & $6273(2)$ & $4620(1)$ & $4142(1)$ & $56(1)$ \\
$\mathrm{C}(63)$ & $5651(2)$ & $4278(1)$ & $4430(1)$ & $51(1)$ \\
$\mathrm{C}(64)$ & $7555(2)$ & $3779(1)$ & $5625(1)$ & $45(1)$ \\
$\mathrm{C}(65)$ & $6892(2)$ & $3815(1)$ & $6130(1)$ & $58(1)$ \\
$\mathrm{C}(66)$ & $7820(2)$ & $3178(1)$ & $5504(1)$ & $52(1)$ \\
$\mathrm{C}(67)$ & $8520(2)$ & $4070(1)$ & $5823(1)$ & $56(1)$ \\
$\mathrm{C}(68)$ & $7942(3)$ & $5046(1)$ & $3949(1)$ & $71(1)$ \\
$\mathrm{C}(69)$ & $8900(3)$ & $5193(2)$ & $4276(2)$ & $109(2)$ \\
$\mathrm{C}(70)$ & $7393(4)$ & $5548(1)$ & $3707(2)$ & $110(2)$ \\
$\mathrm{C}(71)$ & $8253(3)$ & $4700(1)$ & $3434(1)$ & $83(1)$ \\
$\mathrm{C}(72)$ & $4525(2)$ & $4298(1)$ & $4250(1)$ & $73(1)$ \\
$\mathrm{C}(73)$ & $4269(3)$ & $4838(1)$ & $3940(2)$ & $128(2)$ \\
$\mathrm{C}(74)$ & $3887(2)$ & $4280(2)$ & $4770(2)$ & $103(2)$ \\
$\mathrm{C}(75)$ & $4208(2)$ & $3832(1)$ & $3832(1)$ & $65(1)$ \\
\hline
\end{tabular}


Table S3. Bond lengths $[\AA]$ and angles $\left[{ }^{\circ}\right]$ for cain01_sq.

\begin{tabular}{|c|c|}
\hline $\mathrm{P}(1)-\mathrm{C}(3)$ & $1.838(2)$ \\
\hline $\mathrm{P}(1)-\mathrm{C}(28)$ & $1.840(2)$ \\
\hline $\mathrm{P}(1)-\mathrm{C}(53)$ & $1.838(2)$ \\
\hline $\mathrm{P}(2)-\mathrm{C}(1)$ & $1.679(2)$ \\
\hline $\mathrm{P}(2)-\mathrm{C}(8)$ & $1.852(2)$ \\
\hline $\mathrm{P}(3)-\mathrm{C}(26)$ & $1.677(2)$ \\
\hline $\mathrm{P}(3)-\mathrm{C}(33)$ & $1.850(2)$ \\
\hline$P(4)-C(51)$ & $1.677(2)$ \\
\hline $\mathrm{P}(4)-\mathrm{C}(58)$ & $1.860(2)$ \\
\hline $\mathrm{C}(1)-\mathrm{C}(2)$ & $1.467(3)$ \\
\hline $\mathrm{C}(2)-\mathrm{C}(3)$ & $1.412(3)$ \\
\hline$C(2)-C(7)$ & $1.403(3)$ \\
\hline$C(3)-C(4)$ & $1.402(3)$ \\
\hline$C(4)-C(5)$ & $1.377(3)$ \\
\hline$C(5)-C(6)$ & $1.381(3)$ \\
\hline$C(6)-C(7)$ & $1.380(3)$ \\
\hline $\mathrm{C}(8)-\mathrm{C}(9)$ & $1.423(3)$ \\
\hline $\mathrm{C}(8)-\mathrm{C}(13)$ & $1.423(3)$ \\
\hline $\mathrm{C}(9)-\mathrm{C}(10)$ & $1.394(3)$ \\
\hline $\mathrm{C}(9)-\mathrm{C}(14)$ & $1.555(3)$ \\
\hline$C(10)-C(11)$ & $1.383(3)$ \\
\hline $\mathrm{C}(11)-\mathrm{C}(12)$ & $1.391(3)$ \\
\hline $\mathrm{C}(11)-\mathrm{C}(18)$ & $1.537(3)$ \\
\hline $\mathrm{C}(12)-\mathrm{C}(13)$ & $1.389(3)$ \\
\hline $\mathrm{C}(13)-\mathrm{C}(22)$ & $1.563(3)$ \\
\hline$C(14)-C(15)$ & $1.532(4)$ \\
\hline$C(14)-C(16)$ & $1.542(3)$ \\
\hline $\mathrm{C}(14)-\mathrm{C}(17)$ & $1.549(4)$ \\
\hline$C(18)-C(19)$ & $1.535(3)$ \\
\hline $\mathrm{C}(18)-\mathrm{C}(20)$ & $1.537(4)$ \\
\hline$C(18)-C(21)$ & $1.533(4)$ \\
\hline $\mathrm{C}(22)-\mathrm{C}(23)$ & $1.543(3)$ \\
\hline $\mathrm{C}(22)-\mathrm{C}(24)$ & $1.531(3)$ \\
\hline $\mathrm{C}(22)-\mathrm{C}(25)$ & $1.538(3)$ \\
\hline
\end{tabular}




\begin{tabular}{|c|c|}
\hline$C(26)-C(27)$ & $1.463(3)$ \\
\hline$C(27)-C(28)$ & $1.415(3)$ \\
\hline $\mathrm{C}(27)-\mathrm{C}(32)$ & $1.403(3)$ \\
\hline C(28)-C(29) & $1.392(3)$ \\
\hline$C(29)-C(30)$ & $1.384(3)$ \\
\hline $\mathrm{C}(30)-\mathrm{C}(31)$ & $1.382(4)$ \\
\hline $\mathrm{C}(31)-\mathrm{C}(32)$ & $1.375(4)$ \\
\hline$C(33)-C(34)$ & $1.425(3)$ \\
\hline C(33)-C(38) & $1.419(3)$ \\
\hline$C(34)-C(35)$ & $1.390(3)$ \\
\hline C(34)-C(39) & $1.554(3)$ \\
\hline$C(35)-C(36)$ & $1.380(3)$ \\
\hline$C(36)-C(37)$ & $1.387(3)$ \\
\hline C(36)-C(43) & $1.534(3)$ \\
\hline $\mathrm{C}(37)-\mathrm{C}(38)$ & $1.399(3)$ \\
\hline C(38)-C(47) & $1.555(3)$ \\
\hline$C(39)-C(40)$ & $1.538(3)$ \\
\hline $\mathrm{C}(39)-\mathrm{C}(41)$ & $1.536(3)$ \\
\hline$C(39)-C(42)$ & $1.526(3)$ \\
\hline$C(43)-C(44)$ & $1.599(7)$ \\
\hline $\mathrm{C}(43)-\mathrm{C}\left(44^{\prime}\right)$ & $1.460(8)$ \\
\hline C(43)-C(45) & $1.550(6)$ \\
\hline $\mathrm{C}(43)-\mathrm{C}\left(45^{\prime}\right)$ & $1.574(6)$ \\
\hline$C(43)-C(46)$ & $1.495(7)$ \\
\hline$C(43)-C\left(46^{\prime}\right)$ & $1.527(8)$ \\
\hline $\mathrm{C}(47)-\mathrm{C}(48)$ & $1.537(3)$ \\
\hline C(47)-C(49) & $1.539(3)$ \\
\hline$C(47)-C(50)$ & $1.541(3)$ \\
\hline$C(51)-C(52)$ & $1.467(3)$ \\
\hline $\mathrm{C}(52)-\mathrm{C}(53)$ & $1.418(3)$ \\
\hline$C(52)-C(57)$ & $1.397(3)$ \\
\hline $\mathrm{C}(53)-\mathrm{C}(54)$ & $1.393(3)$ \\
\hline $\mathrm{C}(54)-\mathrm{C}(55)$ & $1.382(3)$ \\
\hline$C(55)-C(56)$ & $1.388(3)$ \\
\hline$C(56)-C(57)$ & $1.377(3)$ \\
\hline C(58)-C(59) & $1.418(3)$ \\
\hline
\end{tabular}




\begin{tabular}{|c|c|}
\hline $\mathrm{C}(58)-\mathrm{C}(63)$ & $1.406(3)$ \\
\hline $\mathrm{C}(59)-\mathrm{C}(60)$ & $1.387(3)$ \\
\hline C(59)-C(64) & $1.553(3)$ \\
\hline $\mathrm{C}(60)-\mathrm{C}(61)$ & $1.378(3)$ \\
\hline $\mathrm{C}(61)-\mathrm{C}(62)$ & $1.391(4)$ \\
\hline $\mathrm{C}(61)-\mathrm{C}(68)$ & $1.539(4)$ \\
\hline $\mathrm{C}(62)-\mathrm{C}(63)$ & $1.391(4)$ \\
\hline $\mathrm{C}(63)-\mathrm{C}(72)$ & $1.563(3)$ \\
\hline $\mathrm{C}(64)-\mathrm{C}(65)$ & $1.534(3)$ \\
\hline $\mathrm{C}(64)-\mathrm{C}(66)$ & $1.540(3)$ \\
\hline $\mathrm{C}(64)-\mathrm{C}(67)$ & $1.537(3)$ \\
\hline $\mathrm{C}(68)-\mathrm{C}(69)$ & $1.514(5)$ \\
\hline $\mathrm{C}(68)-\mathrm{C}(70)$ & $1.524(4)$ \\
\hline $\mathrm{C}(68)-\mathrm{C}(71)$ & $1.553(4)$ \\
\hline$C(72)-C(73)$ & $1.534(5)$ \\
\hline $\mathrm{C}(72)-\mathrm{C}(74)$ & $1.539(5)$ \\
\hline C(72)-C(75) & $1.543(3)$ \\
\hline $\mathrm{C}(3)-\mathrm{P}(1)-\mathrm{C}(28)$ & $102.05(9)$ \\
\hline $\mathrm{C}(3)-\mathrm{P}(1)-\mathrm{C}(53)$ & 101.74(9) \\
\hline $\mathrm{C}(53)-\mathrm{P}(1)-\mathrm{C}(28)$ & $100.54(9)$ \\
\hline $\mathrm{C}(1)-\mathrm{P}(2)-\mathrm{C}(8)$ & $100.91(10)$ \\
\hline$C(26)-P(3)-C(33)$ & $100.11(10)$ \\
\hline $\mathrm{C}(51)-\mathrm{P}(4)-\mathrm{C}(58)$ & $99.93(10)$ \\
\hline $\mathrm{C}(2)-\mathrm{C}(1)-\mathrm{P}(2)$ & $123.84(16)$ \\
\hline $\mathrm{C}(3)-\mathrm{C}(2)-\mathrm{C}(1)$ & $121.68(18)$ \\
\hline $\mathrm{C}(7)-\mathrm{C}(2)-\mathrm{C}(1)$ & $120.07(19)$ \\
\hline $\mathrm{C}(7)-\mathrm{C}(2)-\mathrm{C}(3)$ & $118.24(19)$ \\
\hline $\mathrm{C}(2)-\mathrm{C}(3)-\mathrm{P}(1)$ & $119.17(15)$ \\
\hline $\mathrm{C}(4)-\mathrm{C}(3)-\mathrm{P}(1)$ & $121.81(16)$ \\
\hline $\mathrm{C}(4)-\mathrm{C}(3)-\mathrm{C}(2)$ & 118.99(19) \\
\hline $\mathrm{C}(5)-\mathrm{C}(4)-\mathrm{C}(3)$ & 121.1(2) \\
\hline$C(4)-C(5)-C(6)$ & $120.4(2)$ \\
\hline$C(7)-C(6)-C(5)$ & $119.5(2)$ \\
\hline $\mathrm{C}(6)-\mathrm{C}(7)-\mathrm{C}(2)$ & $121.8(2)$ \\
\hline $\mathrm{C}(9)-\mathrm{C}(8)-\mathrm{P}(2)$ & $120.46(17)$ \\
\hline
\end{tabular}




$\begin{array}{ll}\mathrm{C}(9)-\mathrm{C}(8)-\mathrm{C}(13) & 119.5(2) \\ \mathrm{C}(13)-\mathrm{C}(8)-\mathrm{P}(2) & 119.87(16) \\ \mathrm{C}(8)-\mathrm{C}(9)-\mathrm{C}(14) & 124.2(2) \\ \mathrm{C}(10)-\mathrm{C}(9)-\mathrm{C}(8) & 117.8(2) \\ \mathrm{C}(10)-\mathrm{C}(9)-\mathrm{C}(14) & 118.0(2) \\ \mathrm{C}(11)-\mathrm{C}(10)-\mathrm{C}(9) & 123.5(2) \\ \mathrm{C}(10)-\mathrm{C}(11)-\mathrm{C}(12) & 117.1(2) \\ \mathrm{C}(10)-\mathrm{C}(11)-\mathrm{C}(18) & 122.8(2) \\ \mathrm{C}(12)-\mathrm{C}(11)-\mathrm{C}(18) & 120.1(2) \\ \mathrm{C}(13)-\mathrm{C}(12)-\mathrm{C}(11) & 123.0(2) \\ \mathrm{C}(8)-\mathrm{C}(13)-\mathrm{C}(22) & 124.40(19) \\ \mathrm{C}(12)-\mathrm{C}(13)-\mathrm{C}(8) & 118.2(2) \\ \mathrm{C}(12)-\mathrm{C}(13)-\mathrm{C}(22) & 117.3(2) \\ \mathrm{C}(15)-\mathrm{C}(14)-\mathrm{C}(9) & 111.2(2) \\ \mathrm{C}(15)-\mathrm{C}(14)-\mathrm{C}(16) & 110.7(2) \\ \mathrm{C}(15)-\mathrm{C}(14)-\mathrm{C}(17) & 106.0(2) \\ \mathrm{C}(16)-\mathrm{C}(14)-\mathrm{C}(9) & 111.43(19) \\ \mathrm{C}(16)-\mathrm{C}(14)-\mathrm{C}(17) & 105.7(2) \\ \mathrm{C}(17)-\mathrm{C}(14)-\mathrm{C}(9) & 111.6(2) \\ \mathrm{C}(11)-\mathrm{C}(18)-\mathrm{C}(20) & 111.23(19) \\ \mathrm{C}(19)-\mathrm{C}(18)-\mathrm{C}(11) & 107.69(19) \\ \mathrm{C}(19)-\mathrm{C}(18)-\mathrm{C}(20) & 108.3(2) \\ \mathrm{C}(21)-\mathrm{C}(18)-\mathrm{C}(11) & 112.2(2) \\ \mathrm{C}(21)-\mathrm{C}(18)-\mathrm{C}(19) & 109.1(2) \\ \mathrm{C}(21)-\mathrm{C}(18)-\mathrm{C}(20) & 108.3(2) \\ \mathrm{C}(23)-\mathrm{C}(22)-\mathrm{C}(13) & 110.20(18) \\ \mathrm{C}(24)-\mathrm{C}(22)-\mathrm{C}(13) & 111.54(19) \\ \mathrm{C}(24)-\mathrm{C}(22)-\mathrm{C}(23) & 105.7(2) \\ \mathrm{C}(24)-\mathrm{C}(22)-\mathrm{C}(25) & 106.82(19) \\ \mathrm{C}(25)-\mathrm{C}(22)-\mathrm{C}(13) & 112.57(19) \\ \mathrm{C}(25)-\mathrm{C}(22)-\mathrm{C}(23) & 109.78(19) \\ \mathrm{C}(27)-\mathrm{C}(26)-\mathrm{P}(3) & 124.06(16) \\ \mathrm{C}(28)-\mathrm{C}(27)-\mathrm{C}(26) & 121.32(18) \\ \mathrm{C}(32)-\mathrm{C}(27)-\mathrm{C}(28) & \\ \mathrm{C}(27)-\mathrm{C}(28)-\mathrm{P}(1) & \\ & \end{array}$




\begin{tabular}{|c|c|}
\hline C(29)-C(28)-P(1) & $122.07(16)$ \\
\hline $\mathrm{C}(29)-\mathrm{C}(28)-\mathrm{C}(27)$ & $119.46(19)$ \\
\hline $\mathrm{C}(30)-\mathrm{C}(29)-\mathrm{C}(28)$ & $121.2(2)$ \\
\hline $\mathrm{C}(31)-\mathrm{C}(30)-\mathrm{C}(29)$ & $119.6(2)$ \\
\hline $\mathrm{C}(32)-\mathrm{C}(31)-\mathrm{C}(30)$ & $120.3(2)$ \\
\hline$C(31)-C(32)-C(27)$ & $121.5(2)$ \\
\hline $\mathrm{C}(34)-\mathrm{C}(33)-\mathrm{P}(3)$ & $119.80(16)$ \\
\hline $\mathrm{C}(38)-\mathrm{C}(33)-\mathrm{P}(3)$ & $120.52(16)$ \\
\hline $\mathrm{C}(38)-\mathrm{C}(33)-\mathrm{C}(34)$ & $119.4(2)$ \\
\hline C(33)-C(34)-C(39) & $123.83(19)$ \\
\hline$C(35)-C(34)-C(33)$ & $118.04(19)$ \\
\hline$C(35)-C(34)-C(39)$ & $118.03(19)$ \\
\hline$C(36)-C(35)-C(34)$ & $123.5(2)$ \\
\hline$C(35)-C(36)-C(37)$ & $117.2(2)$ \\
\hline$C(35)-C(36)-C(43)$ & $121.1(2)$ \\
\hline$C(37)-C(36)-C(43)$ & $121.6(2)$ \\
\hline $\mathrm{C}(36)-\mathrm{C}(37)-\mathrm{C}(38)$ & $123.0(2)$ \\
\hline $\mathrm{C}(33)-\mathrm{C}(38)-\mathrm{C}(47)$ & $123.88(19)$ \\
\hline$C(37)-C(38)-C(33)$ & $118.0(2)$ \\
\hline $\mathrm{C}(37)-\mathrm{C}(38)-\mathrm{C}(47)$ & $118.06(19)$ \\
\hline $\mathrm{C}(40)-\mathrm{C}(39)-\mathrm{C}(34)$ & $109.64(17)$ \\
\hline C(41)-C(39)-C(34) & $112.55(18)$ \\
\hline$C(41)-C(39)-C(40)$ & $110.4(2)$ \\
\hline $\mathrm{C}(42)-\mathrm{C}(39)-\mathrm{C}(34)$ & $111.7(2)$ \\
\hline $\mathrm{C}(42)-\mathrm{C}(39)-\mathrm{C}(40)$ & $105.6(2)$ \\
\hline $\mathrm{C}(42)-\mathrm{C}(39)-\mathrm{C}(41)$ & $106.69(18)$ \\
\hline$C(36)-C(43)-C(44)$ & 107.6(3) \\
\hline$C(36)-C(43)-C(45)$ & $112.5(3)$ \\
\hline $\mathrm{C}(36)-\mathrm{C}(43)-\mathrm{C}\left(45^{\prime}\right)$ & $103.7(3)$ \\
\hline $\mathrm{C}\left(44^{\prime}\right)-\mathrm{C}(43)-\mathrm{C}(36)$ & $112.0(3)$ \\
\hline $\mathrm{C}\left(44^{\prime}\right)-\mathrm{C}(43)-\mathrm{C}\left(45^{\prime}\right)$ & $111.3(4)$ \\
\hline $\mathrm{C}\left(44^{\prime}\right)-\mathrm{C}(43)-\mathrm{C}\left(46^{\prime}\right)$ & $112.1(5)$ \\
\hline $\mathrm{C}(45)-\mathrm{C}(43)-\mathrm{C}(44)$ & 104.1(4) \\
\hline$C(46)-C(43)-C(36)$ & $115.5(3)$ \\
\hline $\mathrm{C}(46)-\mathrm{C}(43)-\mathrm{C}(44)$ & $106.4(5)$ \\
\hline $\mathrm{C}(46)-\mathrm{C}(43)-\mathrm{C}(45)$ & $110.0(5)$ \\
\hline
\end{tabular}




$\begin{array}{ll}\mathrm{C}(46)-\mathrm{C}(43)-\mathrm{C}(36) & 109.3(3) \\ \mathrm{C}(46)-\mathrm{C}(43)-\mathrm{C}(45) & 107.9(5) \\ \mathrm{C}(48)-\mathrm{C}(47)-\mathrm{C}(38) & 111.33(19) \\ \mathrm{C}(48)-\mathrm{C}(47)-\mathrm{C}(49) & 106.49(18) \\ \mathrm{C}(48)-\mathrm{C}(47)-\mathrm{C}(50) & 105.37(19) \\ \mathrm{C}(49)-\mathrm{C}(47)-\mathrm{C}(38) & 112.51(18) \\ \mathrm{C}(49)-\mathrm{C}(47)-\mathrm{C}(50) & 110.1(2) \\ \mathrm{C}(50)-\mathrm{C}(47)-\mathrm{C}(38) & 110.68(17) \\ \mathrm{C}(52)-\mathrm{C}(51)-\mathrm{P}(4) & 124.12(16) \\ \mathrm{C}(53)-\mathrm{C}(52)-\mathrm{C}(51) & 121.03(18) \\ \mathrm{C}(57)-\mathrm{C}(52)-\mathrm{C}(51) & 120.63(19) \\ \mathrm{C}(57)-\mathrm{C}(52)-\mathrm{C}(53) & 118.31(19) \\ \mathrm{C}(52)-\mathrm{C}(53)-\mathrm{P}(1) & 118.09(15) \\ \mathrm{C}(54)-\mathrm{C}(53)-\mathrm{P}(1) & 122.90(16) \\ \mathrm{C}(54)-\mathrm{C}(53)-\mathrm{C}(52) & 119.00(19) \\ \mathrm{C}(55)-\mathrm{C}(54)-\mathrm{C}(53) & 121.4(2) \\ \mathrm{C}(54)-\mathrm{C}(55)-\mathrm{C}(56) & 119.8(2) \\ \mathrm{C}(57)-\mathrm{C}(56)-\mathrm{C}(55) & 119.7(2) \\ \mathrm{C}(56)-\mathrm{C}(57)-\mathrm{C}(52) & 121.8(2) \\ \mathrm{C}(59)-\mathrm{C}(58)-\mathrm{P}(4) & 120.39(16) \\ \mathrm{C}(63)-\mathrm{C}(58)-\mathrm{P}(4) & 119.82(17) \\ \mathrm{C}(63)-\mathrm{C}(58)-\mathrm{C}(59) & 119.6(2) \\ \mathrm{C}(58)-\mathrm{C}(59)-\mathrm{C}(64) & 123.82(19) \\ \mathrm{C}(60)-\mathrm{C}(59)-\mathrm{C}(58) & 118.56(19) \\ \mathrm{C}(60)-\mathrm{C}(59)-\mathrm{C}(64) & 117.62(19) \\ \mathrm{C}(61)-\mathrm{C}(60)-\mathrm{C}(59) & 123.0(2) \\ \mathrm{C}(60)-\mathrm{C}(61)-\mathrm{C}(62) & 116.8(2) \\ \mathrm{C}(60)-\mathrm{C}(61)-\mathrm{C}(68) & 121.6(2) \\ \mathrm{C}(62)-\mathrm{C}(61)-\mathrm{C}(68) & 121.6(2) \\ \mathrm{C}(63)-\mathrm{C}(62)-\mathrm{C}(61) & 123.5(2) \\ \mathrm{C}(58)-\mathrm{C}(63)-\mathrm{C}(72) & 124.5(2) \\ \mathrm{C}(62)-\mathrm{C}(63)-\mathrm{C}(58) & 117.8(2) \\ \mathrm{C}(62)-\mathrm{C}(63)-\mathrm{C}(72) & 117.7(2) \\ & \end{array}$




\begin{tabular}{ll}
$\mathrm{C}(66)-\mathrm{C}(64)-\mathrm{C}(59)$ & $110.53(18)$ \\
$\mathrm{C}(67)-\mathrm{C}(64)-\mathrm{C}(59)$ & $111.49(18)$ \\
$\mathrm{C}(67)-\mathrm{C}(64)-\mathrm{C}(66)$ & $106.87(19)$ \\
$\mathrm{C}(61)-\mathrm{C}(68)-\mathrm{C}(71)$ & $108.2(2)$ \\
$\mathrm{C}(69)-\mathrm{C}(68)-\mathrm{C}(61)$ & $112.5(2)$ \\
$\mathrm{C}(69)-\mathrm{C}(68)-\mathrm{C}(70)$ & $112.6(3)$ \\
$\mathrm{C}(69)-\mathrm{C}(68)-\mathrm{C}(71)$ & $104.9(3)$ \\
$\mathrm{C}(70)-\mathrm{C}(68)-\mathrm{C}(61)$ & $111.1(3)$ \\
$\mathrm{C}(70)-\mathrm{C}(68)-\mathrm{C}(71)$ & $107.1(3)$ \\
$\mathrm{C}(73)-\mathrm{C}(72)-\mathrm{C}(63)$ & $110.1(3)$ \\
$\mathrm{C}(73)-\mathrm{C}(72)-\mathrm{C}(74)$ & $106.1(3)$ \\
$\mathrm{C}(73)-\mathrm{C}(72)-\mathrm{C}(75)$ & $106.4(2)$ \\
$\mathrm{C}(74)-\mathrm{C}(72)-\mathrm{C}(63)$ & $112.0(2)$ \\
$\mathrm{C}(74)-\mathrm{C}(72)-\mathrm{C}(75)$ & $109.6(3)$ \\
$\mathrm{C}(75)-\mathrm{C}(72)-\mathrm{C}(63)$ & $112.3(2)$ \\
\hline
\end{tabular}

Symmetry transformations used to generate equivalent atoms: 
Table S4. Anisotropic displacement parameters $\left(\AA^{2} \times 10^{3}\right)$ for cain01_sq. The anisotropic displacement factor exponent takes the form: $-2 \pi^{2}\left[h^{2} a^{* 2} U^{11}+\ldots+2 h k a^{*} b^{*} U^{12}\right]$

\begin{tabular}{|c|c|c|c|c|c|c|}
\hline & $\mathrm{U}^{11}$ & $\mathrm{U}^{22}$ & $\mathrm{U}^{33}$ & $U^{23}$ & $\mathrm{U}^{13}$ & $\mathrm{U}^{12}$ \\
\hline $\mathrm{P}(1)$ & $34(1)$ & $38(1)$ & $34(1)$ & $-2(1)$ & $3(1)$ & $2(1)$ \\
\hline $\mathrm{P}(2)$ & $36(1)$ & $56(1)$ & $48(1)$ & $10(1)$ & $7(1)$ & $5(1)$ \\
\hline $\mathrm{P}(3)$ & $47(1)$ & $45(1)$ & $38(1)$ & $-4(1)$ & $-1(1)$ & $-8(1)$ \\
\hline $\mathrm{P}(4)$ & $52(1)$ & $52(1)$ & $62(1)$ & $-19(1)$ & $21(1)$ & $-9(1)$ \\
\hline $\mathrm{C}(1)$ & $39(1)$ & $42(1)$ & $39(1)$ & 1(1) & $2(1)$ & $2(1)$ \\
\hline $\mathrm{C}(2)$ & $38(1)$ & $40(1)$ & $37(1)$ & $-1(1)$ & $4(1)$ & $2(1)$ \\
\hline$C(3)$ & $38(1)$ & $40(1)$ & $36(1)$ & $-1(1)$ & $2(1)$ & $2(1)$ \\
\hline $\mathrm{C}(4)$ & $40(1)$ & $55(1)$ & $40(1)$ & $3(1)$ & $6(1)$ & $-1(1)$ \\
\hline$C(5)$ & $50(1)$ & $65(2)$ & $40(1)$ & $8(1)$ & $6(1)$ & $2(1)$ \\
\hline$C(6)$ & $50(1)$ & $62(1)$ & $40(1)$ & $5(1)$ & $-3(1)$ & $5(1)$ \\
\hline$C(7)$ & $37(1)$ & $52(1)$ & $45(1)$ & $3(1)$ & $-1(1)$ & $2(1)$ \\
\hline $\mathrm{C}(8)$ & $30(1)$ & $52(1)$ & $44(1)$ & $4(1)$ & $8(1)$ & $4(1)$ \\
\hline $\mathrm{C}(9)$ & $31(1)$ & $51(1)$ & $48(1)$ & $2(1)$ & $7(1)$ & $-2(1)$ \\
\hline $\mathrm{C}(10)$ & $32(1)$ & $57(1)$ & $45(1)$ & $-2(1)$ & $4(1)$ & $-3(1)$ \\
\hline $\mathrm{C}(11)$ & $30(1)$ & $56(1)$ & $43(1)$ & $7(1)$ & $4(1)$ & $1(1)$ \\
\hline $\mathrm{C}(12)$ & $35(1)$ & $47(1)$ & $50(1)$ & $3(1)$ & $6(1)$ & $3(1)$ \\
\hline$C(13)$ & $31(1)$ & $53(1)$ & $44(1)$ & $2(1)$ & $8(1)$ & $4(1)$ \\
\hline$C(14)$ & $47(1)$ & $49(1)$ & $56(1)$ & $-1(1)$ & 11(1) & $-4(1)$ \\
\hline$C(15)$ & $65(2)$ & $51(1)$ & $77(2)$ & $3(1)$ & $9(1)$ & $10(1)$ \\
\hline$C(16)$ & $59(2)$ & $61(2)$ & $74(2)$ & $-10(1)$ & $23(1)$ & $-16(1)$ \\
\hline $\mathrm{C}(17)$ & $60(2)$ & $54(1)$ & $67(2)$ & $-9(1)$ & $19(1)$ & $-7(1)$ \\
\hline$C(18)$ & $43(1)$ & $61(1)$ & $46(1)$ & $10(1)$ & $-2(1)$ & $-3(1)$ \\
\hline$C(19)$ & $56(2)$ & $60(2)$ & $60(2)$ & $6(1)$ & $-7(1)$ & $8(1)$ \\
\hline$C(20)$ & $66(2)$ & $82(2)$ & $56(2)$ & $25(1)$ & $-7(1)$ & $-20(1)$ \\
\hline $\mathrm{C}(21)$ & $61(2)$ & $78(2)$ & $46(1)$ & 11(1) & $3(1)$ & $4(1)$ \\
\hline $\mathrm{C}(22)$ & $43(1)$ & $52(1)$ & $42(1)$ & $-2(1)$ & $7(1)$ & $3(1)$ \\
\hline $\mathrm{C}(23)$ & $47(1)$ & $66(2)$ & $48(1)$ & $-6(1)$ & $-1(1)$ & $4(1)$ \\
\hline $\mathrm{C}(24)$ & $47(1)$ & $59(1)$ & $54(1)$ & $-5(1)$ & $7(1)$ & $-4(1)$ \\
\hline$C(25)$ & $52(1)$ & $53(1)$ & $50(1)$ & $-4(1)$ & $14(1)$ & $2(1)$ \\
\hline$C(26)$ & $40(1)$ & $42(1)$ & $36(1)$ & $-6(1)$ & $9(1)$ & $-3(1)$ \\
\hline $\mathrm{C}(27)$ & $39(1)$ & $42(1)$ & $42(1)$ & $-5(1)$ & $9(1)$ & $-1(1)$ \\
\hline
\end{tabular}




\begin{tabular}{|c|c|c|c|c|c|c|}
\hline$C(28)$ & $38(1)$ & $41(1)$ & $40(1)$ & $-5(1)$ & $8(1)$ & 1(1) \\
\hline$C(29)$ & $57(1)$ & $44(1)$ & $45(1)$ & $1(1)$ & $6(1)$ & $7(1)$ \\
\hline$C(30)$ & $79(2)$ & $42(1)$ & $57(1)$ & $2(1)$ & $6(1)$ & $12(1)$ \\
\hline $\mathrm{C}(31)$ & $76(2)$ & $41(1)$ & $61(2)$ & $-10(1)$ & $8(1)$ & $5(1)$ \\
\hline$C(32)$ & $56(1)$ & $47(1)$ & $45(1)$ & $-8(1)$ & $6(1)$ & $1(1)$ \\
\hline$C(33)$ & $36(1)$ & $47(1)$ & $34(1)$ & $-3(1)$ & $-5(1)$ & $-5(1)$ \\
\hline$C(34)$ & $38(1)$ & $50(1)$ & $32(1)$ & $-5(1)$ & $-3(1)$ & $-5(1)$ \\
\hline$C(35)$ & $41(1)$ & $51(1)$ & $39(1)$ & $-5(1)$ & $-3(1)$ & $1(1)$ \\
\hline$C(36)$ & $44(1)$ & $47(1)$ & $44(1)$ & $1(1)$ & $-7(1)$ & $-3(1)$ \\
\hline$C(37)$ & $37(1)$ & $55(1)$ & $42(1)$ & $5(1)$ & $-1(1)$ & $-8(1)$ \\
\hline $\mathrm{C}(38)$ & $34(1)$ & $51(1)$ & $36(1)$ & $-1(1)$ & $-4(1)$ & $-4(1)$ \\
\hline C(39) & $39(1)$ & $60(1)$ & $36(1)$ & $-9(1)$ & $5(1)$ & $-8(1)$ \\
\hline $\mathrm{C}(40)$ & $45(1)$ & $80(2)$ & $52(1)$ & $-17(1)$ & $10(1)$ & $-21(1)$ \\
\hline$C(41)$ & $52(1)$ & $64(2)$ & $41(1)$ & $-4(1)$ & $12(1)$ & $-5(1)$ \\
\hline$C(42)$ & $39(1)$ & $77(2)$ & $49(1)$ & $-10(1)$ & $3(1)$ & $-1(1)$ \\
\hline$C(43)$ & $65(2)$ & $49(1)$ & $58(2)$ & $1(1)$ & $0(1)$ & $-6(1)$ \\
\hline C(44) & 101(5) & $49(3)$ & $68(4)$ & $0(3)$ & $-20(4)$ & $12(3)$ \\
\hline$C\left(44^{\prime}\right)$ & $97(5)$ & $49(3)$ & $140(7)$ & $10(4)$ & $-23(5)$ & $9(3)$ \\
\hline$C(45)$ & $86(4)$ & $46(3)$ & $52(3)$ & $-8(2)$ & $4(3)$ & $-9(3)$ \\
\hline$C\left(45^{\prime}\right)$ & $84(4)$ & $48(3)$ & $82(4)$ & $-13(3)$ & $-1(3)$ & $-13(3)$ \\
\hline$C(46)$ & $87(5)$ & $40(3)$ & $132(8)$ & $-5(4)$ & $45(5)$ & $-10(3)$ \\
\hline$C\left(46^{\prime}\right)$ & $170(9)$ & $63(4)$ & $85(5)$ & $20(3)$ & $-11(5)$ & $-44(5)$ \\
\hline$C(47)$ & $35(1)$ & $57(1)$ & $43(1)$ & $-4(1)$ & $3(1)$ & $-4(1)$ \\
\hline $\mathrm{C}(48)$ & $40(1)$ & $69(2)$ & $44(1)$ & $3(1)$ & $5(1)$ & $-2(1)$ \\
\hline$C(49)$ & $38(1)$ & $57(1)$ & $49(1)$ & $0(1)$ & $5(1)$ & $1(1)$ \\
\hline $\mathrm{C}(50)$ & $46(1)$ & $66(2)$ & $47(1)$ & $-13(1)$ & $9(1)$ & $-5(1)$ \\
\hline $\mathrm{C}(51)$ & $36(1)$ & $41(1)$ & $42(1)$ & $-2(1)$ & $3(1)$ & $4(1)$ \\
\hline$C(52)$ & $38(1)$ & $42(1)$ & $37(1)$ & $-1(1)$ & $3(1)$ & $2(1)$ \\
\hline$C(53)$ & $34(1)$ & $45(1)$ & $35(1)$ & $-2(1)$ & $2(1)$ & 1(1) \\
\hline$C(54)$ & $45(1)$ & $43(1)$ & $44(1)$ & $-6(1)$ & $4(1)$ & $-2(1)$ \\
\hline$C(55)$ & $47(1)$ & $53(1)$ & $51(1)$ & $-6(1)$ & $7(1)$ & $-11(1)$ \\
\hline$C(56)$ & $38(1)$ & $60(1)$ & $51(1)$ & $-4(1)$ & $9(1)$ & $-5(1)$ \\
\hline$C(57)$ & $41(1)$ & $50(1)$ & $44(1)$ & $-5(1)$ & $6(1)$ & 2(1) \\
\hline$C(58)$ & $42(1)$ & $38(1)$ & $48(1)$ & $-9(1)$ & $7(1)$ & $4(1)$ \\
\hline$C(59)$ & $42(1)$ & $38(1)$ & $40(1)$ & $-2(1)$ & $6(1)$ & $6(1)$ \\
\hline $\mathrm{C}(60)$ & $43(1)$ & $43(1)$ & $38(1)$ & $0(1)$ & $3(1)$ & $3(1)$ \\
\hline
\end{tabular}




\begin{tabular}{llllcll}
$\mathrm{C}(61)$ & $69(2)$ & $42(1)$ & $39(1)$ & $1(1)$ & $0(1)$ & $-1(1)$ \\
$\mathrm{C}(62)$ & $80(2)$ & $45(1)$ & $41(1)$ & $-5(1)$ & $-21(1)$ & $12(1)$ \\
$\mathrm{C}(63)$ & $52(1)$ & $40(1)$ & $58(1)$ & $-17(1)$ & $-12(1)$ & $8(1)$ \\
$\mathrm{C}(64)$ & $48(1)$ & $45(1)$ & $41(1)$ & $8(1)$ & $6(1)$ & $10(1)$ \\
$\mathrm{C}(65)$ & $65(2)$ & $66(2)$ & $45(1)$ & $13(1)$ & $10(1)$ & $24(1)$ \\
$\mathrm{C}(66)$ & $52(1)$ & $49(1)$ & $55(1)$ & $9(1)$ & $10(1)$ & $14(1)$ \\
$\mathrm{C}(67)$ & $60(2)$ & $60(2)$ & $46(1)$ & $6(1)$ & $-9(1)$ & $9(1)$ \\
$\mathrm{C}(68)$ & $101(2)$ & $60(2)$ & $52(2)$ & $16(1)$ & $5(2)$ & $-9(2)$ \\
$\mathrm{C}(69)$ & $135(4)$ & $104(3)$ & $90(2)$ & $13(2)$ & $18(2)$ & $-64(3)$ \\
$\mathrm{C}(70)$ & $164(4)$ & $58(2)$ & $117(3)$ & $41(2)$ & $71(3)$ & $34(2)$ \\
$\mathrm{C}(71)$ & $104(3)$ & $79(2)$ & $70(2)$ & $22(2)$ & $31(2)$ & $16(2)$ \\
$\mathrm{C}(72)$ & $58(2)$ & $55(2)$ & $101(2)$ & $-34(2)$ & $-35(2)$ & $16(1)$ \\
$\mathrm{C}(73)$ & $104(3)$ & $51(2)$ & $214(5)$ & $-36(2)$ & $-102(3)$ & $23(2)$ \\
$\mathrm{C}(74)$ & $42(2)$ & $126(3)$ & $138(3)$ & $-86(3)$ & $-18(2)$ & $22(2)$ \\
$\mathrm{C}(75)$ & $67(2)$ & $50(1)$ & $74(2)$ & $-17(1)$ & $-31(1)$ & $8(1)$ \\
\hline
\end{tabular}


Table S5. Hydrogen coordinates ( $\left.\mathrm{x} 10^{4}\right)$ and isotropic displacement parameters $\left(\AA^{2} \mathrm{x} 10^{3}\right)$ for cain01_sq.

\begin{tabular}{|c|c|c|c|c|}
\hline & $\mathrm{x}$ & $\mathrm{y}$ & $\mathrm{z}$ & $\mathrm{U}(\mathrm{eq})$ \\
\hline $\mathrm{H}(1)$ & 8417 & 2340 & 3918 & 48 \\
\hline $\mathrm{H}(4)$ & 6238 & 1630 & 5221 & 54 \\
\hline $\mathrm{H}(5)$ & 7223 & 1501 & 6051 & 61 \\
\hline $\mathrm{H}(6)$ & 8913 & 1672 & 6065 & 60 \\
\hline $\mathrm{H}(7)$ & 9616 & 1957 & 5239 & 54 \\
\hline $\mathrm{H}(10)$ & 9752 & 2333 & 2047 & 53 \\
\hline $\mathrm{H}(12)$ & 10154 & 3728 & 2937 & 53 \\
\hline $\mathrm{H}(15 \mathrm{~A})$ & 10859 & 903 & 3085 & 96 \\
\hline $\mathrm{H}(15 \mathrm{~B})$ & 10797 & 1361 & 3570 & 96 \\
\hline $\mathrm{H}(15 \mathrm{C})$ & 11463 & 1464 & 3041 & 96 \\
\hline $\mathrm{H}(16 \mathrm{~A})$ & 8971 & 919 & 2910 & 96 \\
\hline $\mathrm{H}(16 \mathrm{~B})$ & 8435 & 1499 & 2807 & 96 \\
\hline $\mathrm{H}(16 \mathrm{C})$ & 8950 & 1354 & 3421 & 96 \\
\hline $\mathrm{H}(17 \mathrm{~A})$ & 10122 & 989 & 2174 & 90 \\
\hline $\mathrm{H}(17 \mathrm{~B})$ & 10628 & 1566 & 2046 & 90 \\
\hline $\mathrm{H}(17 \mathrm{C})$ & 9455 & 1503 & 1971 & 90 \\
\hline $\mathrm{H}(19 \mathrm{~A})$ & 8149 & 3393 & 1921 & 88 \\
\hline $\mathrm{H}(19 \mathrm{~B})$ & 8486 & 3909 & 1558 & 88 \\
\hline $\mathrm{H}(19 \mathrm{C})$ & 8632 & 3918 & 2240 & 88 \\
\hline $\mathrm{H}(20 \mathrm{~A})$ & 10287 & 4102 & 1474 & 103 \\
\hline $\mathrm{H}(20 \mathrm{~B})$ & 11090 & 3743 & 1835 & 103 \\
\hline $\mathrm{H}(20 \mathrm{C})$ & 10373 & 4145 & 2156 & 103 \\
\hline $\mathrm{H}(21 \mathrm{~A})$ & 9188 & 2796 & 1327 & 92 \\
\hline $\mathrm{H}(21 \mathrm{~B})$ & 10342 & 2918 & 1299 & 92 \\
\hline $\mathrm{H}(21 \mathrm{C})$ & 9549 & 3321 & 987 & 92 \\
\hline $\mathrm{H}(23 \mathrm{~A})$ & 11824 & 3318 & 4590 & 81 \\
\hline $\mathrm{H}(23 \mathrm{~B})$ & 12076 & 3055 & 3991 & 81 \\
\hline $\mathrm{H}(23 \mathrm{C})$ & 11375 & 2733 & 4401 & 81 \\
\hline $\mathrm{H}(24 \mathrm{~A})$ & 11304 & 4141 & 4151 & 80 \\
\hline $\mathrm{H}(24 \mathrm{~B})$ & 10449 & 4172 & 3652 & 80 \\
\hline
\end{tabular}




\begin{tabular}{|c|c|c|c|c|}
\hline $\mathrm{H}(24 \mathrm{C})$ & 11504 & 3923 & 3526 & 80 \\
\hline $\mathrm{H}(25 \mathrm{~A})$ & 10111 & 3721 & 4705 & 77 \\
\hline $\mathrm{H}(25 \mathrm{~B})$ & 9712 & 3112 & 4579 & 77 \\
\hline $\mathrm{H}(25 \mathrm{C})$ & 9267 & 3617 & 4211 & 77 \\
\hline $\mathrm{H}(26)$ & 6135 & 2128 & 2922 & 47 \\
\hline $\mathrm{H}(29)$ & 7292 & 886 & 4342 & 58 \\
\hline $\mathrm{H}(30)$ & 7302 & 43 & 3874 & 71 \\
\hline $\mathrm{H}(31)$ & 6693 & -20 & 2924 & 71 \\
\hline $\mathrm{H}(32)$ & 6104 & 753 & 2443 & 59 \\
\hline $\mathrm{H}(35)$ & 3971 & 3631 & 2401 & 52 \\
\hline $\mathrm{H}(37)$ & 6297 & 3445 & 1455 & 54 \\
\hline $\mathrm{H}(40 \mathrm{~A})$ & 2210 & 2092 & 2312 & 88 \\
\hline $\mathrm{H}(40 \mathrm{~B})$ & 3248 & 1880 & 2112 & 88 \\
\hline $\mathrm{H}(40 \mathrm{C})$ & 2686 & 2371 & 1777 & 88 \\
\hline $\mathrm{H}(41 \mathrm{~A})$ & 2978 & 2281 & 3321 & 78 \\
\hline $\mathrm{H}(41 \mathrm{~B})$ & 3979 & 2627 & 3387 & 78 \\
\hline $\mathrm{H}(41 \mathrm{C})$ & 3978 & 2028 & 3106 & 78 \\
\hline $\mathrm{H}(42 \mathrm{~A})$ & 2005 & 2925 & 2752 & 83 \\
\hline $\mathrm{H}(42 \mathrm{~B})$ & 2499 & 3277 & 2269 & 83 \\
\hline $\mathrm{H}(42 \mathrm{C})$ & 2891 & 3338 & 2923 & 83 \\
\hline $\mathrm{H}(44 \mathrm{~A})$ & 3793 & 4370 & 1631 & 111 \\
\hline $\mathrm{H}(44 \mathrm{~B})$ & 4452 & 4894 & 1499 & 111 \\
\hline $\mathrm{H}(44 \mathrm{C})$ & 4513 & 4352 & 1116 & 111 \\
\hline $\mathrm{H}(44 \mathrm{D})$ & 4203 & 4431 & 2439 & 145 \\
\hline $\mathrm{H}(44 \mathrm{E})$ & 4509 & 4943 & 2061 & 145 \\
\hline $\mathrm{H}(44 \mathrm{~F})$ & 3867 & 4454 & 1774 & 145 \\
\hline $\mathrm{H}(45 \mathrm{~A})$ & 4505 & 4409 & 2648 & 92 \\
\hline $\mathrm{H}(45 \mathrm{~B})$ & 5672 & 4457 & 2781 & 92 \\
\hline $\mathrm{H}(45 \mathrm{C})$ & 5084 & 4942 & 2450 & 92 \\
\hline $\mathrm{H}(45 \mathrm{D})$ & 6760 & 4192 & 2284 & 108 \\
\hline $\mathrm{H}(45 \mathrm{E})$ & 6295 & 4784 & 2394 & 108 \\
\hline $\mathrm{H}(45 \mathrm{~F})$ & 5979 & 4266 & 2761 & 108 \\
\hline $\mathrm{H}(46 \mathrm{~A})$ & 6244 & 4866 & 1703 & 127 \\
\hline $\mathrm{H}(46 \mathrm{~B})$ & 6800 & 4329 & 1949 & 127 \\
\hline $\mathrm{H}(46 \mathrm{C})$ & 6297 & 4328 & 1315 & 127 \\
\hline $\mathrm{H}(46 \mathrm{D})$ & 6246 & 4225 & 1270 & 160 \\
\hline
\end{tabular}




\begin{tabular}{|c|c|c|c|c|}
\hline $\mathrm{H}(46 \mathrm{E})$ & 5125 & 4331 & 1048 & 160 \\
\hline $\mathrm{H}(46 \mathrm{~F})$ & 5766 & 4820 & 1335 & 160 \\
\hline $\mathrm{H}(48 \mathrm{~A})$ & 7197 & 2492 & 600 & 76 \\
\hline $\mathrm{H}(48 \mathrm{~B})$ & 7293 & 2996 & 1037 & 76 \\
\hline $\mathrm{H}(48 \mathrm{C})$ & 6306 & 2918 & 637 & 76 \\
\hline $\mathrm{H}(49 \mathrm{~A})$ & 7728 & 1943 & 1405 & 72 \\
\hline $\mathrm{H}(49 \mathrm{~B})$ & 7038 & 1830 & 1922 & 72 \\
\hline $\mathrm{H}(49 \mathrm{C})$ & 7631 & 2396 & 1892 & 72 \\
\hline $\mathrm{H}(50 \mathrm{~A})$ & 6217 & 1734 & 728 & 79 \\
\hline $\mathrm{H}(50 \mathrm{~B})$ & 5236 & 2091 & 775 & 79 \\
\hline $\mathrm{H}(50 \mathrm{C})$ & 5555 & 1656 & 1263 & 79 \\
\hline $\mathrm{H}(51)$ & 5908 & 3003 & 4341 & 48 \\
\hline $\mathrm{H}(54)$ & 5007 & 1148 & 4073 & 53 \\
\hline $\mathrm{H}(55)$ & 3422 & 1111 & 4391 & 60 \\
\hline $\mathrm{H}(56)$ & 2764 & 1877 & 4834 & 59 \\
\hline $\mathrm{H}(57)$ & 3688 & 2673 & 4947 & 54 \\
\hline $\mathrm{H}(60)$ & 8291 & 4477 & 4919 & 50 \\
\hline $\mathrm{H}(62)$ & 6003 & 4817 & 3820 & 68 \\
\hline $\mathrm{H}(65 \mathrm{~A})$ & 7239 & 3659 & 6471 & 88 \\
\hline $\mathrm{H}(65 \mathrm{~B})$ & 6283 & 3608 & 6040 & 88 \\
\hline $\mathrm{H}(65 \mathrm{C})$ & 6730 & 4200 & 6201 & 88 \\
\hline $\mathrm{H}(66 \mathrm{~A})$ & 8193 & 3023 & 5836 & 78 \\
\hline $\mathrm{H}(66 \mathrm{~B})$ & 8222 & 3162 & 5171 & 78 \\
\hline $\mathrm{H}(66 \mathrm{C})$ & 7214 & 2965 & 5426 & 78 \\
\hline $\mathrm{H}(67 \mathrm{~A})$ & 8770 & 3918 & 6190 & 84 \\
\hline $\mathrm{H}(67 \mathrm{~B})$ & 8396 & 4464 & 5866 & 84 \\
\hline $\mathrm{H}(67 \mathrm{C})$ & 9010 & 4015 & 5539 & 84 \\
\hline $\mathrm{H}(69 \mathrm{~A})$ & 8762 & 5427 & 4602 & 164 \\
\hline $\mathrm{H}(69 \mathrm{~B})$ & 9326 & 5392 & 4024 & 164 \\
\hline $\mathrm{H}(69 \mathrm{C})$ & 9233 & 4857 & 4413 & 164 \\
\hline $\mathrm{H}(70 \mathrm{~A})$ & 7175 & 5774 & 4021 & 165 \\
\hline $\mathrm{H}(70 \mathrm{~B})$ & 6818 & 5429 & 3465 & 165 \\
\hline $\mathrm{H}(70 \mathrm{C})$ & 7833 & 5763 & 3478 & 165 \\
\hline $\mathrm{H}(71 \mathrm{~A})$ & 7665 & 4597 & 3195 & 125 \\
\hline $\mathrm{H}(71 \mathrm{~B})$ & 8597 & 4368 & 3573 & 125 \\
\hline $\mathrm{H}(71 \mathrm{C})$ & 8693 & 4918 & 3207 & 125 \\
\hline
\end{tabular}




\begin{tabular}{lllll}
$\mathrm{H}(73 \mathrm{~A})$ & 4613 & 4855 & 3586 & 191 \\
$\mathrm{H}(73 \mathrm{~B})$ & 4474 & 5148 & 4186 & 191 \\
$\mathrm{H}(73 \mathrm{C})$ & 3555 & 4857 & 3851 & 191 \\
$\mathrm{H}(74 \mathrm{~A})$ & 3198 & 4354 & 4645 & 155 \\
$\mathrm{H}(74 \mathrm{~B})$ & 4120 & 4559 & 5047 & 155 \\
$\mathrm{H}(74 \mathrm{C})$ & 3938 & 3916 & 4947 & 155 \\
$\mathrm{H}(75 \mathrm{~A})$ & 4635 & 3834 & 3510 & 97 \\
$\mathrm{H}(75 \mathrm{~B})$ & 3522 & 3890 & 3690 & 97 \\
$\mathrm{H}(75 \mathrm{C})$ & 4267 & 3478 & 4030 & 97 \\
\hline
\end{tabular}




\section{Data for 2a}

Table S6. Crystal data and structure refinement for cain07_sq (2a).

Identification code

cain07_sq

Empirical formula

C76 H103 Cl4 P4 Rh

Formula weight

1385.17

Temperature

$100.0 \mathrm{~K}$

Wavelength

$0.71073 \AA$

Crystal system

Trigonal

Space group

Unit cell dimensions

R -3

$\mathrm{a}=31.949(3) \AA \quad \alpha=90^{\circ}$.

$\mathrm{b}=31.949(3) \AA \quad \beta=90^{\circ}$.

$\mathrm{c}=42.298(4) \AA \quad \gamma=120^{\circ}$.

Volume

37391(8) $\AA^{3}$

Z

18

Density (calculated)

$1.107 \mathrm{Mg} / \mathrm{m}^{3}$

Absorption coefficient

$0.447 \mathrm{~mm}^{-1}$

$\mathrm{F}(000)$

13176

Crystal size

$0.3 \times 0.27 \times 0.23 \mathrm{~mm}^{3}$

Theta range for data collection

1.212 to $28.280^{\circ}$.

Index ranges

$-31<=\mathrm{h}<=31,-11<=\mathrm{k}<=42,-56<=\mathrm{l}<=37$

Reflections collected

30316

Independent reflections

$19854[\mathrm{R}(\mathrm{int})=0.0735]$

Completeness to theta $=25.000^{\circ}$

$96.9 \%$

Absorption correction

Semi-empirical from equivalents

Max. and min. transmission

0.203 and 0.156

Refinement method

Full-matrix least-squares on $\mathrm{F}^{2}$

Data / restraints / parameters

19854 / 36 / 824

Goodness-of-fit on $\mathrm{F}^{2}$

0.882

Final $R$ indices [I $>2 \operatorname{sigma}(\mathrm{I})]$

$\mathrm{R} 1=0.0602, \mathrm{wR} 2=0.1368$

$\mathrm{R}$ indices (all data)

$\mathrm{R} 1=0.1138, \mathrm{wR} 2=0.1625$

Extinction coefficient

$\mathrm{n} / \mathrm{a}$

Largest diff. peak and hole

1.060 and $-1.014 \mathrm{e} . \AA^{-3}$

SQUEEZE

2475e/uc;calc'd for $54 \mathrm{CHCl}_{3}: 3132 \mathrm{e} / \mathrm{uc}$ 
Table S7. Atomic coordinates ( $\left.\mathrm{x} 10^{4}\right)$ and equivalent isotropic displacement parameters $\left(\AA^{2} \times 10^{3}\right)$ for cain07_sq. U(eq) is defined as one third of the trace of the orthogonalized $\mathrm{U}^{\mathrm{ij}}$ tensor.

\begin{tabular}{|c|c|c|c|c|}
\hline & $\mathrm{x}$ & $\mathrm{y}$ & $\mathrm{z}$ & $\mathrm{U}(\mathrm{eq})$ \\
\hline $\operatorname{Rh}(1)$ & $3303(1)$ & $3337(1)$ & $6378(1)$ & $15(1)$ \\
\hline $\mathrm{Cl}(1)$ & $3338(1)$ & $3441(1)$ & $5810(1)$ & $29(1)$ \\
\hline $\mathrm{Cl}(2)$ & $3403(1)$ & $2866(1)$ & $4880(1)$ & $79(1)$ \\
\hline $\mathrm{Cl}(3)$ & $2680(1)$ & $3157(1)$ & $4860(1)$ & $76(1)$ \\
\hline $\mathrm{Cl}(4)$ & $3688(1)$ & $3866(1)$ & $4786(1)$ & $67(1)$ \\
\hline $\mathrm{P}(1)$ & $3298(1)$ & $3304(1)$ & $6902(1)$ & $15(1)$ \\
\hline $\mathrm{P}(2)$ & $4080(1)$ & $3943(1)$ & $6418(1)$ & $17(1)$ \\
\hline $\mathrm{P}(3)$ & $2674(1)$ & $3476(1)$ & $6416(1)$ & $17(1)$ \\
\hline $\mathrm{P}(4)$ & $3145(1)$ & 2551(1) & $6379(1)$ & $18(1)$ \\
\hline $\mathrm{C}(1)$ & $4796(1)$ & 4333(1) & $5575(1)$ & $27(1)$ \\
\hline$C(2)$ & $4558(1)$ & $4367(1)$ & $5842(1)$ & $22(1)$ \\
\hline$C(3)$ & $4563(1)$ & $4117(1)$ & $6121(1)$ & $18(1)$ \\
\hline $\mathrm{C}(4)$ & $4866(1)$ & 3899(1) & $6128(1)$ & $20(1)$ \\
\hline$C(5)$ & $5082(1)$ & $3885(1)$ & $5846(1)$ & $23(1)$ \\
\hline$C(6)$ & $5034(1)$ & 4078(1) & $5562(1)$ & $27(1)$ \\
\hline$C(7)$ & $4369(1)$ & $4725(1)$ & $5801(1)$ & $24(1)$ \\
\hline $\mathrm{C}(8)$ & $4052(1)$ & $4747(1)$ & $6068(1)$ & $36(1)$ \\
\hline$C(9)$ & $4810(1)$ & $5236(1)$ & $5779(1)$ & $40(1)$ \\
\hline$C(10)$ & $4062(2)$ & $4623(2)$ & $5504(1)$ & $42(1)$ \\
\hline $\mathrm{C}(11)$ & 4994(1) & $3700(1)$ & $6424(1)$ & $24(1)$ \\
\hline$C(12)$ & $5223(2)$ & 4081(1) & $6677(1)$ & $40(1)$ \\
\hline$C(13)$ & $4553(1)$ & $3248(1)$ & $6554(1)$ & $35(1)$ \\
\hline$C(14)$ & $5372(2)$ & $3542(2)$ & $6350(1)$ & $45(1)$ \\
\hline$C(15)$ & $5243(1)$ & 4012(2) & $5252(1)$ & $35(1)$ \\
\hline$C(16)$ & $5520(3)$ & $3713(3)$ & $5300(2)$ & $63(2)$ \\
\hline$C(17)$ & $4857(3)$ & $3737(3)$ & $5017(2)$ & $53(2)$ \\
\hline $\mathrm{C}(18)$ & $5621(3)$ & 4487(3) & $5117(2)$ & $60(2)$ \\
\hline$C(19)$ & $2806(1)$ & 2057(1) & $6090(1)$ & $20(1)$ \\
\hline $\mathrm{C}(20)$ & 2293(1) & $1756(1)$ & $6109(1)$ & 19(1) \\
\hline $\mathrm{C}(21)$ & $2040(1)$ & 1523(1) & $5833(1)$ & $22(1)$ \\
\hline $\mathrm{C}(22)$ & 2264(1) & 1561(1) & $5546(1)$ & $22(1)$ \\
\hline
\end{tabular}




\begin{tabular}{|c|c|c|c|c|}
\hline $\mathrm{C}(23)$ & $2765(1)$ & 1804(1) & $5546(1)$ & $23(1)$ \\
\hline$C(24)$ & $3051(1)$ & 2046(1) & $5804(1)$ & $20(1)$ \\
\hline$C(25)$ & 1981(1) & $1639(1)$ & $6414(1)$ & $23(1)$ \\
\hline$C(26)$ & 1438(1) & $1273(2)$ & $6352(1)$ & $46(1)$ \\
\hline$C(27)$ & $1985(1)$ & 2084(1) & $6546(1)$ & $37(1)$ \\
\hline $\mathrm{C}(28)$ & $2140(2)$ & 1402(2) & $6665(1)$ & $44(1)$ \\
\hline$C(29)$ & 1977(1) & 1319(1) & $5243(1)$ & $30(1)$ \\
\hline$C(30)$ & $1426(1)$ & $1108(2)$ & $5290(1)$ & $45(1)$ \\
\hline $\mathrm{C}(31)$ & $2059(2)$ & $904(1)$ & $5152(1)$ & $42(1)$ \\
\hline $\mathrm{C}(32)$ & $2148(1)$ & 1694(2) & $4978(1)$ & $37(1)$ \\
\hline $\mathrm{C}(33)$ & $3605(1)$ & $2250(1)$ & $5770(1)$ & $24(1)$ \\
\hline$C(34)$ & $3733(1)$ & $2075(2)$ & $5466(1)$ & $45(1)$ \\
\hline$C(35)$ & $3790(1)$ & 2046(1) & $6039(1)$ & $33(1)$ \\
\hline$C(36)$ & $3899(1)$ & 2794(1) & $5768(1)$ & $40(1)$ \\
\hline $\mathrm{C}(37)$ & $2810(1)$ & $4604(1)$ & $5881(1)$ & $21(1)$ \\
\hline $\mathrm{C}(38)$ & $2655(1)$ & 4395(1) & $5585(1)$ & $23(1)$ \\
\hline C(39) & $2380(1)$ & $3896(1)$ & $5576(1)$ & $24(1)$ \\
\hline $\mathrm{C}(40)$ & 2297(1) & $3596(1)$ & $5837(1)$ & $20(1)$ \\
\hline $\mathrm{C}(41)$ & $2528(1)$ & $3826(1)$ & 6131(1) & $20(1)$ \\
\hline$C(42)$ & 2751(1) & 4339(1) & $6158(1)$ & $20(1)$ \\
\hline $\mathrm{C}(43)$ & $2748(1)$ & $4697(1)$ & $5284(1)$ & $27(1)$ \\
\hline C(44) & $2927(1)$ & 4497(1) & $5020(1)$ & $35(1)$ \\
\hline $\mathrm{C}(45)$ & $2278(1)$ & $4674(2)$ & $5179(1)$ & $38(1)$ \\
\hline $\mathrm{C}(46)$ & $3136(2)$ & $5234(1)$ & $5336(1)$ & $41(1)$ \\
\hline$C(47)$ & 1934(1) & $3048(1)$ & $5792(1)$ & $24(1)$ \\
\hline $\mathrm{C}(48)$ & $1614(2)$ & $2958(2)$ & $5503(1)$ & $50(1)$ \\
\hline C(49) & $2158(1)$ & $2738(1)$ & $5768(1)$ & $40(1)$ \\
\hline$C(50)$ & $1552(1)$ & $2863(1)$ & $6065(1)$ & $36(1)$ \\
\hline$C(51)$ & $2890(1)$ & $4634(1)$ & $6472(1)$ & $22(1)$ \\
\hline $\mathrm{C}(52)$ & $2428(1)$ & 4481(1) & $6665(1)$ & $32(1)$ \\
\hline$C(53)$ & $3249(1)$ & $4570(1)$ & 6681(1) & $28(1)$ \\
\hline$C(54)$ & $3122(2)$ & $5187(1)$ & $6418(1)$ & $34(1)$ \\
\hline$C(55)$ & 2704(1) & 2944(1) & $7079(1)$ & $18(1)$ \\
\hline$C(56)$ & $2638(1)$ & 2612(1) & $7315(1)$ & $24(1)$ \\
\hline$C(57)$ & $2200(1)$ & $2343(1)$ & $7469(1)$ & $33(1)$ \\
\hline C(58) & 1821(1) & 2418(1) & 7396(1) & $29(1)$ \\
\hline
\end{tabular}




\begin{tabular}{lllll}
$\mathrm{C}(59)$ & $1878(1)$ & $2747(1)$ & $7163(1)$ & $23(1)$ \\
$\mathrm{C}(60)$ & $2310(1)$ & $3008(1)$ & $6990(1)$ & $18(1)$ \\
$\mathrm{C}(61)$ & $2314(1)$ & $3310(1)$ & $6733(1)$ & $18(1)$ \\
$\mathrm{C}(62)$ & $3545(1)$ & $3886(1)$ & $7101(1)$ & $18(1)$ \\
$\mathrm{C}(63)$ & $3291(1)$ & $3925(1)$ & $7360(1)$ & $23(1)$ \\
$\mathrm{C}(64)$ & $3474(1)$ & $4349(1)$ & $7533(1)$ & $28(1)$ \\
$\mathrm{C}(65)$ & $3919(1)$ & $4742(1)$ & $7446(1)$ & $30(1)$ \\
$\mathrm{C}(66)$ & $4170(1)$ & $4708(1)$ & $7192(1)$ & $23(1)$ \\
$\mathrm{C}(67)$ & $3990(1)$ & $4288(1)$ & $7007(1)$ & $18(1)$ \\
$\mathrm{C}(68)$ & $4278(1)$ & $4298(1)$ & $6738(1)$ & $19(1)$ \\
$\mathrm{C}(69)$ & $3652(1)$ & $3052(1)$ & $7069(1)$ & $17(1)$ \\
$\mathrm{C}(70)$ & $3964(1)$ & $3290(1)$ & $7321(1)$ & $24(1)$ \\
$\mathrm{C}(71)$ & $4224(1)$ & $3103(1)$ & $7468(1)$ & $29(1)$ \\
$\mathrm{C}(72)$ & $4164(1)$ & $2663(1)$ & $7364(1)$ & $29(1)$ \\
$\mathrm{C}(73)$ & $3854(1)$ & $2422(1)$ & $7118(1)$ & $24(1)$ \\
$\mathrm{C}(74)$ & $3598(1)$ & $2612(1)$ & $6958(1)$ & $18(1)$ \\
$\mathrm{C}(75)$ & $3307(1)$ & $2339(1)$ & $6689(1)$ & $21(1)$ \\
$\mathrm{C}(81)$ & $3271(2)$ & $3324(2)$ & $4975(1)$ & $58(2)$ \\
$\mathrm{C}(16 \mathrm{~A})$ & $5015(5)$ & $3503(4)$ & $5179(3)$ & $50(3)$ \\
$\mathrm{C}(17 \mathrm{~A})$ & $5070(5)$ & $4234(5)$ & $4962(3)$ & $53(3)$ \\
$\mathrm{C}(18 \mathrm{~A})$ & $5775(4)$ & $4273(5)$ & $5262(4)$ & $48(3)$ \\
\hline
\end{tabular}


Table S8. Bond lengths $[\AA]$ and angles $\left[^{\circ}\right]$ for cain07_sq.

\begin{tabular}{|c|c|}
\hline $\mathrm{Rh}(1)-\mathrm{Cl}(1)$ & $2.4204(11)$ \\
\hline $\mathrm{Rh}(1)-\mathrm{P}(1)$ & $2.2201(10)$ \\
\hline $\mathrm{Rh}(1)-\mathrm{P}(2)$ & $2.2673(9)$ \\
\hline $\mathrm{Rh}(1)-\mathrm{P}(3)$ & $2.2690(9)$ \\
\hline $\mathrm{Rh}(1)-\mathrm{P}(4)$ & $2.3026(9)$ \\
\hline $\mathrm{Cl}(2)-\mathrm{C}(81)$ & $1.761(5)$ \\
\hline $\mathrm{Cl}(3)-\mathrm{C}(81)$ & $1.754(5)$ \\
\hline $\mathrm{Cl}(4)-\mathrm{C}(81)$ & $1.760(5)$ \\
\hline$P(1)-C(55)$ & $1.817(3)$ \\
\hline$P(1)-C(62)$ & $1.820(3)$ \\
\hline $\mathrm{P}(1)-\mathrm{C}(69)$ & $1.826(3)$ \\
\hline $\mathrm{P}(2)-\mathrm{C}(3)$ & $1.846(4)$ \\
\hline $\mathrm{P}(2)-\mathrm{C}(68)$ & $1.673(4)$ \\
\hline $\mathrm{P}(3)-\mathrm{C}(41)$ & $1.854(4)$ \\
\hline $\mathrm{P}(3)-\mathrm{C}(61)$ & $1.673(4)$ \\
\hline $\mathrm{P}(4)-\mathrm{C}(19)$ & $1.858(4)$ \\
\hline$P(4)-C(75)$ & $1.673(4)$ \\
\hline $\mathrm{C}(1)-\mathrm{C}(2)$ & $1.396(5)$ \\
\hline$C(1)-C(6)$ & $1.364(5)$ \\
\hline $\mathrm{C}(2)-\mathrm{C}(3)$ & $1.432(5)$ \\
\hline $\mathrm{C}(2)-\mathrm{C}(7)$ & $1.547(5)$ \\
\hline $\mathrm{C}(3)-\mathrm{C}(4)$ & $1.448(5)$ \\
\hline $\mathrm{C}(4)-\mathrm{C}(5)$ & $1.393(5)$ \\
\hline $\mathrm{C}(4)-\mathrm{C}(11)$ & $1.550(5)$ \\
\hline$C(5)-C(6)$ & $1.394(5)$ \\
\hline$C(6)-C(15)$ & $1.532(6)$ \\
\hline$C(7)-C(8)$ & $1.539(5)$ \\
\hline $\mathrm{C}(7)-\mathrm{C}(9)$ & $1.536(5)$ \\
\hline$C(7)-C(10)$ & $1.526(5)$ \\
\hline $\mathrm{C}(11)-\mathrm{C}(12)$ & $1.508(5)$ \\
\hline $\mathrm{C}(11)-\mathrm{C}(13)$ & $1.530(5)$ \\
\hline $\mathrm{C}(11)-\mathrm{C}(14)$ & $1.555(5)$ \\
\hline$C(15)-C(16)$ & $1.605(8)$ \\
\hline$C(15)-C(17)$ & $1.482(8)$ \\
\hline
\end{tabular}




\begin{tabular}{|c|c|}
\hline$C(15)-C(18)$ & $1.502(8)$ \\
\hline$C(15)-C(16 A)$ & $1.443(11)$ \\
\hline$C(15)-C(17 A)$ & $1.647(13)$ \\
\hline $\mathrm{C}(15)-\mathrm{C}(18 \mathrm{~A})$ & $1.471(12)$ \\
\hline C(19)-C(20) & $1.429(4)$ \\
\hline C(19)-C(24) & $1.451(5)$ \\
\hline$C(20)-C(21)$ & $1.402(5)$ \\
\hline$C(20)-C(25)$ & $1.555(5)$ \\
\hline$C(21)-C(22)$ & $1.384(5)$ \\
\hline$C(22)-C(23)$ & $1.388(5)$ \\
\hline$C(22)-C(29)$ & $1.538(5)$ \\
\hline$C(23)-C(24)$ & $1.384(5)$ \\
\hline$C(24)-C(33)$ & $1.557(5)$ \\
\hline$C(25)-C(26)$ & $1.554(5)$ \\
\hline$C(25)-C(27)$ & $1.520(5)$ \\
\hline$C(25)-C(28)$ & $1.532(5)$ \\
\hline$C(29)-C(30)$ & $1.551(5)$ \\
\hline $\mathrm{C}(29)-\mathrm{C}(31)$ & $1.526(5)$ \\
\hline C(29)-C(32) & $1.528(5)$ \\
\hline $\mathrm{C}(33)-\mathrm{C}(34)$ & $1.536(6)$ \\
\hline C(33)-C(35) & $1.562(5)$ \\
\hline C(33)-C(36) & $1.509(5)$ \\
\hline C(37)-C(38) & $1.387(5)$ \\
\hline $\mathrm{C}(37)-\mathrm{C}(42)$ & $1.400(5)$ \\
\hline C(38)-C(39) & $1.382(5)$ \\
\hline C(38)-C(43) & $1.536(5)$ \\
\hline C(39)-C(40) & $1.397(5)$ \\
\hline $\mathrm{C}(40)-\mathrm{C}(41)$ & $1.443(5)$ \\
\hline $\mathrm{C}(40)-\mathrm{C}(47)$ & $1.556(5)$ \\
\hline $\mathrm{C}(41)-\mathrm{C}(42)$ & $1.429(5)$ \\
\hline $\mathrm{C}(42)-\mathrm{C}(51)$ & $1.561(5)$ \\
\hline $\mathrm{C}(43)-\mathrm{C}(44)$ & $1.533(5)$ \\
\hline $\mathrm{C}(43)-\mathrm{C}(45)$ & $1.532(5)$ \\
\hline$C(43)-C(46)$ & $1.551(5)$ \\
\hline $\mathrm{C}(47)-\mathrm{C}(48)$ & $1.524(5)$ \\
\hline C(47)-C(49) & $1.489(5)$ \\
\hline
\end{tabular}




\begin{tabular}{|c|c|}
\hline $\mathrm{C}(47)-\mathrm{C}(50)$ & $1.566(5)$ \\
\hline $\mathrm{C}(51)-\mathrm{C}(52)$ & $1.538(5)$ \\
\hline$C(51)-C(53)$ & $1.538(5)$ \\
\hline $\mathrm{C}(51)-\mathrm{C}(54)$ & $1.555(5)$ \\
\hline C(55)-C(56) & $1.392(5)$ \\
\hline$C(55)-C(60)$ & $1.426(4)$ \\
\hline$C(56)-C(57)$ & $1.386(5)$ \\
\hline$C(57)-C(58)$ & $1.381(5)$ \\
\hline C(58)-C(59) & $1.383(5)$ \\
\hline C(59)-C(60) & $1.407(5)$ \\
\hline $\mathrm{C}(60)-\mathrm{C}(61)$ & $1.450(5)$ \\
\hline$C(62)-C(63)$ & $1.404(5)$ \\
\hline$C(62)-C(67)$ & $1.416(5)$ \\
\hline$C(63)-C(64)$ & $1.386(5)$ \\
\hline $\mathrm{C}(64)-\mathrm{C}(65)$ & $1.396(5)$ \\
\hline $\mathrm{C}(65)-\mathrm{C}(66)$ & $1.376(5)$ \\
\hline $\mathrm{C}(66)-\mathrm{C}(67)$ & $1.404(5)$ \\
\hline $\mathrm{C}(67)-\mathrm{C}(68)$ & $1.454(5)$ \\
\hline $\mathrm{C}(69)-\mathrm{C}(70)$ & $1.398(5)$ \\
\hline C(69)-C(74) & $1.409(4)$ \\
\hline$C(70)-C(71)$ & $1.387(5)$ \\
\hline $\mathrm{C}(71)-\mathrm{C}(72)$ & $1.392(5)$ \\
\hline$C(72)-C(73)$ & $1.378(5)$ \\
\hline$C(73)-C(74)$ & $1.410(5)$ \\
\hline$C(74)-C(75)$ & $1.452(5)$ \\
\hline $\mathrm{P}(1)-\mathrm{Rh}(1)-\mathrm{Cl}(1)$ & $175.53(3)$ \\
\hline $\mathrm{P}(1)-\mathrm{Rh}(1)-\mathrm{P}(2)$ & $86.73(4)$ \\
\hline $\mathrm{P}(1)-\mathrm{Rh}(1)-\mathrm{P}(3)$ & $87.36(4)$ \\
\hline $\mathrm{P}(1)-\mathrm{Rh}(1)-\mathrm{P}(4)$ & $87.36(4)$ \\
\hline $\mathrm{P}(2)-\mathrm{Rh}(1)-\mathrm{Cl}(1)$ & $90.09(3)$ \\
\hline $\mathrm{P}(2)-\mathrm{Rh}(1)-\mathrm{P}(3)$ & $121.81(3)$ \\
\hline $\mathrm{P}(2)-\mathrm{Rh}(1)-\mathrm{P}(4)$ & $118.66(3)$ \\
\hline $\mathrm{P}(3)-\mathrm{Rh}(1)-\mathrm{Cl}(1)$ & $91.67(3)$ \\
\hline $\mathrm{P}(3)-\mathrm{Rh}(1)-\mathrm{P}(4)$ & $118.79(3)$ \\
\hline $\mathrm{P}(4)-\mathrm{Rh}(1)-\mathrm{Cl}(1)$ & 96.92(4) \\
\hline
\end{tabular}




\begin{tabular}{|c|c|}
\hline $\mathrm{C}(55)-\mathrm{P}(1)-\mathrm{Rh}(1)$ & $114.87(13)$ \\
\hline $\mathrm{C}(55)-\mathrm{P}(1)-\mathrm{C}(62)$ & $103.84(16)$ \\
\hline $\mathrm{C}(55)-\mathrm{P}(1)-\mathrm{C}(69)$ & $103.91(16)$ \\
\hline $\mathrm{C}(62)-\mathrm{P}(1)-\mathrm{Rh}(1)$ & $115.13(12)$ \\
\hline $\mathrm{C}(62)-\mathrm{P}(1)-\mathrm{C}(69)$ & $102.93(16)$ \\
\hline $\mathrm{C}(69)-\mathrm{P}(1)-\mathrm{Rh}(1)$ & $114.63(13)$ \\
\hline $\mathrm{C}(3)-\mathrm{P}(2)-\mathrm{Rh}(1)$ & $126.80(12)$ \\
\hline $\mathrm{C}(68)-\mathrm{P}(2)-\mathrm{Rh}(1)$ & $121.39(13)$ \\
\hline $\mathrm{C}(68)-\mathrm{P}(2)-\mathrm{C}(3)$ & $111.80(17)$ \\
\hline $\mathrm{C}(41)-\mathrm{P}(3)-\mathrm{Rh}(1)$ & $125.83(12)$ \\
\hline $\mathrm{C}(61)-\mathrm{P}(3)-\mathrm{Rh}(1)$ & $122.02(13)$ \\
\hline $\mathrm{C}(61)-\mathrm{P}(3)-\mathrm{C}(41)$ & 111.91(17) \\
\hline $\mathrm{C}(19)-\mathrm{P}(4)-\mathrm{Rh}(1)$ & $129.95(12)$ \\
\hline $\mathrm{C}(75)-\mathrm{P}(4)-\mathrm{Rh}(1)$ & $119.99(13)$ \\
\hline $\mathrm{C}(75)-\mathrm{P}(4)-\mathrm{C}(19)$ & $109.88(17)$ \\
\hline $\mathrm{C}(6)-\mathrm{C}(1)-\mathrm{C}(2)$ & 124.7(4) \\
\hline $\mathrm{C}(1)-\mathrm{C}(2)-\mathrm{C}(3)$ & $117.7(3)$ \\
\hline $\mathrm{C}(1)-\mathrm{C}(2)-\mathrm{C}(7)$ & $113.5(3)$ \\
\hline $\mathrm{C}(3)-\mathrm{C}(2)-\mathrm{C}(7)$ & $128.4(3)$ \\
\hline $\mathrm{C}(2)-\mathrm{C}(3)-\mathrm{P}(2)$ & 119.3(3) \\
\hline $\mathrm{C}(2)-\mathrm{C}(3)-\mathrm{C}(4)$ & $118.8(3)$ \\
\hline $\mathrm{C}(4)-\mathrm{C}(3)-\mathrm{P}(2)$ & $120.0(3)$ \\
\hline $\mathrm{C}(3)-\mathrm{C}(4)-\mathrm{C}(11)$ & $126.3(3)$ \\
\hline $\mathrm{C}(5)-\mathrm{C}(4)-\mathrm{C}(3)$ & $117.4(3)$ \\
\hline $\mathrm{C}(5)-\mathrm{C}(4)-\mathrm{C}(11)$ & $116.2(3)$ \\
\hline$C(4)-C(5)-C(6)$ & $124.0(4)$ \\
\hline$C(1)-C(6)-C(5)$ & $116.5(4)$ \\
\hline$C(1)-C(6)-C(15)$ & $122.0(4)$ \\
\hline$C(5)-C(6)-C(15)$ & $121.6(4)$ \\
\hline $\mathrm{C}(8)-\mathrm{C}(7)-\mathrm{C}(2)$ & $117.5(3)$ \\
\hline $\mathrm{C}(9)-\mathrm{C}(7)-\mathrm{C}(2)$ & 107.6(3) \\
\hline $\mathrm{C}(9)-\mathrm{C}(7)-\mathrm{C}(8)$ & $105.8(3)$ \\
\hline $\mathrm{C}(10)-\mathrm{C}(7)-\mathrm{C}(2)$ & $112.4(3)$ \\
\hline $\mathrm{C}(10)-\mathrm{C}(7)-\mathrm{C}(8)$ & $104.2(3)$ \\
\hline $\mathrm{C}(10)-\mathrm{C}(7)-\mathrm{C}(9)$ & $109.0(3)$ \\
\hline $\mathrm{C}(4)-\mathrm{C}(11)-\mathrm{C}(14)$ & $112.2(3)$ \\
\hline
\end{tabular}




\begin{tabular}{|c|c|}
\hline $\mathrm{C}(12)-\mathrm{C}(11)-\mathrm{C}(4)$ & $111.2(3)$ \\
\hline $\mathrm{C}(12)-\mathrm{C}(11)-\mathrm{C}(13)$ & $110.7(4)$ \\
\hline$C(12)-C(11)-C(14)$ & $105.3(3)$ \\
\hline $\mathrm{C}(13)-\mathrm{C}(11)-\mathrm{C}(4)$ & $111.7(3)$ \\
\hline$C(13)-C(11)-C(14)$ & $105.4(3)$ \\
\hline $\mathrm{C}(6)-\mathrm{C}(15)-\mathrm{C}(16)$ & $112.5(5)$ \\
\hline$C(6)-C(15)-C(17 A)$ & $108.3(5)$ \\
\hline$C(17)-C(15)-C(6)$ & $111.2(4)$ \\
\hline$C(17)-C(15)-C(16)$ & $106.1(5)$ \\
\hline $\mathrm{C}(17)-\mathrm{C}(15)-\mathrm{C}(18)$ & $110.7(6)$ \\
\hline $\mathrm{C}(18)-\mathrm{C}(15)-\mathrm{C}(6)$ & $111.7(4)$ \\
\hline $\mathrm{C}(18)-\mathrm{C}(15)-\mathrm{C}(16)$ & $104.2(5)$ \\
\hline$C(16 A)-C(15)-C(6)$ & $109.1(6)$ \\
\hline$C(16 A)-C(15)-C(17 A)$ & $103.8(8)$ \\
\hline$C(16 A)-C(15)-C(18 A)$ & $115.8(8)$ \\
\hline $\mathrm{C}(18 \mathrm{~A})-\mathrm{C}(15)-\mathrm{C}(6)$ & $110.8(6)$ \\
\hline $\mathrm{C}(18 \mathrm{~A})-\mathrm{C}(15)-\mathrm{C}(17 \mathrm{~A})$ & $108.6(8)$ \\
\hline $\mathrm{C}(20)-\mathrm{C}(19)-\mathrm{P}(4)$ & $121.3(3)$ \\
\hline $\mathrm{C}(20)-\mathrm{C}(19)-\mathrm{C}(24)$ & $118.9(3)$ \\
\hline $\mathrm{C}(24)-\mathrm{C}(19)-\mathrm{P}(4)$ & $118.5(3)$ \\
\hline$C(19)-C(20)-C(25)$ & $126.2(3)$ \\
\hline$C(21)-C(20)-C(19)$ & $118.2(3)$ \\
\hline$C(21)-C(20)-C(25)$ & $115.6(3)$ \\
\hline$C(22)-C(21)-C(20)$ & $123.3(3)$ \\
\hline$C(21)-C(22)-C(23)$ & $116.8(4)$ \\
\hline $\mathrm{C}(21)-\mathrm{C}(22)-\mathrm{C}(29)$ & $122.3(3)$ \\
\hline$C(23)-C(22)-C(29)$ & $120.7(4)$ \\
\hline $\mathrm{C}(24)-\mathrm{C}(23)-\mathrm{C}(22)$ & $124.5(4)$ \\
\hline$C(19)-C(24)-C(33)$ & $125.5(3)$ \\
\hline$C(23)-C(24)-C(19)$ & $117.2(3)$ \\
\hline $\mathrm{C}(23)-\mathrm{C}(24)-\mathrm{C}(33)$ & $117.2(3)$ \\
\hline$C(26)-C(25)-C(20)$ & $112.4(3)$ \\
\hline$C(27)-C(25)-C(20)$ & $112.1(3)$ \\
\hline$C(27)-C(25)-C(26)$ & $104.4(3)$ \\
\hline$C(27)-C(25)-C(28)$ & $110.9(4)$ \\
\hline$C(28)-C(25)-C(20)$ & $111.3(3)$ \\
\hline
\end{tabular}




\begin{tabular}{|c|c|}
\hline $\mathrm{C}(28)-\mathrm{C}(25)-\mathrm{C}(26)$ & $105.4(3)$ \\
\hline $\mathrm{C}(22)-\mathrm{C}(29)-\mathrm{C}(30)$ & $112.0(4)$ \\
\hline$C(31)-C(29)-C(22)$ & 109.3(3) \\
\hline $\mathrm{C}(31)-\mathrm{C}(29)-\mathrm{C}(30)$ & $107.8(3)$ \\
\hline $\mathrm{C}(31)-\mathrm{C}(29)-\mathrm{C}(32)$ & $110.2(4)$ \\
\hline $\mathrm{C}(32)-\mathrm{C}(29)-\mathrm{C}(22)$ & 109.1(3) \\
\hline $\mathrm{C}(32)-\mathrm{C}(29)-\mathrm{C}(30)$ & $108.5(3)$ \\
\hline$C(24)-C(33)-C(35)$ & $110.2(3)$ \\
\hline $\mathrm{C}(34)-\mathrm{C}(33)-\mathrm{C}(24)$ & 112.1(3) \\
\hline $\mathrm{C}(34)-\mathrm{C}(33)-\mathrm{C}(35)$ & $103.6(3)$ \\
\hline $\mathrm{C}(36)-\mathrm{C}(33)-\mathrm{C}(24)$ & $113.8(3)$ \\
\hline $\mathrm{C}(36)-\mathrm{C}(33)-\mathrm{C}(34)$ & $106.9(3)$ \\
\hline $\mathrm{C}(36)-\mathrm{C}(33)-\mathrm{C}(35)$ & $109.7(3)$ \\
\hline $\mathrm{C}(38)-\mathrm{C}(37)-\mathrm{C}(42)$ & $123.7(3)$ \\
\hline $\mathrm{C}(37)-\mathrm{C}(38)-\mathrm{C}(43)$ & $122.4(3)$ \\
\hline $\mathrm{C}(39)-\mathrm{C}(38)-\mathrm{C}(37)$ & $116.6(4)$ \\
\hline $\mathrm{C}(39)-\mathrm{C}(38)-\mathrm{C}(43)$ & $120.8(4)$ \\
\hline $\mathrm{C}(38)-\mathrm{C}(39)-\mathrm{C}(40)$ & $124.1(4)$ \\
\hline$C(39)-C(40)-C(41)$ & $117.3(3)$ \\
\hline$C(39)-C(40)-C(47)$ & $116.9(3)$ \\
\hline $\mathrm{C}(41)-\mathrm{C}(40)-\mathrm{C}(47)$ & $125.7(3)$ \\
\hline $\mathrm{C}(40)-\mathrm{C}(41)-\mathrm{P}(3)$ & $118.2(3)$ \\
\hline $\mathrm{C}(42)-\mathrm{C}(41)-\mathrm{P}(3)$ & $120.6(3)$ \\
\hline$C(42)-C(41)-C(40)$ & 119.1(3) \\
\hline$C(37)-C(42)-C(41)$ & $117.8(4)$ \\
\hline $\mathrm{C}(37)-\mathrm{C}(42)-\mathrm{C}(51)$ & $115.8(3)$ \\
\hline$C(41)-C(42)-C(51)$ & $126.1(3)$ \\
\hline$C(38)-C(43)-C(46)$ & $112.0(4)$ \\
\hline $\mathrm{C}(44)-\mathrm{C}(43)-\mathrm{C}(38)$ & $109.8(3)$ \\
\hline$C(44)-C(43)-C(46)$ & $107.8(3)$ \\
\hline $\mathrm{C}(45)-\mathrm{C}(43)-\mathrm{C}(38)$ & $109.5(3)$ \\
\hline$C(45)-C(43)-C(44)$ & 109.2(4) \\
\hline$C(45)-C(43)-C(46)$ & $108.4(3)$ \\
\hline$C(40)-C(47)-C(50)$ & $109.5(3)$ \\
\hline $\mathrm{C}(48)-\mathrm{C}(47)-\mathrm{C}(40)$ & $111.3(3)$ \\
\hline $\mathrm{C}(48)-\mathrm{C}(47)-\mathrm{C}(50)$ & $101.4(3)$ \\
\hline
\end{tabular}




$\begin{array}{ll}\mathrm{C}(49)-\mathrm{C}(47)-\mathrm{C}(40) & 115.0(3) \\ \mathrm{C}(49)-\mathrm{C}(47)-\mathrm{C}(48) & 108.7(4) \\ \mathrm{C}(49)-\mathrm{C}(47)-\mathrm{C}(50) & 110.0(3) \\ \mathrm{C}(52)-\mathrm{C}(51)-\mathrm{C}(42) & 108.7(3) \\ \mathrm{C}(52)-\mathrm{C}(51)-\mathrm{C}(54) & 106.0(3) \\ \mathrm{C}(53)-\mathrm{C}(51)-\mathrm{C}(42) & 114.5(3) \\ \mathrm{C}(53)-\mathrm{C}(51)-\mathrm{C}(52) & 108.4(4) \\ \mathrm{C}(53)-\mathrm{C}(51)-\mathrm{C}(54) & 105.8(3) \\ \mathrm{C}(54)-\mathrm{C}(51)-\mathrm{C}(42) & 113.0(3) \\ \mathrm{C}(56)-\mathrm{C}(55)-\mathrm{P}(1) & 119.4(3) \\ \mathrm{C}(56)-\mathrm{C}(55)-\mathrm{C}(60) & 119.3(3) \\ \mathrm{C}(60)-\mathrm{C}(55)-\mathrm{P}(1) & 121.3(3) \\ \mathrm{C}(57)-\mathrm{C}(56)-\mathrm{C}(55) & 122.0(3) \\ \mathrm{C}(58)-\mathrm{C}(57)-\mathrm{C}(56) & 119.2(4) \\ \mathrm{C}(57)-\mathrm{C}(58)-\mathrm{C}(59) & 119.8(3) \\ \mathrm{C}(58)-\mathrm{C}(59)-\mathrm{C}(60) & 122.5(3) \\ \mathrm{C}(55)-\mathrm{C}(60)-\mathrm{C}(61) & 125.4(3) \\ \mathrm{C}(59)-\mathrm{C}(60)-\mathrm{C}(55) & 117.0(3) \\ \mathrm{C}(59)-\mathrm{C}(60)-\mathrm{C}(61) & 117.6(3) \\ \mathrm{C}(60)-\mathrm{C}(61)-\mathrm{P}(3) & 126.1(3) \\ \mathrm{C}(63)-\mathrm{C}(62)-\mathrm{P}(1) & 118.5(3) \\ \mathrm{C}(63)-\mathrm{C}(62)-\mathrm{C}(67) & 119.9(3) \\ \mathrm{C}(67)-\mathrm{C}(62)-\mathrm{P}(1) & 121.5(3) \\ \mathrm{C}(64)-\mathrm{C}(63)-\mathrm{C}(62) & 121.3(3) \\ \mathrm{C}(63)-\mathrm{C}(64)-\mathrm{C}(65) & 118.8(4) \\ \mathrm{C}(66)-\mathrm{C}(65)-\mathrm{C}(64) & 120.4(3) \\ \mathrm{C}(65)-\mathrm{C}(66)-\mathrm{C}(67) & 122.2(4) \\ \mathrm{C}(62)-\mathrm{C}(67)-\mathrm{C}(68) & 125.2(3) \\ \mathrm{C}(66)-\mathrm{C}(67)-\mathrm{C}(62) & 117.3(3) \\ \mathrm{C}(66)-\mathrm{C}(67)-\mathrm{C}(68) & 117.5(3) \\ \mathrm{C}(67)-\mathrm{C}(68)-\mathrm{P}(2) & 126.7(3) \\ \mathrm{C}(70)-\mathrm{C}(69)-\mathrm{P}(1) & \\ \mathrm{C}(70)-\mathrm{C}(69)-\mathrm{C}(74) & \mathrm{C}(70)-\mathrm{C}(71)-\mathrm{C}(72) \\ \end{array}$




$\begin{array}{ll}\mathrm{C}(73)-\mathrm{C}(72)-\mathrm{C}(71) & 119.9(3) \\ \mathrm{C}(72)-\mathrm{C}(73)-\mathrm{C}(74) & 122.2(4) \\ \mathrm{C}(69)-\mathrm{C}(74)-\mathrm{C}(73) & 117.5(3) \\ \mathrm{C}(69)-\mathrm{C}(74)-\mathrm{C}(75) & 125.6(3) \\ \mathrm{C}(73)-\mathrm{C}(74)-\mathrm{C}(75) & 116.9(3) \\ \mathrm{C}(74)-\mathrm{C}(75)-\mathrm{P}(4) & 127.3(3) \\ \mathrm{Cl}(3)-\mathrm{C}(81)-\mathrm{Cl}(2) & 110.5(3) \\ \mathrm{Cl}(3)-\mathrm{C}(81)-\mathrm{Cl}(4) & 110.2(3) \\ \mathrm{Cl}(4)-\mathrm{C}(81)-\mathrm{Cl}(2) & 109.6(3)\end{array}$

Symmetry transformations used to generate equivalent atoms: 
Table S9. Anisotropic displacement parameters $\left(\AA^{2} \times 10^{3}\right)$ for cain07_sq. The anisotropic displacement factor exponent takes the form: $-2 \pi^{2}\left[h^{2} a^{* 2} U^{11}+\ldots+2 h \mathrm{k}^{*} b^{*} U^{12}\right]$

\begin{tabular}{|c|c|c|c|c|c|c|}
\hline & $\mathrm{U}^{11}$ & $\mathrm{U}^{22}$ & $\mathrm{U}^{33}$ & $U^{23}$ & $\mathrm{U}^{13}$ & $\mathrm{U}^{12}$ \\
\hline $\operatorname{Rh}(1)$ & $17(1)$ & $17(1)$ & $12(1)$ & $0(1)$ & 1(1) & $8(1)$ \\
\hline $\mathrm{Cl}(1)$ & $29(1)$ & $43(1)$ & $13(1)$ & $-2(1)$ & $-1(1)$ & $16(1)$ \\
\hline $\mathrm{Cl}(2)$ & $92(1)$ & $72(1)$ & $81(2)$ & $23(1)$ & 11(1) & $46(1)$ \\
\hline $\mathrm{Cl}(3)$ & $72(1)$ & $86(1)$ & $72(1)$ & $-19(1)$ & $-5(1)$ & $40(1)$ \\
\hline $\mathrm{Cl}(4)$ & $85(1)$ & $64(1)$ & $50(1)$ & $-3(1)$ & $6(1)$ & $35(1)$ \\
\hline $\mathrm{P}(1)$ & $17(1)$ & $17(1)$ & 11(1) & 1(1) & 1(1) & $9(1)$ \\
\hline $\mathrm{P}(2)$ & $18(1)$ & $18(1)$ & $15(1)$ & 1(1) & $-1(1)$ & $8(1)$ \\
\hline $\mathrm{P}(3)$ & $20(1)$ & $18(1)$ & $14(1)$ & $-1(1)$ & $-2(1)$ & 11(1) \\
\hline $\mathrm{P}(4)$ & 19(1) & $17(1)$ & $18(1)$ & $-1(1)$ & $2(1)$ & $9(1)$ \\
\hline $\mathrm{C}(1)$ & $25(2)$ & $29(2)$ & $15(2)$ & $3(2)$ & 1(2) & $5(2)$ \\
\hline$C(2)$ & $19(2)$ & $22(2)$ & $19(2)$ & $6(2)$ & $0(2)$ & $6(2)$ \\
\hline $\mathrm{C}(3)$ & $14(2)$ & $21(2)$ & $13(2)$ & $-1(2)$ & $-1(2)$ & $3(2)$ \\
\hline$C(4)$ & $17(2)$ & $20(2)$ & $17(2)$ & $1(2)$ & $3(2)$ & $5(2)$ \\
\hline$C(5)$ & $20(2)$ & $21(2)$ & $23(2)$ & $-2(2)$ & $4(2)$ & $6(2)$ \\
\hline$C(6)$ & $23(2)$ & $24(2)$ & 22(3) & $-3(2)$ & $1(2)$ & $2(2)$ \\
\hline$C(7)$ & $25(2)$ & $23(2)$ & $20(2)$ & $4(2)$ & $0(2)$ & $10(2)$ \\
\hline $\mathrm{C}(8)$ & $44(3)$ & $36(2)$ & $33(3)$ & $9(2)$ & $0(2)$ & $24(2)$ \\
\hline $\mathrm{C}(9)$ & $34(2)$ & $34(2)$ & $54(4)$ & $16(2)$ & $3(2)$ & $17(2)$ \\
\hline $\mathrm{C}(10)$ & $44(3)$ & $55(3)$ & 28(3) & $8(2)$ & $-3(2)$ & $25(2)$ \\
\hline $\mathrm{C}(11)$ & $21(2)$ & $28(2)$ & 24(3) & $1(2)$ & $0(2)$ & $14(2)$ \\
\hline $\mathrm{C}(12)$ & $49(3)$ & $43(3)$ & $34(3)$ & $-2(2)$ & $-19(2)$ & $27(2)$ \\
\hline$C(13)$ & $31(2)$ & $37(2)$ & $38(3)$ & $13(2)$ & $6(2)$ & $19(2)$ \\
\hline$C(14)$ & $48(3)$ & $82(3)$ & $31(3)$ & $16(3)$ & $12(2)$ & $51(3)$ \\
\hline$C(15)$ & $36(2)$ & $40(2)$ & $17(2)$ & $-5(2)$ & $9(2)$ & $10(2)$ \\
\hline$C(16)$ & $75(5)$ & $98(5)$ & $33(5)$ & $-2(4)$ & 11(4) & $56(5)$ \\
\hline $\mathrm{C}(17)$ & $50(4)$ & $72(5)$ & $35(4)$ & $-24(4)$ & $-2(3)$ & $28(3)$ \\
\hline $\mathrm{C}(18)$ & $63(4)$ & $57(4)$ & $32(5)$ & $-2(3)$ & $23(4)$ & $10(3)$ \\
\hline C(19) & $24(2)$ & $18(2)$ & $21(2)$ & $1(2)$ & $1(2)$ & $11(2)$ \\
\hline$C(20)$ & $24(2)$ & $19(2)$ & $19(2)$ & $1(2)$ & $4(2)$ & $13(2)$ \\
\hline $\mathrm{C}(21)$ & $18(2)$ & $16(2)$ & $30(3)$ & $1(2)$ & $0(2)$ & $8(2)$ \\
\hline $\mathrm{C}(22)$ & $31(2)$ & $19(2)$ & $21(2)$ & $0(2)$ & $-4(2)$ & $16(2)$ \\
\hline
\end{tabular}




\begin{tabular}{|c|c|c|c|c|c|c|}
\hline$C(23)$ & $29(2)$ & $24(2)$ & $18(2)$ & $3(2)$ & $6(2)$ & $14(2)$ \\
\hline$C(24)$ & $26(2)$ & $18(2)$ & $20(2)$ & $-1(2)$ & $3(2)$ & $13(2)$ \\
\hline$C(25)$ & $23(2)$ & $23(2)$ & $21(2)$ & $-3(2)$ & $4(2)$ & $9(2)$ \\
\hline$C(26)$ & $24(2)$ & $50(3)$ & $38(3)$ & $-12(2)$ & $8(2)$ & $-2(2)$ \\
\hline $\mathrm{C}(27)$ & $35(2)$ & $30(2)$ & $39(3)$ & $-3(2)$ & $15(2)$ & $12(2)$ \\
\hline$C(28)$ & 43(3) & $56(3)$ & $36(3)$ & $22(2)$ & 19(2) & $28(2)$ \\
\hline C(29) & $36(2)$ & $36(2)$ & $20(3)$ & $0(2)$ & $-5(2)$ & $19(2)$ \\
\hline$C(30)$ & $35(3)$ & $56(3)$ & $32(3)$ & $-1(2)$ & $-8(2)$ & $14(2)$ \\
\hline $\mathrm{C}(31)$ & $57(3)$ & $33(2)$ & $32(3)$ & $-11(2)$ & $-11(2)$ & $19(2)$ \\
\hline$C(32)$ & $42(3)$ & $49(3)$ & 21(3) & $2(2)$ & $-7(2)$ & $23(2)$ \\
\hline$C(33)$ & $24(2)$ & $28(2)$ & $23(3)$ & $-6(2)$ & $4(2)$ & $14(2)$ \\
\hline$C(34)$ & $40(3)$ & $63(3)$ & $38(3)$ & $-19(2)$ & $-3(2)$ & $31(2)$ \\
\hline$C(35)$ & $27(2)$ & $36(2)$ & $38(3)$ & $2(2)$ & $0(2)$ & $17(2)$ \\
\hline$C(36)$ & $36(2)$ & $37(2)$ & $44(3)$ & $4(2)$ & $11(2)$ & $16(2)$ \\
\hline$C(37)$ & $22(2)$ & $22(2)$ & $23(2)$ & $3(2)$ & $1(2)$ & $13(2)$ \\
\hline C(38) & $25(2)$ & $32(2)$ & $20(2)$ & $4(2)$ & $-1(2)$ & $20(2)$ \\
\hline$C(39)$ & $25(2)$ & $34(2)$ & $17(2)$ & $-2(2)$ & $-3(2)$ & $17(2)$ \\
\hline $\mathrm{C}(40)$ & $19(2)$ & $24(2)$ & $19(2)$ & $-1(2)$ & $-2(2)$ & $12(2)$ \\
\hline $\mathrm{C}(41)$ & $19(2)$ & $24(2)$ & $20(2)$ & $4(2)$ & $2(2)$ & $14(2)$ \\
\hline $\mathrm{C}(42)$ & $22(2)$ & $22(2)$ & $19(2)$ & $1(2)$ & $-3(2)$ & $13(2)$ \\
\hline $\mathrm{C}(43)$ & $32(2)$ & $31(2)$ & $20(2)$ & $7(2)$ & $5(2)$ & $18(2)$ \\
\hline $\mathrm{C}(44)$ & $40(3)$ & $43(3)$ & $23(3)$ & $8(2)$ & $5(2)$ & $21(2)$ \\
\hline $\mathrm{C}(45)$ & $40(3)$ & $61(3)$ & $25(3)$ & $14(2)$ & $1(2)$ & $35(2)$ \\
\hline $\mathrm{C}(46)$ & $56(3)$ & $39(3)$ & $30(3)$ & $12(2)$ & $4(2)$ & $25(2)$ \\
\hline $\mathrm{C}(47)$ & $21(2)$ & $27(2)$ & $21(3)$ & $-2(2)$ & $-9(2)$ & $10(2)$ \\
\hline $\mathrm{C}(48)$ & $39(3)$ & $45(3)$ & $49(4)$ & $6(2)$ & $-18(2)$ & $7(2)$ \\
\hline C(49) & $42(3)$ & $34(2)$ & $42(3)$ & $-12(2)$ & $-2(2)$ & $17(2)$ \\
\hline$C(50)$ & $35(2)$ & $33(2)$ & $32(3)$ & $-2(2)$ & $1(2)$ & $13(2)$ \\
\hline $\mathrm{C}(51)$ & $28(2)$ & $22(2)$ & $20(2)$ & $-1(2)$ & $-6(2)$ & $16(2)$ \\
\hline$C(52)$ & $39(2)$ & $38(2)$ & $24(3)$ & $-10(2)$ & $-4(2)$ & $23(2)$ \\
\hline$C(53)$ & $34(2)$ & $28(2)$ & $23(3)$ & $-7(2)$ & $-8(2)$ & $16(2)$ \\
\hline C(54) & $53(3)$ & $22(2)$ & $30(3)$ & $-4(2)$ & $-11(2)$ & $20(2)$ \\
\hline$C(55)$ & $23(2)$ & $19(2)$ & $14(2)$ & $-1(2)$ & $1(2)$ & $12(2)$ \\
\hline$C(56)$ & $25(2)$ & $36(2)$ & $16(2)$ & $7(2)$ & $2(2)$ & $18(2)$ \\
\hline$C(57)$ & $34(2)$ & $36(2)$ & $28(3)$ & $13(2)$ & $12(2)$ & $17(2)$ \\
\hline C(58) & $23(2)$ & $36(2)$ & $27(3)$ & $5(2)$ & $9(2)$ & \\
\hline
\end{tabular}




\begin{tabular}{lllllll}
$\mathrm{C}(59)$ & $18(2)$ & $26(2)$ & $25(3)$ & $-3(2)$ & $2(2)$ & $12(2)$ \\
$\mathrm{C}(60)$ & $19(2)$ & $18(2)$ & $15(2)$ & $0(2)$ & $2(2)$ & $9(2)$ \\
$\mathrm{C}(61)$ & $20(2)$ & $21(2)$ & $18(2)$ & $-2(2)$ & $0(2)$ & $12(2)$ \\
$\mathrm{C}(62)$ & $20(2)$ & $20(2)$ & $16(2)$ & $-2(2)$ & $-5(2)$ & $12(2)$ \\
$\mathrm{C}(63)$ & $24(2)$ & $27(2)$ & $16(2)$ & $-2(2)$ & $0(2)$ & $10(2)$ \\
$\mathrm{C}(64)$ & $32(2)$ & $31(2)$ & $24(3)$ & $-6(2)$ & $0(2)$ & $18(2)$ \\
$\mathrm{C}(65)$ & $35(2)$ & $30(2)$ & $28(3)$ & $-15(2)$ & $-8(2)$ & $19(2)$ \\
$\mathrm{C}(66)$ & $22(2)$ & $24(2)$ & $23(2)$ & $-4(2)$ & $-6(2)$ & $10(2)$ \\
$\mathrm{C}(67)$ & $20(2)$ & $22(2)$ & $14(2)$ & $-3(2)$ & $-6(2)$ & $12(2)$ \\
$\mathrm{C}(68)$ & $17(2)$ & $19(2)$ & $19(2)$ & $1(2)$ & $-2(2)$ & $6(2)$ \\
$\mathrm{C}(69)$ & $16(2)$ & $21(2)$ & $15(2)$ & $4(2)$ & $0(2)$ & $9(2)$ \\
$\mathrm{C}(70)$ & $32(2)$ & $32(2)$ & $17(2)$ & $0(2)$ & $-1(2)$ & $21(2)$ \\
$\mathrm{C}(71)$ & $31(2)$ & $30(2)$ & $23(3)$ & $2(2)$ & $-4(2)$ & $13(2)$ \\
$\mathrm{C}(72)$ & $33(2)$ & $35(2)$ & $28(3)$ & $9(2)$ & $-1(2)$ & $23(2)$ \\
$\mathrm{C}(73)$ & $28(2)$ & $25(2)$ & $24(3)$ & $6(2)$ & $4(2)$ & $16(2)$ \\
$\mathrm{C}(74)$ & $19(2)$ & $20(2)$ & $18(2)$ & $2(2)$ & $2(2)$ & $11(2)$ \\
$\mathrm{C}(75)$ & $24(2)$ & $16(2)$ & $24(2)$ & $1(2)$ & $4(2)$ & $11(2)$ \\
$\mathrm{C}(81)$ & $85(4)$ & $65(3)$ & $23(3)$ & $4(3)$ & $4(3)$ & $37(3)$ \\
$\mathrm{C}(16 \mathrm{~A})$ & $67(7)$ & $46(4)$ & $21(7)$ & $-8(3)$ & $10(5)$ & $17(3)$ \\
$\mathrm{C}(17 \mathrm{~A})$ & $64(7)$ & $77(7)$ & $21(5)$ & $1(5)$ & $16(4)$ & $39(6)$ \\
$\mathrm{C}(18 \mathrm{~A})$ & $42(4)$ & $59(6)$ & $32(7)$ & $-11(5)$ & $13(3)$ & $16(3)$ \\
\hline
\end{tabular}


Table S10. Hydrogen coordinates ( x 104) and isotropic displacement parameters $\left(\AA^{2} \mathrm{x} 10^{3}\right)$ for cain07_sq.

\begin{tabular}{|c|c|c|c|c|}
\hline & $\mathrm{x}$ & $\mathrm{y}$ & $\mathrm{z}$ & $\mathrm{U}(\mathrm{eq})$ \\
\hline $\mathrm{H}(1)$ & 4793 & 4499 & 5390 & 32 \\
\hline $\mathrm{H}(5)$ & 5274 & 3734 & 5846 & 28 \\
\hline $\mathrm{H}(8 \mathrm{~A})$ & 3961 & 4990 & 6015 & 53 \\
\hline $\mathrm{H}(8 \mathrm{~B})$ & 4234 & 4836 & 6267 & 53 \\
\hline $\mathrm{H}(8 \mathrm{C})$ & 3760 & 4430 & 6091 & 53 \\
\hline $\mathrm{H}(9 \mathrm{~A})$ & 5020 & 5247 & 5606 & 61 \\
\hline $\mathrm{H}(9 \mathrm{~B})$ & 4990 & 5317 & 5978 & 61 \\
\hline $\mathrm{H}(9 \mathrm{C})$ & 4702 & 5469 & 5737 & 61 \\
\hline $\mathrm{H}(10 \mathrm{~A})$ & 4259 & 4660 & 5317 & 63 \\
\hline $\mathrm{H}(10 \mathrm{~B})$ & 3942 & 4852 & 5492 & 63 \\
\hline $\mathrm{H}(10 \mathrm{C})$ & 3787 & 4293 & 5512 & 63 \\
\hline $\mathrm{H}(12 \mathrm{~A})$ & 5304 & 3947 & 6860 & 60 \\
\hline $\mathrm{H}(12 \mathrm{~B})$ & 4996 & 4188 & 6741 & 60 \\
\hline $\mathrm{H}(12 \mathrm{C})$ & 5518 & 4358 & 6594 & 60 \\
\hline $\mathrm{H}(13 \mathrm{~A})$ & 4637 & 3156 & 6755 & 53 \\
\hline $\mathrm{H}(13 \mathrm{~B})$ & 4449 & 2982 & 6402 & 53 \\
\hline $\mathrm{H}(13 \mathrm{C})$ & 4289 & 3315 & 6589 & 53 \\
\hline $\mathrm{H}(14 \mathrm{~A})$ & 5450 & 3428 & 6544 & 68 \\
\hline $\mathrm{H}(14 \mathrm{~B})$ & 5666 & 3818 & 6265 & 68 \\
\hline $\mathrm{H}(14 \mathrm{C})$ & 5238 & 3281 & 6193 & 68 \\
\hline $\mathrm{H}(16 \mathrm{~A})$ & 5801 & 3896 & 5438 & 94 \\
\hline $\mathrm{H}(16 \mathrm{~B})$ & 5629 & 3662 & 5094 & 94 \\
\hline $\mathrm{H}(16 \mathrm{C})$ & 5301 & 3400 & 5397 & 94 \\
\hline $\mathrm{H}(17 \mathrm{~A})$ & 4635 & 3414 & 5100 & 80 \\
\hline $\mathrm{H}(17 \mathrm{~B})$ & 5001 & 3709 & 4820 & 80 \\
\hline $\mathrm{H}(17 \mathrm{C})$ & 4678 & 3907 & 4977 & 80 \\
\hline $\mathrm{H}(18 \mathrm{~A})$ & 5466 & 4663 & 5035 & 89 \\
\hline $\mathrm{H}(18 \mathrm{~B})$ & 5791 & 4429 & 4945 & 89 \\
\hline $\mathrm{H}(18 \mathrm{C})$ & 5852 & 4679 & 5283 & 89 \\
\hline $\mathrm{H}(21)$ & 1698 & 1330 & 5844 & 26 \\
\hline
\end{tabular}




\begin{tabular}{|c|c|c|c|}
\hline $\mathrm{H}(23)$ & 2923 & 1803 & 5355 \\
\hline $\mathrm{H}(26 \mathrm{~A})$ & 1257 & 1211 & 6550 \\
\hline $\mathrm{H}(26 \mathrm{~B})$ & 1404 & 970 & 6271 \\
\hline $\mathrm{H}(26 \mathrm{C})$ & 1312 & 1408 & 6196 \\
\hline $\mathrm{H}(27 \mathrm{~A})$ & 1810 & 2002 & 6747 \\
\hline $\mathrm{H}(27 \mathrm{~B})$ & 1827 & 2194 & 6396 \\
\hline $\mathrm{H}(27 \mathrm{C})$ & 2319 & 2341 & 6580 \\
\hline $\mathrm{H}(28 \mathrm{~A})$ & 1934 & 1328 & 6852 \\
\hline $\mathrm{H}(28 \mathrm{~B})$ & 2477 & 1624 & 6723 \\
\hline $\mathrm{H}(28 \mathrm{C})$ & 2110 & 1103 & 6580 \\
\hline $\mathrm{H}(30 \mathrm{~A})$ & 1365 & 1365 & 5360 \\
\hline $\mathrm{H}(30 \mathrm{~B})$ & 1307 & 853 & 5450 \\
\hline $\mathrm{H}(30 \mathrm{C})$ & 1259 & 974 & 5090 \\
\hline $\mathrm{H}(31 \mathrm{~A})$ & 2405 & 1028 & 5118 \\
\hline $\mathrm{H}(31 \mathrm{~B})$ & 1883 & 753 & 4957 \\
\hline $\mathrm{H}(31 \mathrm{C})$ & 1943 & 663 & 5322 \\
\hline $\mathrm{H}(32 \mathrm{~A})$ & 2101 & 1962 & 5041 \\
\hline $\mathrm{H}(32 \mathrm{~B})$ & 1961 & 1543 & 4786 \\
\hline $\mathrm{H}(32 \mathrm{C})$ & 2492 & 1816 & 4937 \\
\hline $\mathrm{H}(34 \mathrm{~A})$ & 3652 & 2207 & 5282 \\
\hline $\mathrm{H}(34 \mathrm{~B})$ & 3548 & 1721 & 5458 \\
\hline $\mathrm{H}(34 \mathrm{C})$ & 4079 & 2185 & 5464 \\
\hline $\mathrm{H}(35 \mathrm{~A})$ & 4139 & 2182 & 6018 \\
\hline $\mathrm{H}(35 \mathrm{~B})$ & 3627 & 1693 & 6022 \\
\hline $\mathrm{H}(35 \mathrm{C})$ & 3718 & 2136 & 6245 \\
\hline $\mathrm{H}(36 \mathrm{~A})$ & 3795 & 2920 & 5592 \\
\hline $\mathrm{H}(36 \mathrm{~B})$ & 4242 & 2897 & 5743 \\
\hline $\mathrm{H}(36 \mathrm{C})$ & 3851 & 2920 & 5968 \\
\hline $\mathrm{H}(37)$ & 2965 & 4946 & 5897 \\
\hline $\mathrm{H}(39)$ & 2238 & 3748 & 5380 \\
\hline $\mathrm{H}(44 \mathrm{~A})$ & 3009 & 4705 & 4833 \\
\hline $\mathrm{H}(44 \mathrm{~B})$ & 2672 & 4169 & 4965 \\
\hline $\mathrm{H}(44 \mathrm{C})$ & 3214 & 4488 & 5092 \\
\hline $\mathrm{H}(45 \mathrm{~A})$ & 2330 & 4844 & 4977 \\
\hline $\mathrm{H}(45 \mathrm{~B})$ & 2177 & 4827 & 5340 \\
\hline $\mathrm{H}(45 \mathrm{C})$ & 2024 & 4335 & 5154 \\
\hline
\end{tabular}




\begin{tabular}{|c|c|c|c|}
\hline $\mathrm{H}(46 \mathrm{~A})$ & 3199 & 5410 & 5136 \\
\hline $\mathrm{H}(46 \mathrm{~B})$ & 3435 & 5254 & 5412 \\
\hline $\mathrm{H}(46 \mathrm{C})$ & 3019 & 5378 & 5493 \\
\hline $\mathrm{H}(48 \mathrm{~A})$ & 1807 & 3018 & 5310 \\
\hline $\mathrm{H}(48 \mathrm{~B})$ & 1482 & 3177 & 5508 \\
\hline $\mathrm{H}(48 \mathrm{C})$ & 1349 & 2622 & 5504 \\
\hline $\mathrm{H}(49 \mathrm{~A})$ & 2378 & 2840 & 5586 \\
\hline $\mathrm{H}(49 \mathrm{~B})$ & 1905 & 2400 & 5740 \\
\hline $\mathrm{H}(49 \mathrm{C})$ & 2340 & 2769 & 5961 \\
\hline $\mathrm{H}(50 \mathrm{~A})$ & 1319 & 2519 & 6033 \\
\hline $\mathrm{H}(50 \mathrm{~B})$ & 1382 & 3047 & 6065 \\
\hline $\mathrm{H}(50 \mathrm{C})$ & 1716 & 2906 & 6268 \\
\hline $\mathrm{H}(52 \mathrm{~A})$ & 2511 & 4659 & 6865 \\
\hline $\mathrm{H}(52 \mathrm{~B})$ & 2267 & 4134 & 6708 \\
\hline $\mathrm{H}(52 \mathrm{C})$ & 2209 & 4553 & 6544 \\
\hline $\mathrm{H}(53 \mathrm{~A})$ & 3331 & 4779 & 6867 \\
\hline $\mathrm{H}(53 \mathrm{~B})$ & 3543 & 4658 & 6560 \\
\hline $\mathrm{H}(53 \mathrm{C})$ & 3101 & 4232 & 6748 \\
\hline $\mathrm{H}(54 \mathrm{~A})$ & 3195 & 5353 & 6622 \\
\hline $\mathrm{H}(54 \mathrm{~B})$ & 2896 & 5252 & 6300 \\
\hline $\mathrm{H}(54 \mathrm{C})$ & 3422 & 5305 & 6296 \\
\hline $\mathrm{H}(56)$ & 2901 & 2568 & 7372 \\
\hline $\mathrm{H}(57)$ & 2160 & 2109 & 7623 \\
\hline $\mathrm{H}(58)$ & 1522 & 2244 & 7505 \\
\hline $\mathrm{H}(59)$ & 1617 & 2799 & 7119 \\
\hline $\mathrm{H}(61)$ & 2092 & 3425 & 6747 \\
\hline $\mathrm{H}(63)$ & 2988 & 3656 & 7417 \\
\hline $\mathrm{H}(64)$ & 3300 & 4372 & 7707 \\
\hline $\mathrm{H}(65)$ & 4050 & 5035 & 7563 \\
\hline $\mathrm{H}(66)$ & 4476 & 4978 & 7140 \\
\hline $\mathrm{H}(68)$ & 4613 & 4530 & 6744 \\
\hline $\mathrm{H}(70)$ & 4000 & 3588 & 7394 \\
\hline $\mathrm{H}(71)$ & 4439 & 3273 & 7636 \\
\hline $\mathrm{H}(72)$ & 4338 & 2529 & 7464 \\
\hline $\mathrm{H}(73)$ & 3810 & 2118 & 7054 \\
\hline $\mathrm{H}(75)$ & 3197 & 2002 & 6689 \\
\hline
\end{tabular}




\begin{tabular}{lllll}
$\mathrm{H}(81)$ & 3301 & 3377 & 5209 & 69 \\
$\mathrm{H}(16 \mathrm{D})$ & 5159 & 3354 & 5309 & 75 \\
$\mathrm{H}(16 \mathrm{E})$ & 5063 & 3461 & 4955 & 75 \\
$\mathrm{H}(16 \mathrm{~F})$ & 4668 & 3348 & 5225 & 75 \\
$\mathrm{H}(17 \mathrm{D})$ & 4718 & 4043 & 4939 & 79 \\
$\mathrm{H}(17 \mathrm{E})$ & 5223 & 4221 & 4764 & 79 \\
$\mathrm{H}(17 \mathrm{~F})$ & 5165 & 4571 & 5009 & 79 \\
$\mathrm{H}(18 \mathrm{D})$ & 5896 & 4622 & 5251 & 72 \\
$\mathrm{H}(18 \mathrm{E})$ & 5899 & 4174 & 5083 & 72 \\
$\mathrm{H}(18 \mathrm{~F})$ & 5882 & 4198 & 5460 & 72 \\
\hline
\end{tabular}




\section{Data for 2b}

Table S11. Crystal data and structure refinement for cain30_a_sq (2b).

Identification code

Empirical formula

Formula weight

Temperature

Wavelength

Crystal system

Space group

Unit cell dimensions

Volume

Z

Density (calculated)

Absorption coefficient

$\mathrm{F}(000)$

Crystal size

Theta range for data collection

Index ranges

Reflections collected

Independent reflections

Completeness to theta $=26.000^{\circ}$

Absorption correction

Max. and min. transmission

Refinement method

Data / restraints / parameters

Goodness-of-fit on $\mathrm{F}^{2}$

Final $\mathrm{R}$ indices [I $>2 \operatorname{sigma}(\mathrm{I})]$

$\mathrm{R}$ indices (all data)

Extinction coefficient

Largest diff. peak and hole

SQUEEZE cain30_a_sq

C76 H100 Cl4 Ir P4

1471.43

$100.0 \mathrm{~K}$

$0.71073 \AA$

Trigonal

P-3

$\mathrm{a}=18.6016(7) \AA \quad \alpha=90^{\circ}$.

$\mathrm{b}=18.6016(7) \AA \quad \beta=90^{\circ}$.

$\mathrm{c}=14.2012(6) \AA \quad \gamma=120^{\circ}$.

4255.6(4) $\AA^{3}$

2

$1.148 \mathrm{Mg} / \mathrm{m}^{3}$

$1.804 \mathrm{~mm}^{-1}$

1522

$0.12 \times 0.12 \times 0.04 \mathrm{~mm}^{3}$

1.912 to $30.115^{\circ}$.

$-24<=\mathrm{h}<=25,-25<=\mathrm{k}<=25,-19<=1<=19$

78720

$7770[\mathrm{R}($ int $)=0.0478]$

$99.9 \%$

Semi-empirical from equivalents

0.0991 and 0.0678

Full-matrix least-squares on $\mathrm{F}^{2}$

7770 / 0 / 309

1.028

$\mathrm{R} 1=0.0327, \mathrm{wR} 2=0.0799$

$\mathrm{R} 1=0.0437, \mathrm{wR} 2=0.0847$

$\mathrm{n} / \mathrm{a}$

1.134 and -1.188 e. $\AA^{-3}$

found $413 \mathrm{e} / \mathrm{uc}$;calc'd for $9 \mathrm{CHCl}_{3}: 522 \mathrm{e} / \mathrm{uc}$ 
Table S12. Atomic coordinates ( $\left.\times 10^{4}\right)$ and equivalent isotropic displacement parameters $\left(\AA^{2} \times 10^{3}\right)$ for cain30_a_sq. U(eq) is defined as one third of the trace of the orthogonalized $U^{i j}$ tensor.

\begin{tabular}{|c|c|c|c|c|}
\hline & $\mathrm{x}$ & $\mathrm{y}$ & $\mathrm{z}$ & $\mathrm{U}(\mathrm{eq})$ \\
\hline $\operatorname{Ir}(1)$ & 6667 & 3333 & $5822(1)$ & $24(1)$ \\
\hline $\mathrm{Cl}(1)$ & 6667 & 3333 & $7528(1)$ & $59(1)$ \\
\hline $\mathrm{P}(1)$ & 6667 & 3333 & $4247(1)$ & $23(1)$ \\
\hline $\mathrm{P}(2)$ & $5758(1)$ & 1954(1) & 5761(1) & $28(1)$ \\
\hline $\mathrm{C}(1)$ & $6752(2)$ & $2490(2)$ & $3705(2)$ & $27(1)$ \\
\hline$C(2)$ & $7297(2)$ & $2665(2)$ & $2955(2)$ & $35(1)$ \\
\hline$C(3)$ & $7357(2)$ & $2044(2)$ & 2491(2) & $46(1)$ \\
\hline $\mathrm{C}(4)$ & $6854(2)$ & $1225(2)$ & $2767(2)$ & $46(1)$ \\
\hline$C(5)$ & $6315(2)$ & $1041(2)$ & $3505(2)$ & $37(1)$ \\
\hline$C(6)$ & $6250(2)$ & $1657(2)$ & $4015(2)$ & $29(1)$ \\
\hline $\mathrm{C}(7)$ & $5686(2)$ & 1381(2) & $4814(2)$ & $31(1)$ \\
\hline $\mathrm{C}(8)$ & $4958(2)$ & $1326(2)$ & $6647(2)$ & $30(1)$ \\
\hline $\mathrm{C}(9)$ & $4148(2)$ & $1246(2)$ & $6612(2)$ & $33(1)$ \\
\hline $\mathrm{C}(10)$ & $3692(2)$ & $1053(2)$ & $7444(2)$ & $38(1)$ \\
\hline $\mathrm{C}(11)$ & $3967(2)$ & $916(2)$ & $8291(2)$ & $42(1)$ \\
\hline $\mathrm{C}(12)$ & $4697(2)$ & $892(2)$ & $8274(2)$ & $40(1)$ \\
\hline $\mathrm{C}(13)$ & $5201(2)$ & $1072(2)$ & $7481(2)$ & $33(1)$ \\
\hline $\mathrm{C}(14)$ & $3689(2)$ & $1276(2)$ & $5707(2)$ & $40(1)$ \\
\hline$C(15)$ & $4159(2)$ & $2065(2)$ & $5129(3)$ & $68(1)$ \\
\hline$C(16)$ & $3493(2)$ & $527(2)$ & $5103(3)$ & $61(1)$ \\
\hline $\mathrm{C}(17)$ & 2844(2) & $1207(3)$ & 5921(3) & $70(1)$ \\
\hline$C(18)$ & $3472(2)$ & $771(2)$ & $9209(2)$ & $53(1)$ \\
\hline$C(22)$ & $5936(2)$ & $907(2)$ & $7587(2)$ & $38(1)$ \\
\hline $\mathrm{C}(19)$ & $4023(3)$ & $1365(3)$ & 9971(3) & $68(1)$ \\
\hline$C(20)$ & $3066(4)$ & $-104(3)$ & 9489(3) & $71(2)$ \\
\hline $\mathrm{C}(21)$ & $2750(4)$ & 1001(4) & $9078(4)$ & $77(2)$ \\
\hline$C(23)$ & $5846(4)$ & $397(4)$ & $8484(4)$ & $50(2)$ \\
\hline$C(24)$ & $5893(3)$ & $326(3)$ & $6780(4)$ & $36(1)$ \\
\hline$C(25)$ & $6762(3)$ & $1674(3)$ & $7620(4)$ & $42(1)$ \\
\hline$C\left(19^{\prime}\right)$ & $3890(13)$ & $468(15)$ & $10043(13)$ & $77(2)$ \\
\hline $\mathrm{C}\left(20^{\prime}\right)$ & $2548(12)$ & $31(12)$ & $8996(12)$ & $68(1)$ \\
\hline
\end{tabular}




\begin{tabular}{llllr}
$\mathrm{C}\left(21^{\prime}\right)$ & $3505(14)$ & $1481(10)$ & $9477(13)$ & $71(2)$ \\
$\mathrm{C}\left(23^{\prime}\right)$ & $6484(5)$ & $1380(5)$ & $8471(5)$ & $58(2)$ \\
$\mathrm{C}\left(24^{\prime}\right)$ & $6596(4)$ & $1182(5)$ & $6798(5)$ & $49(2)$ \\
$\mathrm{C}\left(25^{\prime}\right)$ & $5598(5)$ & $-16(4)$ & $7716(7)$ & $60(2)$ \\
$\mathrm{Cl}(2)$ & $6384(1)$ & $2339(1)$ & $10441(2)$ & $134(1)$ \\
$\mathrm{C}(26)$ & 6667 & 3333 & $10038(5)$ & $90(3)$ \\
\hline
\end{tabular}


Table S13. Bond lengths $[\AA ̊ \mathrm{~A}]$ and angles $\left[{ }^{\circ}\right]$ for cain30_a_sq.

\begin{tabular}{|c|c|}
\hline $\operatorname{Ir}(1)-\mathrm{Cl}(1)$ & $2.4227(12)$ \\
\hline $\operatorname{Ir}(1)-\mathrm{P}(1)$ & $2.2369(10)$ \\
\hline $\operatorname{Ir}(1)-\mathrm{P}(2) \# 1$ & $2.2614(6)$ \\
\hline $\operatorname{Ir}(1)-\mathrm{P}(2)$ & $2.2613(6)$ \\
\hline $\operatorname{Ir}(1)-P(2) \# 2$ & $2.2612(6)$ \\
\hline $\mathrm{P}(1)-\mathrm{C}(1)$ & $1.825(2)$ \\
\hline $\mathrm{P}(1)-\mathrm{C}(1) \# 1$ & $1.825(2)$ \\
\hline $\mathrm{P}(1)-\mathrm{C}(1) \# 2$ & $1.825(2)$ \\
\hline$P(2)-C(7)$ & $1.679(3)$ \\
\hline$P(2)-C(8)$ & $1.850(3)$ \\
\hline $\mathrm{C}(1)-\mathrm{C}(2)$ & $1.392(3)$ \\
\hline$C(1)-C(6)$ & $1.420(3)$ \\
\hline $\mathrm{C}(2)-\mathrm{H}(2)$ & 0.9500 \\
\hline$C(2)-C(3)$ & $1.382(4)$ \\
\hline $\mathrm{C}(3)-\mathrm{H}(3)$ & 0.9500 \\
\hline$C(3)-C(4)$ & $1.386(4)$ \\
\hline $\mathrm{C}(4)-\mathrm{H}(4)$ & 0.9500 \\
\hline$C(4)-C(5)$ & $1.371(4)$ \\
\hline $\mathrm{C}(5)-\mathrm{H}(5)$ & 0.9500 \\
\hline$C(5)-C(6)$ & $1.411(4)$ \\
\hline$C(6)-C(7)$ & $1.454(4)$ \\
\hline $\mathrm{C}(8)-\mathrm{C}(9)$ & $1.440(4)$ \\
\hline $\mathrm{C}(8)-\mathrm{C}(13)$ & $1.429(3)$ \\
\hline $\mathrm{C}(9)-\mathrm{C}(10)$ & $1.393(4)$ \\
\hline $\mathrm{C}(9)-\mathrm{C}(14)$ & $1.560(4)$ \\
\hline $\mathrm{C}(10)-\mathrm{H}(10)$ & 0.9500 \\
\hline$C(10)-C(11)$ & $1.379(4)$ \\
\hline $\mathrm{C}(11)-\mathrm{C}(12)$ & $1.380(4)$ \\
\hline $\mathrm{C}(11)-\mathrm{C}(18)$ & $1.541(4)$ \\
\hline $\mathrm{C}(12)-\mathrm{H}(12)$ & 0.9500 \\
\hline$C(12)-C(13)$ & $1.395(4)$ \\
\hline$C(13)-C(22)$ & $1.552(4)$ \\
\hline$C(14)-C(15)$ & $1.519(4)$ \\
\hline$C(14)-C(16)$ & $1.518(5)$ \\
\hline
\end{tabular}




\begin{tabular}{|c|c|}
\hline $\mathrm{C}(14)-\mathrm{C}(17)$ & $1.542(4)$ \\
\hline $\mathrm{C}(15)-\mathrm{H}(15 \mathrm{~A})$ & 0.9800 \\
\hline $\mathrm{C}(15)-\mathrm{H}(15 \mathrm{~B})$ & 0.9800 \\
\hline $\mathrm{C}(15)-\mathrm{H}(15 \mathrm{C})$ & 0.9800 \\
\hline $\mathrm{C}(16)-\mathrm{H}(16 \mathrm{~A})$ & 0.9800 \\
\hline $\mathrm{C}(16)-\mathrm{H}(16 \mathrm{~B})$ & 0.9800 \\
\hline $\mathrm{C}(16)-\mathrm{H}(16 \mathrm{C})$ & 0.9800 \\
\hline $\mathrm{C}(17)-\mathrm{H}(17 \mathrm{~A})$ & 0.9800 \\
\hline $\mathrm{C}(17)-\mathrm{H}(17 \mathrm{~B})$ & 0.9800 \\
\hline $\mathrm{C}(17)-\mathrm{H}(17 \mathrm{C})$ & 0.9800 \\
\hline$C(18)-C(19)$ & $1.520(6)$ \\
\hline$C(18)-C(20)$ & $1.466(6)$ \\
\hline$C(18)-C(21)$ & $1.610(7)$ \\
\hline $\mathrm{C}(18)-\mathrm{C}\left(19^{\prime}\right)$ & $1.66(2)$ \\
\hline $\mathrm{C}(18)-\mathrm{C}\left(20^{\prime}\right)$ & $1.604(18)$ \\
\hline $\mathrm{C}(18)-\mathrm{C}\left(21^{\prime}\right)$ & $1.345(17)$ \\
\hline$C(22)-C(23)$ & $1.547(6)$ \\
\hline$C(22)-C(24)$ & $1.549(6)$ \\
\hline$C(22)-C(25)$ & $1.485(6)$ \\
\hline $\mathrm{C}(22)-\mathrm{C}\left(23^{\prime}\right)$ & $1.579(7)$ \\
\hline$C(22)-C\left(24^{\prime}\right)$ & $1.548(8)$ \\
\hline $\mathrm{C}(22)-\mathrm{C}\left(25^{\prime}\right)$ & $1.516(8)$ \\
\hline $\mathrm{C}(19)-\mathrm{H}(19 \mathrm{~A})$ & 0.9800 \\
\hline $\mathrm{C}(19)-\mathrm{H}(19 \mathrm{~B})$ & 0.9800 \\
\hline $\mathrm{C}(19)-\mathrm{H}(19 \mathrm{C})$ & 0.9800 \\
\hline $\mathrm{C}(20)-\mathrm{H}(20 \mathrm{~A})$ & 0.9800 \\
\hline $\mathrm{C}(20)-\mathrm{H}(20 \mathrm{~B})$ & 0.9800 \\
\hline $\mathrm{C}(20)-\mathrm{H}(20 \mathrm{C})$ & 0.9800 \\
\hline $\mathrm{C}(21)-\mathrm{H}(21 \mathrm{~A})$ & 0.9800 \\
\hline $\mathrm{C}(21)-\mathrm{H}(21 \mathrm{~B})$ & 0.9800 \\
\hline $\mathrm{C}(21)-\mathrm{H}(21 \mathrm{C})$ & 0.9800 \\
\hline $\mathrm{C}(23)-\mathrm{H}(23 \mathrm{~A})$ & 0.9800 \\
\hline $\mathrm{C}(23)-\mathrm{H}(23 \mathrm{~B})$ & 0.9800 \\
\hline $\mathrm{C}(23)-\mathrm{H}(23 \mathrm{C})$ & 0.9800 \\
\hline $\mathrm{C}(24)-\mathrm{H}(24 \mathrm{~A})$ & 0.9800 \\
\hline $\mathrm{C}(24)-\mathrm{H}(24 \mathrm{~B})$ & 0.9800 \\
\hline
\end{tabular}




\begin{tabular}{|c|c|}
\hline $\mathrm{C}(24)-\mathrm{H}(24 \mathrm{C})$ & 0.9800 \\
\hline $\mathrm{C}(25)-\mathrm{H}(25 \mathrm{~A})$ & 0.9800 \\
\hline $\mathrm{C}(25)-\mathrm{H}(25 \mathrm{~B})$ & 0.9800 \\
\hline $\mathrm{C}(25)-\mathrm{H}(25 \mathrm{C})$ & 0.9800 \\
\hline $\mathrm{C}\left(19^{\prime}\right)-\mathrm{H}(19 \mathrm{D})$ & 0.9800 \\
\hline $\mathrm{C}\left(1^{\prime}\right)-\mathrm{H}(19 \mathrm{E})$ & 0.9800 \\
\hline $\mathrm{C}\left(19^{\prime}\right)-\mathrm{H}(19 \mathrm{~F})$ & 0.9800 \\
\hline $\mathrm{C}\left(20^{\prime}\right)-\mathrm{H}(20 \mathrm{D})$ & 0.9800 \\
\hline $\mathrm{C}\left(20^{\prime}\right)-\mathrm{H}(20 \mathrm{E})$ & 0.9800 \\
\hline $\mathrm{C}\left(20^{\prime}\right)-\mathrm{H}(20 \mathrm{~F})$ & 0.9800 \\
\hline $\mathrm{C}\left(21^{\prime}\right)-\mathrm{H}(21 \mathrm{D})$ & 0.9800 \\
\hline $\mathrm{C}\left(21^{\prime}\right)-\mathrm{H}(21 \mathrm{E})$ & 0.9800 \\
\hline $\mathrm{C}\left(21^{\prime}\right)-\mathrm{H}(21 \mathrm{~F})$ & 0.9800 \\
\hline $\mathrm{C}\left(23^{\prime}\right)-\mathrm{H}(23 \mathrm{D})$ & 0.9800 \\
\hline $\mathrm{C}\left(23^{\prime}\right)-\mathrm{H}(23 \mathrm{E})$ & 0.9800 \\
\hline $\mathrm{C}\left(23^{\prime}\right)-\mathrm{H}(23 \mathrm{~F})$ & 0.9800 \\
\hline $\mathrm{C}\left(24^{\prime}\right)-\mathrm{H}(24 \mathrm{D})$ & 0.9800 \\
\hline $\mathrm{C}\left(24^{\prime}\right)-\mathrm{H}(24 \mathrm{E})$ & 0.9800 \\
\hline $\mathrm{C}\left(24^{\prime}\right)-\mathrm{H}(24 \mathrm{~F})$ & 0.9800 \\
\hline $\mathrm{C}\left(25^{\prime}\right)-\mathrm{H}(25 \mathrm{D})$ & 0.9800 \\
\hline $\mathrm{C}\left(25^{\prime}\right)-\mathrm{H}(25 \mathrm{E})$ & 0.9800 \\
\hline $\mathrm{C}\left(25^{\prime}\right)-\mathrm{H}(25 \mathrm{~F})$ & 0.9800 \\
\hline $\mathrm{Cl}(2)-\mathrm{C}(26)$ & $1.747(3)$ \\
\hline $\mathrm{C}(26)-\mathrm{Cl}(2) \# 2$ & $1.747(3)$ \\
\hline $\mathrm{C}(26)-\mathrm{Cl}(2) \# 1$ & $1.747(3)$ \\
\hline $\mathrm{C}(26)-\mathrm{H}(26)$ & 1.0000 \\
\hline $\mathrm{P}(1)-\operatorname{Ir}(1)-\mathrm{Cl}(1)$ & 180.0 \\
\hline $\mathrm{P}(1)-\operatorname{Ir}(1)-\mathrm{P}(2) \# 1$ & $87.793(16)$ \\
\hline $\mathrm{P}(1)-\operatorname{Ir}(1)-\mathrm{P}(2) \# 2$ & $87.793(16)$ \\
\hline $\mathrm{P}(1)-\operatorname{Ir}(1)-\mathrm{P}(2)$ & $87.792(16)$ \\
\hline $\mathrm{P}(2)-\operatorname{Ir}(1)-\mathrm{Cl}(1)$ & $92.208(16)$ \\
\hline $\mathrm{P}(2) \# 2-\operatorname{Ir}(1)-\mathrm{Cl}(1)$ & $92.207(16)$ \\
\hline $\mathrm{P}(2) \# 1-\operatorname{Ir}(1)-\mathrm{Cl}(1)$ & $92.207(16)$ \\
\hline $\mathrm{P}(2)-\operatorname{Ir}(1)-\mathrm{P}(2) \# 2$ & $119.853(2)$ \\
\hline $\mathrm{P}(2)-\operatorname{Ir}(1)-\mathrm{P}(2) \# 1$ & $119.852(2)$ \\
\hline
\end{tabular}




\begin{tabular}{|c|c|}
\hline $\mathrm{P}(2) \# 1-\operatorname{Ir}(1)-\mathrm{P}(2) \# 2$ & $119.854(3)$ \\
\hline $\mathrm{C}(1) \# 2-\mathrm{P}(1)-\operatorname{Ir}(1)$ & $114.95(8)$ \\
\hline C(1)-P(1)-Ir(1) & $114.95(8)$ \\
\hline C(1)\#1-P(1)-Ir(1) & $114.95(8)$ \\
\hline $\mathrm{C}(1)-\mathrm{P}(1)-\mathrm{C}(1) \# 1$ & $103.48(9)$ \\
\hline $\mathrm{C}(1)-\mathrm{P}(1)-\mathrm{C}(1) \# 2$ & $103.48(9)$ \\
\hline $\mathrm{C}(1) \# 1-\mathrm{P}(1)-\mathrm{C}(1) \# 2$ & $103.48(9)$ \\
\hline $\mathrm{C}(7)-\mathrm{P}(2)-\operatorname{Ir}(1)$ & $121.89(9)$ \\
\hline $\mathrm{C}(7)-\mathrm{P}(2)-\mathrm{C}(8)$ & $111.11(12)$ \\
\hline $\mathrm{C}(8)-\mathrm{P}(2)-\operatorname{Ir}(1)$ & $126.81(8)$ \\
\hline $\mathrm{C}(2)-\mathrm{C}(1)-\mathrm{P}(1)$ & 119.17(19) \\
\hline$C(2)-C(1)-C(6)$ & $119.9(2)$ \\
\hline $\mathrm{C}(6)-\mathrm{C}(1)-\mathrm{P}(1)$ & $120.93(18)$ \\
\hline $\mathrm{C}(1)-\mathrm{C}(2)-\mathrm{H}(2)$ & 119.3 \\
\hline $\mathrm{C}(3)-\mathrm{C}(2)-\mathrm{C}(1)$ & $121.5(3)$ \\
\hline $\mathrm{C}(3)-\mathrm{C}(2)-\mathrm{H}(2)$ & 119.3 \\
\hline $\mathrm{C}(2)-\mathrm{C}(3)-\mathrm{H}(3)$ & 120.3 \\
\hline $\mathrm{C}(2)-\mathrm{C}(3)-\mathrm{C}(4)$ & $119.4(3)$ \\
\hline $\mathrm{C}(4)-\mathrm{C}(3)-\mathrm{H}(3)$ & 120.3 \\
\hline $\mathrm{C}(3)-\mathrm{C}(4)-\mathrm{H}(4)$ & 120.1 \\
\hline $\mathrm{C}(5)-\mathrm{C}(4)-\mathrm{C}(3)$ & $119.8(3)$ \\
\hline $\mathrm{C}(5)-\mathrm{C}(4)-\mathrm{H}(4)$ & 120.1 \\
\hline $\mathrm{C}(4)-\mathrm{C}(5)-\mathrm{H}(5)$ & 118.7 \\
\hline$C(4)-C(5)-C(6)$ & $122.6(3)$ \\
\hline $\mathrm{C}(6)-\mathrm{C}(5)-\mathrm{H}(5)$ & 118.7 \\
\hline$C(1)-C(6)-C(7)$ & $126.3(2)$ \\
\hline$C(5)-C(6)-C(1)$ & $116.7(2)$ \\
\hline$C(5)-C(6)-C(7)$ & $117.0(2)$ \\
\hline $\mathrm{C}(6)-\mathrm{C}(7)-\mathrm{P}(2)$ & $125.89(19)$ \\
\hline $\mathrm{C}(9)-\mathrm{C}(8)-\mathrm{P}(2)$ & $120.77(18)$ \\
\hline $\mathrm{C}(13)-\mathrm{C}(8)-\mathrm{P}(2)$ & $119.04(19)$ \\
\hline $\mathrm{C}(13)-\mathrm{C}(8)-\mathrm{C}(9)$ & $118.8(2)$ \\
\hline C(8)-C(9)-C(14) & $126.0(2)$ \\
\hline $\mathrm{C}(10)-\mathrm{C}(9)-\mathrm{C}(8)$ & $117.9(2)$ \\
\hline$C(10)-C(9)-C(14)$ & $115.8(2)$ \\
\hline $\mathrm{C}(9)-\mathrm{C}(10)-\mathrm{H}(10)$ & 118.2 \\
\hline
\end{tabular}




\begin{tabular}{|c|c|}
\hline$C(11)-C(10)-C(9)$ & $123.7(3)$ \\
\hline $\mathrm{C}(11)-\mathrm{C}(10)-\mathrm{H}(10)$ & 118.2 \\
\hline $\mathrm{C}(10)-\mathrm{C}(11)-\mathrm{C}(12)$ & $116.9(3)$ \\
\hline$C(10)-C(11)-C(18)$ & $121.7(3)$ \\
\hline $\mathrm{C}(12)-\mathrm{C}(11)-\mathrm{C}(18)$ & $121.4(3)$ \\
\hline $\mathrm{C}(11)-\mathrm{C}(12)-\mathrm{H}(12)$ & 118.0 \\
\hline$C(11)-C(12)-C(13)$ & $124.1(3)$ \\
\hline $\mathrm{C}(13)-\mathrm{C}(12)-\mathrm{H}(12)$ & 118.0 \\
\hline $\mathrm{C}(8)-\mathrm{C}(13)-\mathrm{C}(22)$ & $126.8(2)$ \\
\hline $\mathrm{C}(12)-\mathrm{C}(13)-\mathrm{C}(8)$ & $117.6(3)$ \\
\hline $\mathrm{C}(12)-\mathrm{C}(13)-\mathrm{C}(22)$ & $115.3(2)$ \\
\hline $\mathrm{C}(15)-\mathrm{C}(14)-\mathrm{C}(9)$ & $114.8(2)$ \\
\hline$C(15)-C(14)-C(17)$ & $104.9(3)$ \\
\hline $\mathrm{C}(16)-\mathrm{C}(14)-\mathrm{C}(9)$ & $108.5(2)$ \\
\hline$C(16)-C(14)-C(15)$ & $109.9(3)$ \\
\hline$C(16)-C(14)-C(17)$ & $105.6(3)$ \\
\hline $\mathrm{C}(17)-\mathrm{C}(14)-\mathrm{C}(9)$ & $112.8(3)$ \\
\hline $\mathrm{C}(14)-\mathrm{C}(15)-\mathrm{H}(15 \mathrm{~A})$ & 109.5 \\
\hline $\mathrm{C}(14)-\mathrm{C}(15)-\mathrm{H}(15 \mathrm{~B})$ & 109.5 \\
\hline $\mathrm{C}(14)-\mathrm{C}(15)-\mathrm{H}(15 \mathrm{C})$ & 109.5 \\
\hline $\mathrm{H}(15 \mathrm{~A})-\mathrm{C}(15)-\mathrm{H}(15 \mathrm{~B})$ & 109.5 \\
\hline $\mathrm{H}(15 \mathrm{~A})-\mathrm{C}(15)-\mathrm{H}(15 \mathrm{C})$ & 109.5 \\
\hline $\mathrm{H}(15 \mathrm{~B})-\mathrm{C}(15)-\mathrm{H}(15 \mathrm{C})$ & 109.5 \\
\hline $\mathrm{C}(14)-\mathrm{C}(16)-\mathrm{H}(16 \mathrm{~A})$ & 109.5 \\
\hline $\mathrm{C}(14)-\mathrm{C}(16)-\mathrm{H}(16 \mathrm{~B})$ & 109.5 \\
\hline $\mathrm{C}(14)-\mathrm{C}(16)-\mathrm{H}(16 \mathrm{C})$ & 109.5 \\
\hline $\mathrm{H}(16 \mathrm{~A})-\mathrm{C}(16)-\mathrm{H}(16 \mathrm{~B})$ & 109.5 \\
\hline $\mathrm{H}(16 \mathrm{~A})-\mathrm{C}(16)-\mathrm{H}(16 \mathrm{C})$ & 109.5 \\
\hline $\mathrm{H}(16 \mathrm{~B})-\mathrm{C}(16)-\mathrm{H}(16 \mathrm{C})$ & 109.5 \\
\hline $\mathrm{C}(14)-\mathrm{C}(17)-\mathrm{H}(17 \mathrm{~A})$ & 109.5 \\
\hline $\mathrm{C}(14)-\mathrm{C}(17)-\mathrm{H}(17 \mathrm{~B})$ & 109.5 \\
\hline $\mathrm{C}(14)-\mathrm{C}(17)-\mathrm{H}(17 \mathrm{C})$ & 109.5 \\
\hline $\mathrm{H}(17 \mathrm{~A})-\mathrm{C}(17)-\mathrm{H}(17 \mathrm{~B})$ & 109.5 \\
\hline $\mathrm{H}(17 \mathrm{~A})-\mathrm{C}(17)-\mathrm{H}(17 \mathrm{C})$ & 109.5 \\
\hline $\mathrm{H}(17 \mathrm{~B})-\mathrm{C}(17)-\mathrm{H}(17 \mathrm{C})$ & 109.5 \\
\hline $\mathrm{C}(11)-\mathrm{C}(18)-\mathrm{C}(21)$ & $111.1(3)$ \\
\hline
\end{tabular}




\begin{tabular}{|c|c|}
\hline $\mathrm{C}(11)-\mathrm{C}(18)-\mathrm{C}\left(19^{\prime}\right)$ & $108.8(6)$ \\
\hline $\mathrm{C}(11)-\mathrm{C}(18)-\mathrm{C}\left(20^{\prime}\right)$ & $106.3(6)$ \\
\hline$C(19)-C(18)-C(11)$ & $110.3(3)$ \\
\hline $\mathrm{C}(19)-\mathrm{C}(18)-\mathrm{C}(21)$ & $103.8(4)$ \\
\hline $\mathrm{C}(20)-\mathrm{C}(18)-\mathrm{C}(11)$ & $110.8(3)$ \\
\hline $\mathrm{C}(20)-\mathrm{C}(18)-\mathrm{C}(19)$ & $113.6(4)$ \\
\hline$C(20)-C(18)-C(21)$ & $106.9(4)$ \\
\hline $\mathrm{C}\left(20^{\prime}\right)-\mathrm{C}(18)-\mathrm{C}\left(19^{\prime}\right)$ & $109.0(11)$ \\
\hline $\mathrm{C}\left(21^{\prime}\right)-\mathrm{C}(18)-\mathrm{C}(11)$ & 109.7(7) \\
\hline $\mathrm{C}\left(21^{\prime}\right)-\mathrm{C}(18)-\mathrm{C}\left(19^{\prime}\right)$ & $109.4(13)$ \\
\hline $\mathrm{C}\left(21^{\prime}\right)-\mathrm{C}(18)-\mathrm{C}\left(20^{\prime}\right)$ & $113.6(13)$ \\
\hline $\mathrm{C}(13)-\mathrm{C}(22)-\mathrm{C}\left(23^{\prime}\right)$ & $110.7(4)$ \\
\hline$C(23)-C(22)-C(13)$ & $111.4(3)$ \\
\hline$C(23)-C(22)-C(24)$ & $103.3(3)$ \\
\hline $\mathrm{C}(24)-\mathrm{C}(22)-\mathrm{C}(13)$ & $108.9(3)$ \\
\hline$C(25)-C(22)-C(13)$ & $113.8(3)$ \\
\hline$C(25)-C(22)-C(23)$ & $107.8(4)$ \\
\hline$C(25)-C(22)-C(24)$ & $111.2(4)$ \\
\hline $\mathrm{C}\left(24^{\prime}\right)-\mathrm{C}(22)-\mathrm{C}(13)$ & $119.8(3)$ \\
\hline $\mathrm{C}\left(24^{\prime}\right)-\mathrm{C}(22)-\mathrm{C}\left(23^{\prime}\right)$ & $102.0(5)$ \\
\hline $\mathrm{C}\left(25^{\prime}\right)-\mathrm{C}(22)-\mathrm{C}(13)$ & 109.1(4) \\
\hline $\mathrm{C}\left(25^{\prime}\right)-\mathrm{C}(22)-\mathrm{C}\left(23^{\prime}\right)$ & $108.8(4)$ \\
\hline $\mathrm{C}\left(25^{\prime}\right)-\mathrm{C}(22)-\mathrm{C}\left(24^{\prime}\right)$ & $105.9(5)$ \\
\hline $\mathrm{C}(18)-\mathrm{C}(19)-\mathrm{H}(19 \mathrm{~A})$ & 109.5 \\
\hline $\mathrm{C}(18)-\mathrm{C}(19)-\mathrm{H}(19 \mathrm{~B})$ & 109.5 \\
\hline $\mathrm{C}(18)-\mathrm{C}(19)-\mathrm{H}(19 \mathrm{C})$ & 109.5 \\
\hline $\mathrm{H}(19 \mathrm{~A})-\mathrm{C}(19)-\mathrm{H}(19 \mathrm{~B})$ & 109.5 \\
\hline $\mathrm{H}(19 \mathrm{~A})-\mathrm{C}(19)-\mathrm{H}(19 \mathrm{C})$ & 109.5 \\
\hline $\mathrm{H}(19 \mathrm{~B})-\mathrm{C}(19)-\mathrm{H}(19 \mathrm{C})$ & 109.5 \\
\hline $\mathrm{C}(18)-\mathrm{C}(20)-\mathrm{H}(20 \mathrm{~A})$ & 109.5 \\
\hline $\mathrm{C}(18)-\mathrm{C}(20)-\mathrm{H}(20 \mathrm{~B})$ & 109.5 \\
\hline $\mathrm{C}(18)-\mathrm{C}(20)-\mathrm{H}(20 \mathrm{C})$ & 109.5 \\
\hline $\mathrm{H}(20 \mathrm{~A})-\mathrm{C}(20)-\mathrm{H}(20 \mathrm{~B})$ & 109.5 \\
\hline$H(20 A)-C(20)-H(20 C)$ & 109.5 \\
\hline $\mathrm{H}(20 \mathrm{~B})-\mathrm{C}(20)-\mathrm{H}(20 \mathrm{C})$ & 109.5 \\
\hline $\mathrm{C}(18)-\mathrm{C}(21)-\mathrm{H}(21 \mathrm{~A})$ & 109.5 \\
\hline
\end{tabular}




\begin{tabular}{|c|c|}
\hline $\mathrm{C}(18)-\mathrm{C}(21)-\mathrm{H}(21 \mathrm{~B})$ & 109.5 \\
\hline $\mathrm{C}(18)-\mathrm{C}(21)-\mathrm{H}(21 \mathrm{C})$ & 109.5 \\
\hline $\mathrm{H}(21 \mathrm{~A})-\mathrm{C}(21)-\mathrm{H}(21 \mathrm{~B})$ & 109.5 \\
\hline $\mathrm{H}(21 \mathrm{~A})-\mathrm{C}(21)-\mathrm{H}(21 \mathrm{C})$ & 109.5 \\
\hline $\mathrm{H}(21 \mathrm{~B})-\mathrm{C}(21)-\mathrm{H}(21 \mathrm{C})$ & 109.5 \\
\hline $\mathrm{C}(22)-\mathrm{C}(23)-\mathrm{H}(23 \mathrm{~A})$ & 109.5 \\
\hline $\mathrm{C}(22)-\mathrm{C}(23)-\mathrm{H}(23 \mathrm{~B})$ & 109.5 \\
\hline $\mathrm{C}(22)-\mathrm{C}(23)-\mathrm{H}(23 \mathrm{C})$ & 109.5 \\
\hline $\mathrm{H}(23 \mathrm{~A})-\mathrm{C}(23)-\mathrm{H}(23 \mathrm{~B})$ & 109.5 \\
\hline $\mathrm{H}(23 \mathrm{~A})-\mathrm{C}(23)-\mathrm{H}(23 \mathrm{C})$ & 109.5 \\
\hline $\mathrm{H}(23 \mathrm{~B})-\mathrm{C}(23)-\mathrm{H}(23 \mathrm{C})$ & 109.5 \\
\hline $\mathrm{C}(22)-\mathrm{C}(24)-\mathrm{H}(24 \mathrm{~A})$ & 109.5 \\
\hline $\mathrm{C}(22)-\mathrm{C}(24)-\mathrm{H}(24 \mathrm{~B})$ & 109.5 \\
\hline $\mathrm{C}(22)-\mathrm{C}(24)-\mathrm{H}(24 \mathrm{C})$ & 109.5 \\
\hline $\mathrm{H}(24 \mathrm{~A})-\mathrm{C}(24)-\mathrm{H}(24 \mathrm{~B})$ & 109.5 \\
\hline $\mathrm{H}(24 \mathrm{~A})-\mathrm{C}(24)-\mathrm{H}(24 \mathrm{C})$ & 109.5 \\
\hline $\mathrm{H}(24 \mathrm{~B})-\mathrm{C}(24)-\mathrm{H}(24 \mathrm{C})$ & 109.5 \\
\hline $\mathrm{C}(22)-\mathrm{C}(25)-\mathrm{H}(25 \mathrm{~A})$ & 109.5 \\
\hline $\mathrm{C}(22)-\mathrm{C}(25)-\mathrm{H}(25 \mathrm{~B})$ & 109.5 \\
\hline $\mathrm{C}(22)-\mathrm{C}(25)-\mathrm{H}(25 \mathrm{C})$ & 109.5 \\
\hline $\mathrm{H}(25 \mathrm{~A})-\mathrm{C}(25)-\mathrm{H}(25 \mathrm{~B})$ & 109.5 \\
\hline $\mathrm{H}(25 \mathrm{~A})-\mathrm{C}(25)-\mathrm{H}(25 \mathrm{C})$ & 109.5 \\
\hline $\mathrm{H}(25 \mathrm{~B})-\mathrm{C}(25)-\mathrm{H}(25 \mathrm{C})$ & 109.5 \\
\hline $\mathrm{C}(18)-\mathrm{C}\left(19^{\prime}\right)-\mathrm{H}(19 \mathrm{D})$ & 109.5 \\
\hline $\mathrm{C}(18)-\mathrm{C}\left(19^{\prime}\right)-\mathrm{H}(19 \mathrm{E})$ & 109.5 \\
\hline $\mathrm{C}(18)-\mathrm{C}\left(1^{\prime}\right)-\mathrm{H}(19 \mathrm{~F})$ & 109.5 \\
\hline $\mathrm{H}(19 \mathrm{D})-\mathrm{C}\left(19^{\prime}\right)-\mathrm{H}(19 \mathrm{E})$ & 109.5 \\
\hline $\mathrm{H}(19 \mathrm{D})-\mathrm{C}\left(19^{\prime}\right)-\mathrm{H}(19 \mathrm{~F})$ & 109.5 \\
\hline $\mathrm{H}(19 \mathrm{E})-\mathrm{C}\left(19^{\prime}\right)-\mathrm{H}(19 \mathrm{~F})$ & 109.5 \\
\hline $\mathrm{C}(18)-\mathrm{C}\left(20^{\prime}\right)-\mathrm{H}(20 \mathrm{D})$ & 109.5 \\
\hline $\mathrm{C}(18)-\mathrm{C}\left(20^{\prime}\right)-\mathrm{H}(20 \mathrm{E})$ & 109.5 \\
\hline $\mathrm{C}(18)-\mathrm{C}\left(20^{\prime}\right)-\mathrm{H}(20 \mathrm{~F})$ & 109.5 \\
\hline $\mathrm{H}(20 \mathrm{D})-\mathrm{C}\left(20^{\prime}\right)-\mathrm{H}(20 \mathrm{E})$ & 109.5 \\
\hline $\mathrm{H}(20 \mathrm{D})-\mathrm{C}\left(20^{\prime}\right)-\mathrm{H}(20 \mathrm{~F})$ & 109.5 \\
\hline $\mathrm{H}(20 \mathrm{E})-\mathrm{C}\left(20^{\prime}\right)-\mathrm{H}(20 \mathrm{~F})$ & 109.5 \\
\hline $\mathrm{C}(18)-\mathrm{C}\left(21^{\prime}\right)-\mathrm{H}(21 \mathrm{D})$ & 109. \\
\hline
\end{tabular}




\begin{tabular}{|c|c|}
\hline $\mathrm{C}(18)-\mathrm{C}\left(21^{\prime}\right)-\mathrm{H}(21 \mathrm{E})$ & 109.5 \\
\hline $\mathrm{C}(18)-\mathrm{C}\left(21^{\prime}\right)-\mathrm{H}(21 \mathrm{~F})$ & 109.5 \\
\hline $\mathrm{H}(21 \mathrm{D})-\mathrm{C}\left(21^{\prime}\right)-\mathrm{H}(21 \mathrm{E})$ & 109.5 \\
\hline $\mathrm{H}(21 \mathrm{D})-\mathrm{C}\left(21^{\prime}\right)-\mathrm{H}(21 \mathrm{~F})$ & 109.5 \\
\hline $\mathrm{H}(21 \mathrm{E})-\mathrm{C}\left(21^{\prime}\right)-\mathrm{H}(21 \mathrm{~F})$ & 109.5 \\
\hline $\mathrm{C}(22)-\mathrm{C}\left(23^{\prime}\right)-\mathrm{H}(23 \mathrm{D})$ & 109.5 \\
\hline $\mathrm{C}(22)-\mathrm{C}\left(23^{\prime}\right)-\mathrm{H}(23 \mathrm{E})$ & 109.5 \\
\hline $\mathrm{C}(22)-\mathrm{C}\left(23^{\prime}\right)-\mathrm{H}(23 \mathrm{~F})$ & 109.5 \\
\hline $\mathrm{H}(23 \mathrm{D})-\mathrm{C}\left(23^{\prime}\right)-\mathrm{H}(23 \mathrm{E})$ & 109.5 \\
\hline $\mathrm{H}(23 \mathrm{D})-\mathrm{C}\left(23^{\prime}\right)-\mathrm{H}(23 \mathrm{~F})$ & 109.5 \\
\hline $\mathrm{H}(23 \mathrm{E})-\mathrm{C}\left(23^{\prime}\right)-\mathrm{H}(23 \mathrm{~F})$ & 109.5 \\
\hline $\mathrm{C}(22)-\mathrm{C}\left(24^{\prime}\right)-\mathrm{H}(24 \mathrm{D})$ & 109.5 \\
\hline $\mathrm{C}(22)-\mathrm{C}\left(24^{\prime}\right)-\mathrm{H}(24 \mathrm{E})$ & 109.5 \\
\hline $\mathrm{C}(22)-\mathrm{C}\left(24^{\prime}\right)-\mathrm{H}(24 \mathrm{~F})$ & 109.5 \\
\hline $\mathrm{H}(24 \mathrm{D})-\mathrm{C}\left(24^{\prime}\right)-\mathrm{H}(24 \mathrm{E})$ & 109.5 \\
\hline $\mathrm{H}(24 \mathrm{D})-\mathrm{C}\left(24^{\prime}\right)-\mathrm{H}(24 \mathrm{~F})$ & 109.5 \\
\hline $\mathrm{H}(24 \mathrm{E})-\mathrm{C}\left(24^{\prime}\right)-\mathrm{H}(24 \mathrm{~F})$ & 109.5 \\
\hline $\mathrm{C}(22)-\mathrm{C}\left(25^{\prime}\right)-\mathrm{H}(25 \mathrm{D})$ & 109.5 \\
\hline $\mathrm{C}(22)-\mathrm{C}\left(25^{\prime}\right)-\mathrm{H}(25 \mathrm{E})$ & 109.5 \\
\hline $\mathrm{C}(22)-\mathrm{C}\left(25^{\prime}\right)-\mathrm{H}(25 \mathrm{~F})$ & 109.5 \\
\hline $\mathrm{H}(25 \mathrm{D})-\mathrm{C}\left(25^{\prime}\right)-\mathrm{H}(25 \mathrm{E})$ & 109.5 \\
\hline $\mathrm{H}(25 \mathrm{D})-\mathrm{C}\left(25^{\prime}\right)-\mathrm{H}(25 \mathrm{~F})$ & 109.5 \\
\hline $\mathrm{H}(25 \mathrm{E})-\mathrm{C}\left(25^{\prime}\right)-\mathrm{H}(25 \mathrm{~F})$ & 109.5 \\
\hline $\mathrm{Cl}(2)-\mathrm{C}(26)-\mathrm{Cl}(2) \# 1$ & $109.8(2)$ \\
\hline $\mathrm{Cl}(2)-\mathrm{C}(26)-\mathrm{Cl}(2) \# 2$ & $109.8(2)$ \\
\hline $\mathrm{Cl}(2) \# 1-\mathrm{C}(26)-\mathrm{Cl}(2) \# 2$ & $109.8(2)$ \\
\hline $\mathrm{Cl}(2)-\mathrm{C}(26)-\mathrm{H}(26)$ & 109.1 \\
\hline $\mathrm{Cl}(2) \# 2-\mathrm{C}(26)-\mathrm{H}(26)$ & 109.1 \\
\hline $\mathrm{Cl}(2) \# 1-\mathrm{C}(26)-\mathrm{H}(26)$ & 109.1 \\
\hline
\end{tabular}

Symmetry transformations used to generate equivalent atoms:

$\# 1-y+1, x-y, z \quad \# 2-x+y+1,-x+1, z$ 
Table S14. Anisotropic displacement parameters $\left(\AA^{2} \times 10^{3}\right)$ for cain30_a_sq. The anisotropic displacement factor exponent takes the form: $-2 \pi^{2}\left[h^{2} a^{* 2} U^{11}+\ldots+2 h k a^{*} b^{*} U^{12}\right]$

\begin{tabular}{|c|c|c|c|c|c|c|}
\hline & $\mathrm{U}^{11}$ & $\mathrm{U}^{22}$ & $\mathrm{U}^{33}$ & $U^{23}$ & $\mathrm{U}^{13}$ & $\mathrm{U}^{12}$ \\
\hline $\operatorname{Ir}(1)$ & $26(1)$ & $26(1)$ & 21(1) & 0 & 0 & $13(1)$ \\
\hline $\mathrm{Cl}(1)$ & $78(1)$ & $78(1)$ & $22(1)$ & 0 & 0 & $39(1)$ \\
\hline $\mathrm{P}(1)$ & $24(1)$ & $24(1)$ & $20(1)$ & 0 & 0 & $12(1)$ \\
\hline $\mathrm{P}(2)$ & $27(1)$ & $25(1)$ & $28(1)$ & $3(1)$ & $0(1)$ & 11(1) \\
\hline$C(1)$ & $29(1)$ & $28(1)$ & $25(1)$ & $-4(1)$ & $-4(1)$ & $14(1)$ \\
\hline $\mathrm{C}(2)$ & $39(1)$ & $34(1)$ & $30(1)$ & $-4(1)$ & $3(1)$ & 18(1) \\
\hline$C(3)$ & $54(2)$ & $48(2)$ & $38(2)$ & $-10(1)$ & $9(1)$ & $26(2)$ \\
\hline $\mathrm{C}(4)$ & $58(2)$ & $40(2)$ & $44(2)$ & $-15(1)$ & $-2(1)$ & $28(2)$ \\
\hline$C(5)$ & $41(2)$ & $30(1)$ & $40(1)$ & $-7(1)$ & $-7(1)$ & $18(1)$ \\
\hline$C(6)$ & $31(1)$ & $29(1)$ & 28(1) & $-4(1)$ & $-7(1)$ & $16(1)$ \\
\hline$C(7)$ & $30(1)$ & $28(1)$ & $33(1)$ & $0(1)$ & $-3(1)$ & $14(1)$ \\
\hline $\mathrm{C}(8)$ & $30(1)$ & $25(1)$ & 29(1) & $3(1)$ & $1(1)$ & $10(1)$ \\
\hline $\mathrm{C}(9)$ & $31(1)$ & $28(1)$ & $35(1)$ & $5(1)$ & $2(1)$ & $12(1)$ \\
\hline$C(10)$ & $36(1)$ & $30(1)$ & $43(2)$ & $3(1)$ & $8(1)$ & $12(1)$ \\
\hline $\mathrm{C}(11)$ & $45(2)$ & $33(1)$ & $35(1)$ & $0(1)$ & $8(1)$ & $10(1)$ \\
\hline $\mathrm{C}(12)$ & $48(2)$ & $31(1)$ & $30(1)$ & $2(1)$ & $-2(1)$ & $13(1)$ \\
\hline$C(13)$ & $37(1)$ & $26(1)$ & $30(1)$ & $2(1)$ & $-2(1)$ & 11(1) \\
\hline$C(14)$ & $28(1)$ & $43(2)$ & $45(2)$ & $16(1)$ & $4(1)$ & $15(1)$ \\
\hline$C(15)$ & $36(2)$ & $69(2)$ & 91(3) & $50(2)$ & $4(2)$ & $21(2)$ \\
\hline$C(16)$ & $55(2)$ & $68(2)$ & $55(2)$ & $-5(2)$ & $-22(2)$ & $27(2)$ \\
\hline $\mathrm{C}(17)$ & $42(2)$ & $104(3)$ & $67(2)$ & $29(2)$ & $11(2)$ & $40(2)$ \\
\hline$C(18)$ & $58(2)$ & $51(2)$ & $36(2)$ & 1(1) & $14(1)$ & $18(2)$ \\
\hline$C(22)$ & $43(2)$ & $36(1)$ & $34(1)$ & $4(1)$ & $-6(1)$ & $20(1)$ \\
\hline C(19) & $77(3)$ & $66(3)$ & $42(2)$ & $-13(2)$ & $9(2)$ & 21(3) \\
\hline $\mathrm{C}(20)$ & $96(4)$ & $42(2)$ & $51(3)$ & $4(2)$ & $42(3)$ & $16(2)$ \\
\hline $\mathrm{C}(21)$ & 79(4) & $110(5)$ & $54(3)$ & $6(3)$ & $22(3)$ & $56(4)$ \\
\hline $\mathrm{C}(23)$ & $63(4)$ & $55(4)$ & $42(3)$ & $12(3)$ & $2(3)$ & $38(3)$ \\
\hline $\mathrm{C}(24)$ & 41(3) & $31(2)$ & $38(3)$ & $0(2)$ & $-7(2)$ & $20(2)$ \\
\hline$C(25)$ & 41(3) & $40(3)$ & $46(3)$ & $-1(2)$ & $-12(2)$ & $21(2)$ \\
\hline $\mathrm{C}\left(19^{\prime}\right)$ & 79(4) & $110(5)$ & $54(3)$ & $6(3)$ & $22(3)$ & $56(4)$ \\
\hline $\mathrm{C}\left(20^{\prime}\right)$ & $77(3)$ & $66(3)$ & $42(2)$ & $-13(2)$ & $9(2)$ & $21(3)$ \\
\hline
\end{tabular}




\begin{tabular}{lllllll}
$\mathrm{C}\left(21^{\prime}\right)$ & $96(4)$ & $42(2)$ & $51(3)$ & $4(2)$ & $42(3)$ & $16(2)$ \\
$\mathrm{C}\left(23^{\prime}\right)$ & $59(5)$ & $50(4)$ & $52(4)$ & $6(3)$ & $-19(4)$ & $17(4)$ \\
$\mathrm{C}\left(24^{\prime}\right)$ & $45(4)$ & $52(4)$ & $53(4)$ & $6(3)$ & $-6(3)$ & $27(3)$ \\
$\mathrm{C}\left(25^{\prime}\right)$ & $51(4)$ & $42(4)$ & $82(6)$ & $8(4)$ & $-17(4)$ & $19(3)$ \\
$\mathrm{Cl}(2)$ & $123(1)$ & $109(1)$ & $171(2)$ & $2(1)$ & $-42(1)$ & $59(1)$ \\
$\mathrm{C}(26)$ & $121(4)$ & $121(4)$ & $27(3)$ & 0 & 0 & $61(2)$ \\
\hline
\end{tabular}


Table S15. Hydrogen coordinates ( x 104) and isotropic displacement parameters $\left(\AA^{2} \mathrm{x} 10^{3}\right)$ for cain30_a_sq.

\begin{tabular}{|c|c|c|c|c|}
\hline & $\mathrm{x}$ & $\mathrm{y}$ & $\mathrm{z}$ & $\mathrm{U}(\mathrm{eq})$ \\
\hline $\mathrm{H}(2)$ & 7636 & 3224 & 2759 & 42 \\
\hline $\mathrm{H}(3)$ & 7739 & 2176 & 1987 & 55 \\
\hline $\mathrm{H}(4)$ & 6883 & 793 & 2445 & 55 \\
\hline $\mathrm{H}(5)$ & 5971 & 477 & 3680 & 45 \\
\hline $\mathrm{H}(10)$ & 3163 & 1013 & 7428 & 46 \\
\hline $\mathrm{H}(12)$ & 4866 & 744 & 8838 & 48 \\
\hline $\mathrm{H}(15 \mathrm{~A})$ & 4281 & 2546 & 5522 & 101 \\
\hline $\mathrm{H}(15 \mathrm{~B})$ & 3819 & 2043 & 4590 & 101 \\
\hline $\mathrm{H}(15 \mathrm{C})$ & 4680 & 2116 & 4903 & 101 \\
\hline $\mathrm{H}(16 \mathrm{~A})$ & 4011 & 543 & 4929 & 91 \\
\hline $\mathrm{H}(16 \mathrm{~B})$ & 3202 & 535 & 4532 & 91 \\
\hline $\mathrm{H}(16 \mathrm{C})$ & 3140 & 18 & 5459 & 91 \\
\hline $\mathrm{H}(17 \mathrm{~A})$ & 2487 & 684 & 6249 & 104 \\
\hline $\mathrm{H}(17 \mathrm{~B})$ & 2579 & 1221 & 5330 & 104 \\
\hline $\mathrm{H}(17 \mathrm{C})$ & 2929 & 1673 & 6320 & 104 \\
\hline $\mathrm{H}(19 \mathrm{~A})$ & 4466 & 1246 & 10126 & 102 \\
\hline $\mathrm{H}(19 \mathrm{~B})$ & 3690 & 1296 & 10534 & 102 \\
\hline $\mathrm{H}(19 \mathrm{C})$ & 4268 & 1938 & 9743 & 102 \\
\hline $\mathrm{H}(20 \mathrm{~A})$ & 2699 & -450 & 8981 & 107 \\
\hline $\mathrm{H}(20 \mathrm{~B})$ & 2738 & -187 & 10061 & 107 \\
\hline $\mathrm{H}(20 \mathrm{C})$ & 3488 & -260 & 9612 & 107 \\
\hline $\mathrm{H}(21 \mathrm{~A})$ & 2984 & 1553 & 8794 & 115 \\
\hline $\mathrm{H}(21 \mathrm{~B})$ & 2511 & 999 & 9694 & 115 \\
\hline $\mathrm{H}(21 \mathrm{C})$ & 2316 & 589 & 8667 & 115 \\
\hline $\mathrm{H}(23 \mathrm{~A})$ & 5932 & 740 & 9042 & 75 \\
\hline $\mathrm{H}(23 \mathrm{~B})$ & 6261 & 219 & 8474 & 75 \\
\hline $\mathrm{H}(23 \mathrm{C})$ & 5289 & -92 & 8504 & 75 \\
\hline $\mathrm{H}(24 \mathrm{~A})$ & 5348 & -181 & 6786 & 55 \\
\hline $\mathrm{H}(24 \mathrm{~B})$ & 6328 & 182 & 6869 & 55 \\
\hline $\mathrm{H}(24 \mathrm{C})$ & 5975 & 610 & 6175 & 55 \\
\hline
\end{tabular}




\begin{tabular}{|c|c|c|c|c|}
\hline $\mathrm{H}(25 \mathrm{~A})$ & 6853 & 1988 & 7035 & 63 \\
\hline $\mathrm{H}(25 \mathrm{~B})$ & 7195 & 1525 & 7690 & 63 \\
\hline $\mathrm{H}(25 \mathrm{C})$ & 6782 & 2015 & 8156 & 63 \\
\hline $\mathrm{H}(19 \mathrm{D})$ & 3906 & -25 & 9829 & 115 \\
\hline $\mathrm{H}(19 \mathrm{E})$ & 3555 & 334 & 10618 & 115 \\
\hline $\mathrm{H}(19 \mathrm{~F})$ & 4456 & 916 & 10175 & 115 \\
\hline $\mathrm{H}(20 \mathrm{D})$ & 2352 & 143 & 8403 & 102 \\
\hline $\mathrm{H}(20 \mathrm{E})$ & 2179 & -8 & 9510 & 102 \\
\hline $\mathrm{H}(20 \mathrm{~F})$ & 2550 & -493 & 8943 & 102 \\
\hline $\mathrm{H}(21 \mathrm{D})$ & 4077 & 1890 & 9649 & 107 \\
\hline $\mathrm{H}(21 \mathrm{E})$ & 3141 & 1373 & 10020 & 107 \\
\hline $\mathrm{H}(21 \mathrm{~F})$ & 3323 & 1697 & 8955 & 107 \\
\hline $\mathrm{H}(23 \mathrm{D})$ & 6751 & 1980 & 8366 & 87 \\
\hline $\mathrm{H}(23 \mathrm{E})$ & 6910 & 1224 & 8563 & 87 \\
\hline $\mathrm{H}(23 \mathrm{~F})$ & 6131 & 1230 & 9031 & 87 \\
\hline $\mathrm{H}(24 \mathrm{D})$ & 6331 & 889 & 6213 & 74 \\
\hline $\mathrm{H}(24 \mathrm{E})$ & 7024 & 1049 & 6982 & 74 \\
\hline $\mathrm{H}(24 \mathrm{~F})$ & 6852 & 1782 & 6698 & 74 \\
\hline $\mathrm{H}(25 \mathrm{D})$ & 5223 & -213 & 8260 & 90 \\
\hline $\mathrm{H}(25 \mathrm{E})$ & 6059 & -120 & 7823 & 90 \\
\hline $\mathrm{H}(25 \mathrm{~F})$ & 5293 & -313 & 7149 & 90 \\
\hline $\mathrm{H}(26)$ & 6667 & 3333 & 9333 & 108 \\
\hline
\end{tabular}

